\title{
Z/m-GRADED LIE ALGEBRAS AND PERVERSE SHEAVES, I
}

\author{
GEORGE LUSZTIG AND ZHIWEI YUN
}

ABSTRACT. We give a block decomposition of the equivariant derived category arising from a cyclically graded Lie algebra. This generalizes certain aspects of the generalized Springer correspondence to the graded setting.

\section{Contents}

Introduction

1. Recollections on $\mathbf{Z}$-graded Lie algebras

2. $\quad \mathbf{Z} \mapsto$-gradings and $\epsilon$-spirals

3. Admissible systems

4. Spiral induction

5. Study of a pair of spirals

6. Spiral restriction

7. The categories $\mathcal{Q}\left(\mathfrak{g}_{\delta}\right), \mathcal{Q}^{\prime}\left(\mathfrak{g}_{\delta}\right)$

8. Monomial and quasi-monomial objects

9. Examples

References

\section{INTRODUCTION}

0.1 . Let $\mathbf{k}$ be an algebraically closed field of characteristic $p \geq 0$. We fix an integer $m>0$ such that $m<p$ whenever $p>0$ and we write $\mathbf{Z} / m$ instead of $\mathbf{Z} / m \mathbf{Z}$. For $n \in \mathbf{Z}$, let $\underline{n}$ denote the image of $n$ in $\mathbf{Z} / m$.

We also fix $G$, a semisimple simply connected algebraic group over $\mathbf{k}$ and a $\mathbf{Z} / m$ grading $\mathfrak{g}=\oplus_{i \in \mathbf{Z} / m} \mathfrak{g}_{i}$ (see 0.11) for the Lie algebra $\mathfrak{g}$ of $G$; we shall assume that either $p=0$ or that $p$ is so large relative to $G$, that we can operate with $\mathfrak{g}$ as if $p$ was 0 .

For any integer $d$ invertible in $\mathbf{k}$ let $\mu_{d}=\left\{z \in \mathbf{k}^{*} ; z^{d}=1\right\}$. The $\mathbf{Z} / m$-grading on $\mathfrak{g}$ is the same as an action of $\mu_{m}$ on $G$ or a homomorphism $\tilde{\vartheta}: \mu_{m} \rightarrow \operatorname{Aut}(G)$. $\left(\tilde{\vartheta}\right.$ induces a homomorphism $\tilde{\theta}: \mu_{m} \rightarrow \operatorname{Aut}(\mathfrak{g})$ and for $i \in \mathbf{Z} / m$ we have $\mathfrak{g}_{i}=\{x \in$ $\left.\mathfrak{g} ; \tilde{\theta}(z) x=z^{i} x \quad \forall z \in \mu_{m}\right\}$.) Let $G_{\underline{0}}=\left\{g \in G ; g \tilde{\vartheta}(z)=\tilde{\vartheta}(z) g \quad \forall z \in \mu_{m}\right\}$, be a connected reductive subgroup of $G$ with Lie algebra $\mathfrak{g}_{0}$. For any $i \in \mathbf{Z} / m$, the Ad-action of $G_{\underline{0}}$ on $\mathfrak{g}$ leaves stable $\mathfrak{g}_{i}$ and its closed subset $\mathfrak{g}_{i}^{\text {nil }}:=\mathfrak{g}_{i} \cap \mathfrak{g}^{\text {nil }}$. (Here $\mathfrak{g}^{\text {nil }}$ is the variety of nilpotent elements in $\mathfrak{g}$.)

Received by the editors October 12, 2016, and, in revised form, June 23, 2017.

2010 Mathematics Subject Classification. Primary 20G99.

The first author was supported by NSF grant DMS-1566618.

The second author was supported by NSF grant DMS-1302071 and the Packard Foundation. 
We are interested in studying the equivariant derived categories (see 0.11) $\mathcal{D}_{G_{\underline{0}}}\left(\mathfrak{g}_{i}\right), \mathcal{D}_{G_{\underline{0}}}\left(\mathfrak{g}_{i}^{\text {nil }}\right)$. More specifically, we would like to classify $G_{\underline{0}}$-equivariant simple perverse sheaves with support in $\mathfrak{g}_{i}^{\text {nil }}$ and (in the case where $p>0$ ) their Fourier-Deligne transform. The simple perverse sheaves in $\mathcal{D}_{G_{0}}\left(\mathfrak{g}_{i}^{\text {nil }}\right)$ are in bijection with the pair $(\mathcal{O}, \mathcal{L})$, where $\mathcal{O}$ is a nilpotent $G_{0^{-}}$orbit in $\mathfrak{g}_{i}$ and $\mathcal{L}$ is (the isomorphism class of) an irreducible $G_{\underline{0}}$-equivariant local system on $\mathcal{O}$. (The pair $(\mathcal{O}, \mathcal{L})$ gives rise to the simple perverse sheaf $P$ with support equal to the closure of $\mathcal{O}$ and with $\left.P\right|_{\mathcal{O}}=\mathcal{L}[\operatorname{dim} \mathcal{O}]$.) We denote the set of such $(\mathcal{O}, \mathcal{L})$ by $\mathcal{I}\left(\mathfrak{g}_{i}\right)$. This is a finite set, since the $G_{\underline{0}}$-action on $\mathfrak{g}_{i}^{\text {nil }}$ has only finitely many orbits. Alternatively, if we choose $e \in \mathcal{O}$, then the local system $\mathcal{L}$ corresponds to an irreducible representation of $\pi_{0}\left(G_{\underline{0}}(e)\right)$ (see 0.11 ), where $G_{\underline{0}}(e)$ is the stabilizer of $e$ under $G_{\underline{0}}$.

There are many $\mathbf{Z} / m$-graded Lie algebras which appear in nature.

0.2. In this subsection we assume that $m=2$ and $\mathbf{k}=\mathbf{C}$. Then the $\mathbf{Z} / 2$-grading $\mathfrak{g}=\mathfrak{k} \oplus \mathfrak{p}$ (with $\mathfrak{k}=\mathfrak{g}_{\underline{0}}, \mathfrak{p}=\mathfrak{g}_{\underline{1}}$ ) has been extensively studied in connection with the theory of symmetric spaces and the representation theory of real semisimple groups. In particular, the nilpotent $G_{\underline{0}}$-orbits on $\mathfrak{p}$ are known to be in bijection with the nilpotent orbits in the Lie algebra of a real form of $G$ determined by the Z/2-grading (Kostant and Sekiguchi).

0.3. Another class of examples comes from cyclic quivers. In this subsection we assume that $m \geq 2$. We consider the simplest such example where $V$ is a $\mathbf{k}$-vector space equipped with a $\mathbf{Z} / m$-grading $V=\oplus_{i \in \mathbf{Z} / m} V_{i}$ (see 0.11 ) and $G=S L(V)$ with the $\mathbf{Z} / m$-grading given by

$$
\mathfrak{g}_{i}=\left\{T \in \mathfrak{g}=\mathfrak{s l}(V) ; T\left(V_{j}\right) \subset V_{j+i} \quad \forall j \in \mathbf{Z} / m\right\} .
$$

In this case we have $G_{\underline{0}}=S\left(\prod_{i \in \mathbf{Z} / m} G L\left(V_{i}\right)\right)$, the intersection of $S L(V)$ with the Levi subgroup $\prod_{i} G L\left(V_{i}\right)$ of a parabolic subgroup of $G L(V)$. The subspace $\mathfrak{g}_{1}$ is

$$
\oplus_{i \in \mathbf{Z} / m} \operatorname{Hom}\left(V_{i}, V_{i+1}\right) .
$$

We may consider a quiver $Q$ with $m$ vertices indexed by $\mathbf{Z} / m$ and an arrow $i \mapsto i+1$ for each $i \in \mathbf{Z} / m$,

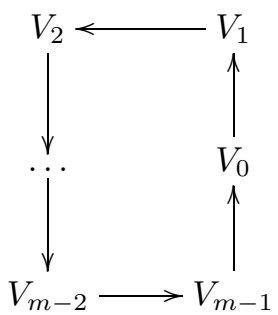

Then $\mathfrak{g}_{1}$ is the space of representations of $Q$ where we put $V_{i}$ at the vertex $i$.

More generally, if $G$ is a classical group, then the $G_{0^{-}}$-action on $\mathfrak{g}_{1}$ can be interpreted in terms of a cyclic quiver with some extra structure (see 9.5 for the case where $G$ is a symplectic group).

0.4. In this subsection we forget the $\mathbf{Z} / m$-grading. Instead of the action of $G_{\underline{0}}$ on $\mathfrak{g}_{i}$ and $\mathfrak{g}_{i}^{\text {nil }}$ we consider the adjoint action of $G$ on $\mathfrak{g}$ and on $\mathfrak{g}^{\text {nil }}$. Let $\mathcal{I}(\mathfrak{g})$ be the set of pairs $(\mathcal{O}, \mathcal{L})$ where $\mathcal{O}$ is a $G$-orbit on $\mathfrak{g}^{\text {nil }}$ and $\mathcal{L}$ is an irreducible $G$-equivariant local 
system on $\mathcal{O}$ (up to isomorphism). From the results on the generalized Springer theory in [L1] we have a canonical decomposition

$$
\mathcal{I}(\mathfrak{g})=\sqcup_{(L, C)} \mathcal{I}(\mathfrak{g})_{(L, C)},
$$

where $(L, C)$ runs over the $G$-conjugacy classes of data $L, C$ with $L$ a Levi subgroup of a parabolic subgroup of $G$ and $C$ an $L$-equivariant cuspidal perverse sheaf on the nilpotent cone of the Lie algebra of $L$. (Actually, the results of [L1 are stated for unipotent elements in $G$ instead of nilpotent elements in $\mathfrak{g}$.) We call (a) the block decomposition of $\mathcal{I}(\mathfrak{g})$.

Let $P\left(\mathfrak{g}^{\text {nil }}\right)$ be the subcategory of $\mathcal{D}\left(\mathfrak{g}^{\text {nil }}\right)$ consisting of complexes whose perverse cohomology sheaves are $G$-equivariant. Using (a) and [L3, (7.3.1)], we see that we have a direct sum decomposition

$$
P\left(\mathfrak{g}^{n i l}\right)=\oplus_{(L, C)} P\left(\mathfrak{g}^{n i l}\right)_{(L, C)},
$$

where $(L, C)$ is as in (a). We call (b) the block decomposition of $P\left(\mathfrak{g}^{\text {nil }}\right)$. In $[\mathrm{RR}]$ it is shown that the following variant of (b) holds: we have a direct sum decomposition

$$
\mathcal{D}_{G}\left(\mathfrak{g}^{n i l}\right)=\oplus_{(L, C)} \mathcal{D}_{G}\left(\mathfrak{g}^{n i l}\right)_{(L, C)},
$$

where $(L, C)$ is as in (a). We call (c) the block decomposition of $\mathcal{D}_{G}\left(\mathfrak{g}^{\text {nil }}\right)$.

In this paper we find a $\mathbf{Z} / m$-graded analogue of this (ungraded) block decomposition.

0.5. We fix $\zeta$, a primitive $m$-th root of 1 in $\mathbf{k}$ and we set $\vartheta=\tilde{\vartheta}(\zeta): G \rightarrow G$, $\theta=\tilde{\theta}(\zeta): \mathfrak{g} \rightarrow \mathfrak{g}$. Then for $i \in \mathbf{Z} / m$ we have $\mathfrak{g}_{i}=\left\{x \in \mathfrak{g} ; \theta(x)=\zeta^{i} x\right\}$.

Let $\eta \in \mathbf{Z}-\{0\}$. We consider systems $\left(M, \mathfrak{m}_{*}, \tilde{C}\right)$, where

$$
M=\{g \in G ; \operatorname{Ad}(\tau) \vartheta g=g\}
$$

for some semisimple element of finite order $\tau \in G_{\underline{0}}, \mathfrak{m}_{*}=\left\{\mathfrak{m}_{N}\right\}_{N \in \mathbf{Z}}$ is a $\mathbf{Z}$-grading of the Lie algebra $\mathfrak{m}$ of $M$ (see 0.11) such that $\mathfrak{m}_{N} \subset \mathfrak{g}_{N}$ for all $N, M_{0}$ is the closed connected subgroup of $M$ with Lie algebra $\mathfrak{m}_{0}$ and $\tilde{C}$ is an $M_{0}$-equivariant cuspidal perverse sheaf on $\mathfrak{m}_{\eta}$. We will review the notion of $M_{0}$-equivariant cuspidal perverse sheaf (already defined in [L4]) on $\mathfrak{m}_{\eta}$ in 1.2. Such a system $\left(M, \mathfrak{m}_{*}, \tilde{C}\right)$ is said to be admissible if a certain technical condition involving the group of components of the center of $M$ is satisfied (see 3.1).

Let $\underline{\mathfrak{T}}_{\eta}$ be the set of admissible systems up to $G_{\underline{0}}$-conjugacy. The following result is proved in 7.9 .

Theorem 0.6. There is a canonical direct sum decomposition of $\mathcal{D}_{G_{\underline{0}}}\left(\mathfrak{g}_{\underline{\eta}}^{\text {nil }}\right)$ into full subcategories

$$
\mathcal{D}_{G_{\underline{0}}}\left(\mathfrak{g}_{\underline{\eta}}^{n i l}\right)=\oplus_{\left(M, \mathfrak{m}_{*}, \tilde{C}\right) \in \mathfrak{T}_{\eta}} \mathcal{D}_{G_{\underline{0}}}\left(\mathfrak{g}_{\underline{\eta}}^{n i l}\right)_{\left(M, \mathfrak{m}_{*}, \tilde{C}\right)}
$$

indexed by $\underline{\mathfrak{T}}_{\eta}$.

In particular, any simple perverse sheaf in $\mathcal{D}_{G_{\underline{0}}}\left(\mathfrak{g}_{\underline{\eta}}^{\text {nil }}\right)$ belongs to a well-defined block $\mathcal{D}_{G_{\underline{0}}}\left(\mathfrak{g}_{\underline{\eta}}^{\text {nil }}\right)_{\left(M, \mathfrak{m}_{*}, \tilde{C}\right)}$. This gives a map

$$
\Psi: \mathcal{I}\left(\mathfrak{g}_{\underline{\eta}}\right) \rightarrow \underline{\mathfrak{T}}_{\eta} .
$$

In fact, we will first establish the map $\Psi$ in 3.5 and then prove the theorem in 7.9 , using a key calculation in Proposition 6.4. 
We also show in 3.9 and 7.8 that both the indexing set $\underline{\mathfrak{T}}_{\eta}$ and the blocks $\mathcal{D}_{G_{\underline{0}}}\left(\mathfrak{g}_{\underline{\eta}}^{\text {nil }}\right)_{\xi}$ (for $\xi \in \underline{\mathfrak{T}}_{\eta}$ ) only depend on the image $\underline{\eta} \in \mathbf{Z} / m$ and not on the integer $\bar{\eta}$.

Note that in the case where $m=1$, the theorem can be deduced from $0.4(\mathrm{a})$. On the other hand, for large $m$, a $\mathbf{Z} / m$-grading on $\mathfrak{g}$ is the same as a $\mathbf{Z}$-grading, so that in this case the theorem can be deduced from the results of [L4]. Thus, the result about block decomposition in this paper generalizes results in [L1] and [L4].

0.7. As an explicit example, let us consider the case where $G=S L_{n}(\mathbf{k}), \eta=1$. In the ungraded case, blocks are in bijection with pairs $(d, \chi)$ where $d$ is a divisor of $n$ and $\chi: \mu_{d} \rightarrow \overline{\mathbf{Q}}_{l}^{*}$ is a primitive character. (See [L1].) To $d$ we attach the subgroup $M=S\left(G L_{d}^{n / d}\right.$ ) (a Levi subgroup of a parabolic subgroup) and $\chi$ gives a cuspidal perverse sheaf $C_{\chi}$ with support equal to the nilpotent cone of the Lie algebra of $M$. Now in the $\mathbf{Z} / m$-graded case, we have $G=S L(V), V=\oplus_{i \in \mathbf{Z} / m} V_{i}$ as in 0.3 , and we identify $\mathfrak{g}_{1}$ with $\oplus_{i} \operatorname{Hom}\left(V_{i}, V_{i+1}\right)$. In this case, the set of blocks $\underline{\mathfrak{T}}_{1}$ has a similar explicit description. We have a natural bijection

$$
\underline{\mathfrak{T}}_{1} \leftrightarrow\{(d, f, \chi)\} / \sim .
$$

Here the right hand side is the set of equivalence classes of triples $(d, f, \chi)$ where $(d, \chi)$ is as in the ungraded case and $f:\{1,2, \ldots, n / d\} \rightarrow \mathbf{Z} / m$ is a map such that

$$
\sharp\{(b, y) \in \mathbf{Z} \times \mathbf{Z} ; 1 \leq b \leq n / d, 0 \leq y \leq d-1, f(b)+\underline{y}=i\}=\operatorname{dim} V_{i}
$$

for all $i \in \mathbf{Z} / m$. Two triples $(d, f, \chi)$ and $\left(d^{\prime}, f^{\prime}, \chi^{\prime}\right)$ are equivalent if and only if $d=d^{\prime}, \chi=\chi^{\prime}$ and $f^{\prime}$ is obtained from $f$ by composition with a permutation of $\{1,2, \ldots, n / d\}$.

0.8. In the ungraded case, the objects in the block $\mathcal{D}_{G}\left(\mathfrak{g}^{n i l}\right)_{(L, C)}$ are obtained from $C$ via parabolic induction (and decomposition) through any parabolic subgroup $P$ of $G$ containing $L$ as a Levi subgroup. In the $\mathbf{Z} / m$-graded case, a first attempt to generalize parabolic induction would be to start with a parabolic subgroup of $G$ compatible with the $\mathbf{Z} / m$-grading on $\mathfrak{g}$, as defined in the appendix of [L5]. However, such a parabolic induction does not produce all simple perverse sheaves in $\mathcal{D}_{G_{\underline{0}}}\left(\mathfrak{g}_{\eta}^{\text {nil }}\right)$. Instead, we introduce a certain induction procedure which we call spiral induction; see Section 4. We introduce the notion of a spiral $\mathfrak{p}_{*}$ which is a sequence of subspaces $\mathfrak{p}_{N} \subset \mathfrak{g}_{N}$, one for each $N \in \mathbf{Z}$, satisfying certain conditions; see Section 2. It turns out that spirals are the correct analogues of parabolic subalgebras in the $\mathbf{Z} / m$-graded case. Moreover, spiral induction includes the parabolic induction defined in the appendix of L5 as special cases. In fact there are two kinds of spiral inductions, one giving objects in $\mathcal{D}_{G_{\underline{0}}}\left(\mathfrak{g}_{\eta}^{\text {nil }}\right)$ and the other giving (assuming that $p>0$ ) Fourier-Deligne transforms of objects in $\mathcal{D}_{G_{0}}\left(\mathfrak{g}_{-\eta}^{\text {nil }}\right)$. The latter may be viewed as an analogue of character sheaves in the $\mathbf{Z} / m$-graded setting.

0.9. We now discuss the contents of the various sections. Many arguments in this paper rely on results from [L4] concerning $\mathbf{Z}$-graded Lie algebras; in Section 1 we review some results from [L4] that we need. In Section 2 we introduce the $\epsilon$-spirals attached to a $\mathbf{Z} / m$-graded Lie algebra and their splittings; the analogous concepts in the $\mathbf{Z}$-graded cases are the parabolic subalgebras compatible with the $\mathbf{Z}$-grading and their Levi subalgebras compatible with the $\mathbf{Z}$-grading. We also attach a canonical spiral to any element of $\mathfrak{g}_{\underline{\eta}}^{\text {nil }}$ which plays a crucial role in the arguments of this 
paper. In Section 3 we introduce the admissible systems, which eventually will be used to index the blocks in $\mathcal{D}_{G_{\underline{0}}}\left(\mathfrak{g}_{\underline{\eta}}^{\text {nil }}\right)$. In Section 4 we introduce the operation of spiral induction which is our main tool in the study of $\mathcal{D}_{G_{\underline{0}}}\left(\mathfrak{g}_{\underline{\eta}}^{\text {nil }}\right)$. In Sections 5 and 6 we calculate explicitly the Hom space between two spiral inductions, generalizing to the $\mathbf{Z} / m$-graded case a result from [L4]. This is used in Section 7 to prove Theorem 0.6. In Section 8 we introduce monomial and quasi-monomial complexes on $\mathfrak{g}_{\underline{\eta}}^{\text {nil }}$; we show that the monomial complexes (resp. quasi-monomial) complexes generate the appropriate Grothendieck group over $\mathbf{Q}(v)$ (resp. over $\mathbf{Z}\left[v, v^{-1}\right]$ ) where $v$ is an indeterminate; this again generalizes to the $\mathbf{Z} / m$-graded case a result from [L4]. This result is of the same type as that which says that the plus part of a quantized enveloping algebra defined in terms of perverse sheaves is generated over $\mathbf{Q}(v)$ by monomials in the $E_{i}$ and over $\mathbf{Z}\left[v, v^{-1}\right]$ by the products of divided powers of the $E_{i}$ (which could be called quasi-monomials). In Section 9 we discuss the examples where $G=S L(V)$ or $G=S p(V)$; in these cases we describe the spirals and in the case of $G=S L(V)$ we describe the blocks.

0.10. It is known that, in the ungraded case, each block of $\mathcal{D}_{G}\left(\mathfrak{g}^{\text {nil }}\right)$ can be related to the category of representations of a certain Weyl group; if $m$ is large, so that the $\mathbf{Z} / m$ grading of $\mathfrak{g}$ is a $\mathbf{Z}$-grading and $\mathfrak{g}_{\underline{\eta}}^{\text {nil }}=\mathfrak{g}_{\underline{\eta}}$, then each block of $\mathcal{D}_{G_{\underline{0}}}\left(\mathfrak{g}_{\underline{\eta}}^{\text {nil }}\right)$ is related to the category of representations of a certain graded affine Hecke algebra with possibly unequal parameters. In fact, without assumptions on $m$, each block of $\mathcal{D}_{G_{\underline{0}}}\left(\mathfrak{g}_{\eta}^{\text {nil }}\right)$ is related to a certain graded double affine Hecke algebra (corresponding to an affine Weyl group attached to the block) with possibly unequal parameters; this will be considered in a sequel to this paper. We also plan to describe explicitly the blocks in the case where $G$ is a classical group and relate them to cyclic quivers with extra structure. The case of the symplectic group is partially carried out in 9.5-9.7.

0.11. Notation. All algebraic varieties are assumed to be over $\mathbf{k}$; all algebraic groups are assumed to be affine. Let $l$ be a prime number invertible in $\mathbf{k}$. For any algebraic variety $X$ we denote by $\mathcal{D}(X)$ the bounded derived category of $\overline{\mathbf{Q}}_{l^{-}}$ complexes on $X$. Let $D: \mathcal{D}(X) \rightarrow \mathcal{D}(X)$ be Verdier duality.

For $K \in \mathcal{D}(X)$ we denote by $\mathcal{H}^{n} K$ the $n$-th cohomology sheaf of $K$ and by $\mathcal{H}_{x}^{n} K$ the stalk of $\mathcal{H}^{n} K$ at $x \in X$.

If $X^{\prime}$ is a locally closed smooth irreducible subvariety of $X$ with closure $\bar{X}^{\prime}$ and $\mathcal{L}$ is an irreducible local system on $X^{\prime}$ we denote by $\mathcal{L}^{\sharp} \in \mathcal{D}(X)$ the intersection cohomolgy complex of $\bar{X}^{\prime}$ with coefficients in $\mathcal{L}$, extended by 0 on $X-\bar{X}^{\prime}$.

If $X$ has a given action of an algebraic group $H$ we denote by $\mathcal{D}_{H}(X)$ the corresponding equivariant derived category.

If $H$ is an algebraic group we denote by $H^{0}$ the identity component of $H$, by $\mathcal{Z}_{H}$ the center of $H$. We set $\pi_{0}(H)=H / H^{0}$. Now assume that $H$ is connected. We denote by $\mathfrak{L} H$ the Lie algebra of $H$ and by $U_{H}$ the unipotent radical of $H$. Let $\mathfrak{h}=\mathfrak{L} H$. If $\mathfrak{h}^{\prime}$ is a Lie subalgebra of $\mathfrak{h}$ we write $e^{\mathfrak{h}^{\prime}} \subset H$ for the closed connected subgroup of $H$ such that $\mathfrak{L}\left(e^{\mathfrak{h}^{\prime}}\right)=\mathfrak{h}^{\prime}$, assuming that such a subgroup exists.

We shall often denote a collection $\left\{V_{N} ; N \in \mathbf{Z}\right\}$ of vector spaces indexed by $N \in \mathbf{Z}$ by the symbol $V_{*}$.

If $V$ is a $\mathbf{k}$-vector space, a $\mathbf{Z}$-grading on $V$ is a collection of subspaces $V_{*}=$ $\left\{V_{k} ; k \in \mathbf{Z}\right\}$ such that $V=\oplus_{k \in \mathbf{Z}} V_{k} ;$ a $\mathbf{Z} / m$-grading on $V$ is a collection of subspaces 
$\left\{V_{i} ; i \in \mathbf{Z} / m\right\}$ such that $V=\oplus_{i \in \mathbf{Z} / m} V_{i}$; a Q-grading on $V$ is a collection of subspaces $\left\{{ }_{\kappa} V ; \kappa \in \mathbf{Q}\right\}$ such that $V=\oplus_{\kappa \in \mathbf{Q}}\left({ }_{\kappa} V\right)$.

A $\mathbf{Z}$-grading for the Lie algebra $\mathfrak{h}$ is a $\mathbf{Z}$-grading $\mathfrak{h}_{*}=\left\{\mathfrak{h}_{k} ; k \in \mathbf{Z}\right\}$ of $\mathfrak{h}$ as a vector space satisfying $\left[\mathfrak{h}_{k}, \mathfrak{h}_{k^{\prime}}\right] \subset \mathfrak{h}_{k+k^{\prime}}$ for all $k, k^{\prime} \in \mathbf{Z}$; a $\mathbf{Z} / m$-grading for $\mathfrak{h}$ is a $\mathbf{Z} / m$-grading $\left\{\mathfrak{h}_{i} ; i \in \mathbf{Z} / m\right\}$ of $\mathfrak{h}$ as a vector space satisfying $\left[\mathfrak{h}_{i}, \mathfrak{h}_{i^{\prime}}\right] \subset \mathfrak{h}_{i+i^{\prime}}$ for all $i, i^{\prime} \in \mathbf{Z} / m$; a $\mathbf{Q}$-grading for $\mathfrak{h}$ is a $\mathbf{Q}$-grading $\left\{{ }_{\kappa} \mathfrak{h} ; \kappa \in \mathbf{Q}\right\}$ of $\mathfrak{h}$ as a vector space satisfying $\left[\kappa \mathfrak{h}, \kappa^{\prime} \mathfrak{h}\right] \subset \kappa+\kappa^{\prime} \mathfrak{h}$ for all $\kappa, \kappa^{\prime} \in \mathbf{Q}$.

Let $Y_{H}$ be the set of homomorphisms of algebraic groups $\mathbf{k}^{*} \rightarrow H$. For $\lambda \in Y_{H}$ and $c \in \mathbf{Z}$, we define $c \lambda \in Y_{H}$ by $(c \lambda)(t)=\lambda\left(t^{c}\right)$ for $t \in \mathbf{k}^{*}$. We define an equivalence relation on $Y_{H} \times \mathbf{Z}_{>0}$ by $(\lambda, r) \sim\left(\lambda^{\prime}, r^{\prime}\right)$ whenever there exist $c, c^{\prime}$ in $\mathbf{Z}_{>0}$ such that $c \lambda=c^{\prime} \lambda^{\prime}, c r=c^{\prime} r^{\prime}$; the set of equivalence classes for this relation is denoted by $Y_{H, \mathbf{Q}}$. Let $\lambda / r=(1 / r) \lambda$ be the equivalence class of $(\lambda, r)$. Now $\lambda \mapsto \lambda / 1$ identifies $Y_{H}$ with a subset of $Y_{H, \mathbf{Q}}$. For $\kappa \in \mathbf{Q}, \mu \in Y_{H, \mathbf{Q}}$ we define $\kappa \mu \in Y_{H, \mathbf{Q}}$ by $\kappa \mu=(k \lambda) /\left(k^{\prime} r\right)$, where $k \in \mathbf{Z}, k^{\prime} \in \mathbf{Z}_{>0}, r \in \mathbf{Z}_{>0}, \lambda \in Y_{H}$ are such that $\kappa=k / k^{\prime}$, $\mu=\lambda / r$; this is independent of the choices. In particular, we have $r \mu \in Y_{H}$ for some $r \in \mathbf{Z}_{>0}$.

Let $\lambda \in Y_{H}$. For $k \in \mathbf{Z}$ we set

$$
{ }_{k}^{\lambda} \mathfrak{h}=\left\{x \in \mathfrak{h} ; \operatorname{Ad}(\lambda(t)) x=t^{k} x \quad \forall t \in \mathbf{k}^{*}\right\} .
$$

Note that $\left\{\begin{array}{l}\lambda \\ k\end{array} \mathfrak{h}, k \in \mathbf{Z}\right\}$ is a $\mathbf{Z}$-grading of $\mathfrak{h}$.

Now let $\mu \in Y_{H, \mathbf{Q}}$. For $\kappa \in \mathbf{Q}$ we set ${ }_{\kappa}^{\mu} \mathfrak{h}={ }_{r \kappa}^{r \mu} \mathfrak{h}$ where $r \in \mathbf{Z}_{>0}$ is chosen so that $r \mu \in Y_{H}, r \kappa \in \mathbf{Z}$. This is well defined (independent of the choice of $r$ ). Note that $\left\{{ }_{\kappa}^{\mu} \mathfrak{h}, \kappa \in \mathbf{Q}\right\}$ is a $\mathbf{Q}$-grading of $\mathfrak{h}$.

0.12. Let $H$ be a connected algebraic group acting on an algebraic variety $X$ and let $A, B$ be two $H$-equivariant semisimple complexes on $X$; let $j \in \mathbf{Z}$. We define a finite dimensional $\overline{\mathbf{Q}}_{l}$-vector space $\mathbf{D}_{j}(X, H ; A, B)$ as in [L4, 1.7]. For the purpose of this paper, we can take the following formula as the definition of $\mathbf{D}_{j}(X, H ; A, B)$ :

(a) $\mathbf{D}_{j}(X, H ; A, B)=\operatorname{Hom}_{\mathcal{D}_{H}(X)}(A, D(B)[-j])^{*}$.

Let $d_{j}(X ; A, B)=\operatorname{dim} \mathbf{D}_{j}(X, H ; A, B),\{A, B\}=\sum_{j \in \mathbf{Z}} d_{j}(X ; A, B) v^{-j} \in \mathbf{N}((v))$ where $v$ is an indeterminate.

If $A, B$ are $H$-equivariant simple perverse sheaves on $X$, then

$$
\begin{aligned}
& \{A, B\} \in 1+v \mathbf{N}[[v]] \text { if } B \cong D(A), \\
& \{A, B\} \in v \mathbf{N}[[v]] \text { if } B \not D(A) .
\end{aligned}
$$

(See [L4, 1.8(d)].)

For an algebraic variety $X$ we denote by $\rho_{X}$ the map $X \rightarrow$ (point).

Let $v$ be an indeterminate and let $\mathcal{A}=\mathbf{Z}\left[v, v^{-1}\right]$. Let $^{-}: \mathbf{Q}(v) \rightarrow \mathbf{Q}(v)$ be the field involution such that $\bar{v}=v^{-1}$. This restricts to a ring involution ${ }^{-}: \mathcal{A} \rightarrow \mathcal{A}$.

For any $\eta \in \mathbf{Z}-\{0\}$ we define $\dot{\eta}=\eta /|\eta| \in\{1,-1\}$ where $|\eta|$ is the absolute value of $\eta$.

\section{Recollections on Z-GRAded Lie algebras}

In this section we recall notation and results from [L4 that will be used in this paper. 
1.1. In this section we fix a connected reductive group $H$; let $\mathfrak{h}=\mathfrak{L} H$.

Let $J^{H}$ be the variety consisting of all triples $(e, h, f) \in \mathfrak{h}^{3}$ such that $[h, e]=$ $2 e,[h, f]=-2 f,[e, f]=h$ (then $e, f$ are necessarily in $\mathfrak{h}^{n i l}$ ). If $\phi=(e, h, f) \in J^{H}$, there is a unique homomorphism of algebraic groups $\tilde{\phi}: S L_{2}(\mathbf{k}) \rightarrow H$ such that the differential of $\tilde{\phi}$ carries $\left(\begin{array}{cc}0 & 1 \\ 0 & 0\end{array}\right),\left(\begin{array}{cc}1 & 0 \\ 0 & -1\end{array}\right),\left(\begin{array}{cc}0 & 0 \\ 1 & 0\end{array}\right)$ to $e, h, f$ respectively; we then define $\iota_{\phi} \in Y_{H}$ by $\iota_{\phi}(t)=\tilde{\phi}\left(\begin{array}{cc}t & 0 \\ 0 & t^{-1}\end{array}\right)$.

1.2. In the remainder of this section we assume that a $\mathbf{Z}$-grading $\mathfrak{h}_{*}$ for $\mathfrak{h}$ is given. Then there exists $\lambda \in Y_{H}$ and $r \in \mathbf{Z}_{>0}$ with $\mathfrak{h}_{k}={ }_{r k}^{\lambda} \mathfrak{h}$ for all $k \in \mathbf{Z}$. (It follows that ${ }_{\kappa}^{\lambda} \mathfrak{h}=0$ for all $\kappa \in \mathbf{Q}-r \mathbf{Z}$.)

(In this paper we will often refer to results in [L4, even though, strictly speaking, in [L4] a stronger assumption on the $\mathbf{Z}$-grading of $\mathfrak{h}$ is made, namely that $r$ above can be taken to be 1 . Note that the results of [L4] hold with the same proof when the stronger assumption is replaced by the present assumption.)

We have $\mathfrak{h}_{k} \subset \mathfrak{h}^{\text {nil }}$ for any $k \in \mathbf{Z}-\{0\}$. Note that $\mathfrak{h}_{0}$ is a Lie subalgebra of $\mathfrak{h}$ and that $H_{0}:=e^{\mathfrak{h}_{0}} \subset H$ is well defined and it acts by the Ad-action on each $\mathfrak{h}_{k}$. If $k \neq 0$, this action has only finitely many orbits (see [L4, 3.5]); we denote by $\mathfrak{h}_{k}$ the unique open $H_{0}$-orbit in $\mathfrak{h}_{k}$.

Let $\eta \in \mathbf{Z}-\{0\}$.

(a) We say that the $\mathbf{Z}$-grading $\mathfrak{h}_{*}$ of $\mathfrak{h}$ is $\eta$-rigid if there exists $\iota \in Y_{H}$ such that (i), (ii) below hold.

(i) ${ }_{k}^{\iota} \mathfrak{h}=\mathfrak{h}_{\eta k / 2}$ for any $k \in \mathbf{Z}$ such that $\eta k / 2 \in \mathbf{Z}$ and ${ }_{k}^{\iota} \mathfrak{h}=0$ for any $k \in \mathbf{Z}$ such that $\eta k / 2 \notin \mathbf{Z}$;

(ii) $\iota=\iota_{\phi}$ for some $\phi=(e, h, f) \in J^{H}$ such that $e \in \mathfrak{\circ}_{\eta}, h \in \mathfrak{h}_{0}, f \in \mathfrak{h}_{-\eta}$. It follows that $2 k^{\prime} \in \eta \mathbf{Z}$ whenever $\mathfrak{h}_{k^{\prime}} \neq 0$. Note that $\iota$ is unique if it exists, since, by (ii), $\iota\left(\mathbf{k}^{*}\right)$ is contained in the derived group of $H$.

We show:

(b) In the setup of (a), let $\phi^{\prime}=\left(e^{\prime}, h^{\prime}, f^{\prime}\right) \in J^{H}$ be such that $e^{\prime} \in \grave{\mathfrak{h}}_{\eta}, h^{\prime} \in \mathfrak{h}_{0}$, $f^{\prime} \in \mathfrak{h}_{-\eta}$. Let $\iota^{\prime}=\iota_{\phi^{\prime}}$. Then $\iota^{\prime}=\iota$.

Let $\phi$ be as in (ii). Using [L4, 3.3], we can find $g_{0} \in H_{0}$ such that $\operatorname{Ad}\left(g_{0}\right)$ carries $\phi$ to $\phi^{\prime}$. It follows that $\operatorname{Ad}\left(g_{0}\right) \iota(t)=\iota^{\prime}(t)$ for any $t \in \mathbf{k}^{*}$. For $k \in \mathbf{Z}$ such that $\eta k / 2 \in \mathbf{Z}$ we have

$$
{ }_{k}^{\iota^{\prime}} \mathfrak{h}=\operatorname{Ad}\left(g_{0}\right)\left({ }_{k}^{\iota} \mathfrak{h}\right)=\operatorname{Ad}\left(g_{0}\right) \mathfrak{h}_{k}=\mathfrak{h}_{k} ;
$$

for $k \in \mathbf{Z}$ such that $\eta k / 2 \notin \mathbf{Z}$ we have

$$
\begin{aligned}
& { }_{k}^{\iota^{\prime}} \mathfrak{h}=\operatorname{Ad}\left(g_{0}\right)\left({ }_{k}^{\iota} \mathfrak{h}\right)=0, \\
& {\stackrel{\iota}{\prime}{ }^{\prime}}^{\prime} \mathfrak{h}=\operatorname{Ad}\left(g_{0}\right)\left({ }_{2 k \eta}^{\iota} \mathfrak{h}\right)=\operatorname{Ad}\left(g_{0}\right) \mathfrak{h}_{k}=\mathfrak{h}_{k} .
\end{aligned}
$$

Thus $\iota^{\prime}$ satisfies the defining properties of $\iota$ in (a). By uniqueness we have $\iota^{\prime}=\iota$ as required.

Let $\mathcal{I}\left(\mathfrak{h}_{\eta}\right)$ be the set of all pairs $(\mathcal{O}, \mathcal{L})$ where $\mathcal{O}$ is an $H_{0}$-orbit in $\mathfrak{h}_{\eta}$ and $\mathcal{L}$ is an $H_{0}$-equivariant irreducible local system on $\mathfrak{h}_{\eta}$ (up to isomorphism).

Let $\mathcal{Q}\left(\mathfrak{h}_{\eta}\right)$ be the category of $\overline{\mathbf{Q}}_{l}$-complexes on $\mathfrak{h}_{\eta}$ which are direct sums of shifts of simple $H_{0}$-equivariant perverse sheaves on $\mathfrak{h}_{\eta}$. There are up to isomorphism only finitely many such simple perverse sheaves; they form a set in bijection with $\mathcal{I}\left(\mathfrak{h}_{\eta}\right)$.

An $H_{0}$-equivariant perverse sheaf $A$ on $\mathfrak{h}_{\eta}$ is said to be cuspidal if there exists a nilpotent $H$-orbit $\mathcal{C}$ in $\mathfrak{h}$ and an irreducible $H$-equivariant cuspidal local system $\mathcal{F}$ 
on $\mathcal{C}$ such that $\stackrel{\circ}{\mathfrak{h}}_{\eta} \subset \mathcal{C}$ and $\left.A\right|_{\mathfrak{h}_{\eta}}=\left.\mathcal{F}\right|_{\mathfrak{h}_{\eta}}\left[\operatorname{dim} \mathfrak{h}_{\eta}\right]$. If such $(\mathcal{C}, \mathcal{F})$ exists, it is unique; see [L4, 4.2(c)]. Note that if $A$ is cuspidal, then it is necessarily a simple perverse sheaf.

(c) If there exists a cuspidal $H_{0}$-equivariant perverse sheaf $A$ on $\mathfrak{h}_{\eta}$, the grading $\mathfrak{h}_{*}$ of $\mathfrak{h}$ is necessarily $\eta$-rigid; moreover, we have $\left.A\right|_{\mathfrak{h}_{\eta}-\mathfrak{h}_{\eta}}=0$.

(See [L4, 4.4(a), 4.4(b)].)

In the setup of (c), the element $\iota \in Y_{H}$ provided by (a) is known to satisfy

(d) ${ }_{k}^{\iota} \mathfrak{h}=0$ unless $k \in 2 \mathbf{Z}$;

we deduce that:

(e) If $k^{\prime} \in \mathbf{Z}$ and $\mathfrak{h}_{k^{\prime}} \neq 0$, then $k^{\prime} / \eta \in \mathbf{Z}$.

1.3. Parabolic induction. In the setup of 1.2 assume that $P$ is a parabolic subgroup of $H$ with $\mathfrak{p}:=\mathfrak{L} P$ satisfying $\mathfrak{p}=\oplus_{k \in \mathbf{Z}} \mathfrak{p}_{k}$ where $\mathfrak{p}_{k}=\mathfrak{p} \cap \mathfrak{h}_{k}$. We set $U=U_{P}, L=P / U, \mathfrak{u}=\mathfrak{L} U, \mathfrak{l}=\mathfrak{L} L=\mathfrak{p} / \mathfrak{u}$. We have $\mathfrak{u}=\oplus_{k \in \mathbf{Z}} \mathfrak{u}_{k}$ where $\mathfrak{u}_{k}=\mathfrak{u} \cap \mathfrak{h}_{k}$. Setting $\mathfrak{l}_{k}=\mathfrak{p}_{k} / \mathfrak{u}_{k}$, we have $\mathfrak{l}=\oplus_{k \in \mathbf{Z}} \mathfrak{l}_{k}$; this gives a $\mathbf{Z}$-grading of the Lie algebra $\mathfrak{l}$.

Now $\mathfrak{p}_{0}$ is a parabolic subalgebra of the reductive Lie algebra $\mathfrak{h}_{0}$; we have $\mathfrak{p}_{0}=$ $\mathfrak{L} P_{0}$ where $P_{0}$ is a parabolic subgroup of the connected reductive group $H_{0}$. Let $L_{0}$ be the image of $P_{0}$ under the obvious homomorphism $P \rightarrow L$. Then $L_{0}=e^{\mathfrak{l}_{0}} \subset L$. Now $P_{0}$ acts by the Ad-action on each $\mathfrak{p}_{k}$. Let $\pi: \mathfrak{p}_{\eta} \rightarrow \mathfrak{l}_{\eta}$ be the obvious projection. We have a diagram

$$
\mathfrak{l}_{\eta} \stackrel{a}{\leftarrow} H_{0} \times \mathfrak{p}_{\eta} \stackrel{b}{\rightarrow} E \stackrel{c}{\rightarrow} \mathfrak{h}_{\eta},
$$

where

$$
\begin{aligned}
E & =\left\{\left(h P_{0}, z\right) \in H_{0} / P_{0} \times \mathfrak{h}_{\eta} ; \operatorname{Ad}\left(h^{-1}\right) z \in \mathfrak{p}_{\eta}\right\}, \\
a(h, z) & =\pi\left(\operatorname{Ad}\left(h^{-1}\right) z\right), b(h, z)=\left(h P_{0}, z\right), c\left(g P_{0}, z\right)=z .
\end{aligned}
$$

Now $a$ is smooth with connected fibers, $b$ is a principal $P_{0}$-bundle and $c$ is proper. If $A \in \mathcal{Q}\left(\mathfrak{l}_{\eta}\right)$, then $a^{*} A$ is a $P_{0}$-equivariant semisimple complex on $H_{0} \times \mathfrak{p}_{\eta}$ hence there is a well-defined semisimple complex $A_{1}$ on $E$ such that $b^{*} A_{1}=a^{*} A$. Since $c$ is proper, the complex

$$
\operatorname{ind}_{\mathfrak{p}_{\eta}}^{\mathfrak{h}_{\eta}}(A):=c_{!} A_{1}
$$

belongs to $\mathcal{Q}\left(\mathfrak{h}_{\eta}\right)$. For $B \in \mathcal{D}\left(\mathfrak{h}_{\eta}\right)$ we can form

$$
\operatorname{res}_{\mathfrak{p}_{\eta}}^{\mathfrak{h}_{\eta}}(B):=\pi_{!}\left(\left.B\right|_{\mathfrak{p}_{\eta}}\right) \in \mathcal{D}\left(\mathfrak{l}_{\eta}\right) \text {. }
$$

Thus we have functors $\operatorname{res}_{\mathfrak{p}_{\eta}}^{\mathfrak{h}_{\eta}}: \mathcal{D}\left(\mathfrak{h}_{\eta}\right) \rightarrow \mathcal{D}\left(\mathfrak{l}_{\eta}\right), \operatorname{ind}_{\mathfrak{p}_{\eta}}^{\mathfrak{h}_{\eta}}: \mathcal{Q}\left(\mathfrak{l}_{\eta}\right) \rightarrow \mathcal{Q}\left(\mathfrak{h}_{\eta}\right)$.

When $\tilde{\mathfrak{l}}$ is a Levi subalgebra of $\mathfrak{p}$ such that $\tilde{\mathfrak{l}}=\oplus_{k \in \mathbf{Z}} \tilde{\mathfrak{l}}_{k}$ with $\tilde{\mathfrak{l}}_{k}=\tilde{\mathfrak{l}} \cap \mathfrak{h}_{k}$, we will sometime consider $\operatorname{ind}_{\mathfrak{p}_{\eta}}^{\mathfrak{h}_{\eta}}(A)$ with $A \in \mathcal{Q}\left(\tilde{\mathfrak{l}}_{\eta}\right)$ by identifying $\tilde{\mathfrak{l}}_{\eta}=\mathfrak{l}_{\eta}$ in an obvious way and $A$ with an object in $\mathcal{Q}\left(\mathfrak{l}_{\eta}\right)$.

1.4. In the setup of 1.3 let $S_{P}^{\prime}$ be the set of Levi subgroups of $P$ and let $S_{P}$ be the set of all $M \in S_{P}^{\prime}$ such that, setting $\mathfrak{L} M=\mathfrak{m}, \mathfrak{m}_{k}=\mathfrak{m} \cap \mathfrak{h}_{k}$, we have $\mathfrak{m}=\oplus_{k \in \mathbf{Z}} \mathfrak{m}_{k}$, or equivalently such that $\operatorname{Ad}(\lambda(t)) \mathfrak{m}=\mathfrak{m}$ for all $t \in \mathbf{k}^{*}$. We have $S_{P} \neq \emptyset$; indeed, we can find $M \in S_{P}^{\prime}$ such that $\lambda\left(k^{*}\right) \subset M$; then $M \in S_{P}$. Since $U$ acts simply transitively by conjugation on $S_{P}^{\prime}$, it follows that:

(a) The unipotent group $\left\{u \in U ; u \lambda(t)=\lambda(t) u \quad \forall t \in \mathbf{k}^{*}\right\}$ acts simply transitively by conjugation on $S_{P}$. 
1.5. Blocks for $\mathcal{Q}\left(\mathfrak{h}_{\eta}\right)$. Let $\mathfrak{M}_{\eta}(H)$ be the set of all systems

$$
\left(M, M_{0}, \mathfrak{m}, \mathfrak{m}_{*}, \tilde{C}\right),
$$

where $M$ is a Levi subgroup of some parabolic subgroup of $H, \mathfrak{m}=\mathfrak{L} M, \mathfrak{m}_{*}$ is a Z-grading of $\mathfrak{m}$ such that $\mathfrak{m}_{k}=\mathfrak{m} \cap \mathfrak{h}_{k}$ for all $k, M_{0}=e^{\mathfrak{m}_{0}} \subset M$ and $\tilde{C}$ is a cuspidal $M_{0}$-equivariant perverse sheaf on $\mathfrak{m}_{\eta}$ (up to isomorphism). Note that $H_{0}$ acts by conjugation on $\mathfrak{M}_{\eta}(H)$. Let $\underline{\mathfrak{M}}_{\eta}(H)$ be the set of orbits for this action.

In the setup of 1.2 assume that $A$ is a simple $H_{0}$-equivariant perverse sheaf on $\mathfrak{h}_{\eta}$. By [L4, 7.5]:

(a) There exists $P, L, L_{0}, \mathfrak{p}, \mathfrak{l}$ as in 1.3 and a cuspidal $L_{0}$-equivariant perverse sheaf $C$ on $\mathfrak{l}_{\eta}$ such that some shift of $A$ is a direct summand of $\operatorname{ind}_{\mathfrak{p}_{\eta}}^{\mathfrak{h}_{\eta}}(C)$.

Assume now that $P^{\prime}, L^{\prime}, L_{0}^{\prime}, \mathfrak{p}^{\prime}, \mathfrak{l}^{\prime}$ is another quintuple like $P, L, L_{0}, \mathfrak{p}, \mathfrak{l}$ and that $C^{\prime}$ is a cuspidal $L_{0}^{\prime}$-equivariant perverse sheaf on $\mathfrak{l}_{\eta}^{\prime}$ such that some shift of $A$ is a direct summand of $\operatorname{ind}_{\mathfrak{p}_{\eta}^{\prime}}^{\mathfrak{h}_{\eta}}\left(C^{\prime}\right)$.

Let $M \in S_{P}, M^{\prime} \in S_{P^{\prime}}$, let $\mathfrak{L} M=\mathfrak{m}=\oplus_{k} \mathfrak{m}_{k}$ be as in 1.4 and let $\mathfrak{L} M^{\prime}=\mathfrak{m}^{\prime}=$ $\oplus_{k} \mathfrak{m}_{k}^{\prime}$ where $\mathfrak{m}_{k}^{\prime}=\mathfrak{m}^{\prime} \cap \mathfrak{h}_{k}$. Let $M_{0}=e^{\mathfrak{m}_{0}} \subset M, M_{0}^{\prime}=e^{\mathfrak{m}_{0}^{\prime}} \subset M^{\prime}$. We can identify $M, M_{0}, \mathfrak{m}, \mathfrak{m}_{k}$ with $L, L_{0}, \mathfrak{l}, \mathfrak{l}_{k}$ via $P \rightarrow L$ and we can identify $M^{\prime}, M_{0}^{\prime}, \mathfrak{m}^{\prime}, \mathfrak{m}_{k}^{\prime}$ with $L^{\prime}, L_{0}^{\prime}, \mathfrak{l}^{\prime}, \mathfrak{l}_{k}^{\prime}$ via $P^{\prime} \rightarrow L^{\prime}$. Then $C$ (resp. $C^{\prime}$ ) becomes a cuspidal $M_{0}$-equivariant (resp. $M_{0}^{\prime}$-equivariant) perverse sheaf $\tilde{C}$ (resp. $\left.\tilde{C}^{\prime}\right)$ on $\mathfrak{m}_{\eta}$ (resp. $\left.\mathfrak{m}_{\eta}^{\prime}\right)$.

Using the last sentence of [L4, 15.3], we see that there exists $h \in H_{0}$ such that $\operatorname{Ad}(h)$ carries $M, M_{0}, \mathfrak{m}, \mathfrak{m}_{k}$ to $M^{\prime}, M_{0}^{\prime}, \mathfrak{m}^{\prime}, \mathfrak{m}_{k}^{\prime}$ and $\tilde{C}$ to $\tilde{C}^{\prime}$. Thus, we have:

(b) $A \mapsto\left(M, M_{0}, \mathfrak{m}, \mathfrak{m}_{k}, \tilde{C}\right)$ is a well-defined map from the set of (isomorphism classes) of simple $H_{0}$-equivariant perverse sheaves on $\mathfrak{h}_{\eta}$ to the set $\underline{\mathfrak{M}}_{\eta}(H)$.

1.6. Let $\left(M, M_{0}, \mathfrak{m}, \mathfrak{m}_{k}, \tilde{C}\right) \in \mathfrak{M}_{\eta}(H)$. We show:

(a) There exists a parabolic subgroup $P$ of $H$ such that $M$ is a Levi subgroup of $P$ and such that, setting $\mathfrak{p}=\mathfrak{L} P, \mathfrak{p}_{k}=\mathfrak{p} \cap \mathfrak{h}_{k}$, we have $\mathfrak{p}=\oplus_{k \in \mathbf{Z}} \mathfrak{p}_{k}$.

Let $\mathcal{Z}=\mathcal{Z}_{M}^{0}$. Then $\mathfrak{z}=\mathfrak{L} \mathcal{Z}$ is the center of $\mathfrak{m}$. Since $\mathfrak{m}_{0}$ is a Levi subalgebra of a parabolic subalgebra of $\mathfrak{m}$, we have $\mathfrak{z} \subset \mathfrak{m}_{0}$ hence $\mathcal{Z} \subset M_{0}$. We can find $\lambda_{1} \in Y_{\mathcal{Z}}$ such that the centralizer of $\lambda_{1}\left(\mathbf{k}^{*}\right)$ in $H$ is equal to the centralizer of $\mathcal{Z}$ in $H$ which equals $M$. Let $\lambda \in Y_{H}, r$ be as in 1.2. Then $\lambda\left(\mathbf{k}^{*}\right) \subset \mathcal{Z}_{H_{0}}$. Now $\lambda_{1}\left(\mathbf{k}^{*}\right) \subset \mathcal{Z}$ hence $\lambda_{1}\left(\mathbf{k}^{*}\right) \subset H_{0}$. It follows that $\lambda_{1}(t) \lambda\left(t^{\prime}\right)=\lambda\left(t^{\prime}\right) \lambda_{1}(t)$ for any $t, t^{\prime}$ in $\mathbf{k}^{*}$. Thus we

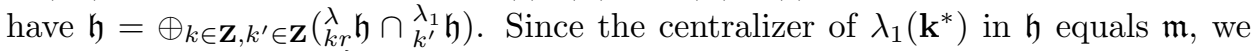
have $\left.\mathfrak{m}=\oplus_{k \in \mathbf{Z}}\left(\begin{array}{c}\lambda \\ k r\end{array}\right) \cap{ }_{0}^{\lambda_{1}} \mathfrak{h}\right)$. We set

$$
\left.\mathfrak{p}=\oplus_{k \in \mathbf{Z}, k^{\prime} \in \mathbf{Z}_{\geq 0}}\left(\begin{array}{l}
\lambda \\
k r
\end{array}\right) \cap \begin{array}{l}
\lambda_{1} \\
k^{\prime}
\end{array}\right) .
$$

Clearly, $\mathfrak{p}$ is a parabolic subalgebra of $\mathfrak{h}$ with Levi subalgebra $\mathfrak{m}$ and such that, setting $\mathfrak{p}_{k}=\mathfrak{p} \cap \mathfrak{h}_{k}$, we have $\mathfrak{p}=\oplus_{k \in \mathbf{Z}} \mathfrak{p}_{k}$. This proves (a).

1.7. To any $\left(M, M_{0}, \mathfrak{m}, \mathfrak{m}_{*}, \tilde{C}\right) \in \mathfrak{M}_{\eta}(H)$ we associate a simple perverse sheaf $A$ in $\mathcal{Q}\left(\mathfrak{h}_{\eta}\right)$ as follows. Let $\mathcal{O}$ be the $H_{0}$-orbit in $\mathfrak{h}_{\eta}$ which contains $\stackrel{\circ}{\mathfrak{m}}_{\eta}$. Let $\mathcal{L}^{\prime}$ be the

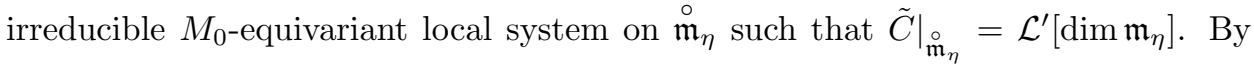
[L4, 11.2], there is a well-defined irreducible $H_{0}$-equivariant local system $\mathcal{L}$ on $\mathcal{O}$ such that $\left.\mathcal{L}\right|_{\mathfrak{m}_{\eta}}=\mathcal{L}^{\prime}$. By definition, $A$ is the simple perverse sheaf on $\mathfrak{h}_{\eta}$ such that $\operatorname{supp} A$ is contained in the closure of $\mathcal{O}$ and $\left.A\right|_{\mathcal{O}}=\mathcal{L}[\operatorname{dim} \mathcal{O}]$. 
1.8. Assume that the $\mathbf{Z}$-grading $\mathfrak{h}_{*}$ of $\mathfrak{h}$ is $\eta$-rigid. A perverse sheaf $A$ in $\mathcal{Q}\left(\mathfrak{h}_{\eta}\right)$ is said to be $\eta$-semicuspidal if $\operatorname{supp} A=\mathfrak{h}_{\eta}$ and $A$ is attached to some

$$
\left(M, M_{0}, \mathfrak{m}, \mathfrak{m}_{*}, \tilde{C}\right) \in \mathfrak{M}_{\eta}(H),
$$

as in 1.7 (in particular, $A$ is a simple perverse sheaf). In this case we have $\stackrel{\circ}{\mathfrak{m}}_{\eta} \subset \stackrel{\circ}{\mathfrak{h}}_{\eta}$; moreover,

(a) $H_{0}$ acts transitively on the set of systems $\left(M, M_{0}, \mathfrak{p}, \mathfrak{p}_{*}, \mathfrak{m}, \mathfrak{m}_{*}, \tilde{C}\right)$ such that $\left(M, M_{0}, \mathfrak{m}, \mathfrak{m}_{*}, \tilde{C}\right) \in \mathfrak{M}_{\eta}(H), A$ is attached to $\left(M, M_{0}, \mathfrak{m}, \mathfrak{m}_{*}, \tilde{C}\right)$ as in $1.7, \mathfrak{p}$ is a parabolic subalgebra of $\mathfrak{h}$ with Levi subalgebra $\mathfrak{m}$ and $\mathfrak{p}=\oplus_{k \in \mathbf{Z}} \mathfrak{p}_{k}$ where $\mathfrak{p}_{k}=\mathfrak{p} \cap \mathfrak{h}_{k}$. (See [L4, 11.9].)

If $\left(M, M_{0}, \mathfrak{p}, \mathfrak{p}_{*}, \mathfrak{m}, \mathfrak{m}_{*}, \tilde{C}\right)$ is as in (a), then

$$
\operatorname{ind}_{\mathfrak{p}_{\eta}}^{\mathfrak{h}_{\eta}}(\tilde{C}) \cong \oplus_{j} A\left[-2 s_{j}\right]\left[\operatorname{dim} \mathfrak{m}_{\eta}-\operatorname{dim} \mathfrak{h}_{\eta}\right]
$$

where $s_{j} \in \mathbf{N}$ are defined as follows. Choose $\phi=(e, h, f) \in J^{H}$ as in 1.2(ii); let $H_{\phi}=\{g \in H ; \operatorname{Ad}(g)(e)=e, \operatorname{Ad}(g)(h)=h, \operatorname{Ad}(g)(f)=f\}$, let $\mathcal{B}$ be the variety of Borel subgroups of $H_{\phi}^{0}$; then $s_{j}$ are defined by $\rho_{\mathcal{B} !} \overline{\mathbf{Q}}_{l}=\oplus_{j} \overline{\mathbf{Q}}_{l}\left[-2 s_{j}\right]$. (See [L4, 11.13].)

1.9. Let $\mathcal{X}$ be the set of all systems $\left(M, M_{0}, \mathfrak{p}, \mathfrak{p}_{*}, \mathfrak{m}, \mathfrak{m}_{*}, \tilde{A}\right)$ where $\mathfrak{p}$ is a parabolic subalgebra of $\mathfrak{h}$ with Levi subalgebra $\mathfrak{m}, \mathfrak{p}=\oplus_{k \in \mathbf{Z}} \mathfrak{p}_{k}, \mathfrak{m}=\oplus_{k \in \mathbf{Z}} \mathfrak{m}_{k}$ where $\mathfrak{p}_{k}=$ $\mathfrak{p} \cap \mathfrak{h}_{k}, \mathfrak{m}_{k}=\mathfrak{m} \cap \mathfrak{h}_{k}, M=e^{\mathfrak{m}}, M_{0}=e^{\mathfrak{m}_{0}}$ and $\tilde{A}$ is a simple perverse sheaf in $\mathcal{Q}\left(\mathfrak{m}_{\eta}\right)$ (up to isomorphism) which is $\eta$-semicuspidal. We have the following result; see [L4, 13.3].

(a) Let $A_{1} \in \mathcal{Q}\left(\mathfrak{h}_{\eta}\right)$. There exists $C_{1}, C_{2}, \ldots, C_{t}, C_{t+1}, \ldots, C_{t+t^{\prime}}$ in $\mathcal{Q}\left(\mathfrak{h}_{\eta}\right)$ such that

$$
A_{1} \oplus C_{1} \oplus C_{2} \oplus \ldots \oplus C_{t}=C_{t+1} \oplus \ldots \oplus C_{t+t^{\prime}}
$$

and each $C_{j}$ is of the form $\operatorname{ind}_{\mathfrak{p}_{\eta}}^{\mathfrak{h}_{\eta}}(\tilde{A})\left[a_{j}\right]$ for some $\left(M, M_{0}, \mathfrak{p}, \mathfrak{p}_{*}, \mathfrak{m}, \mathfrak{m}_{*}, \tilde{A}\right) \in \mathcal{X}$ (depending on $j$ ) and some $a_{j} \in \mathbf{Z}$.

Erratum to [L4. In the definition of a good object in the second paragraph of [L4 13.2], one should insert the words "shifts of" after "direct sum of" (twice).

1.10. Let $s \in \mathbf{Z}-\{0\}$. We show:

(a) the subspace $\mathfrak{h}^{(1)}:=\oplus_{k \in s} \mathbf{z} \mathfrak{h}_{k}$ of $\mathfrak{h}$ is the Lie algebra of a well-defined connected reductive subgroup $H^{(1)}$ of $H$.

We can assume that $s>0$. We shall define $e \in Z_{\geq 0}$ as follows: if $p=0$ we have $e=0$; if $p>0$ we define $e$ by $s=s^{\prime} p^{e}$, where $s^{\prime} \in \mathbf{Z}_{>0}$ is not divisible by $p$. We shall argue by induction on $e$. (When $p=0$ we only have to consider the case $e=0$.) Assume first that $e=0$.

Let $\bar{H}$ be the adjoint group of $H$ and let $\overline{\mathfrak{h}}$ be its Lie algebra. Then $\overline{\mathfrak{h}}$ inherits a Z-grading $\overline{\mathfrak{h}}=\oplus_{k} \overline{\mathfrak{h}}_{k}$ from $\mathfrak{h}$. If we assume known that $\overline{\mathfrak{h}}^{(1)}:=\oplus_{k \in s \mathbf{Z}} \overline{\mathfrak{h}}_{k}$ is the Lie algebra of a well-defined connected reductive subgroup $\bar{H}^{(1)}$ of $\bar{H}$, then we can take $H^{(1)}$ to be the identity component of the inverse image of $\bar{H}^{(1)}$ under the obvious map $H \rightarrow \bar{H}$. Thus we can assume that $H$ is adjoint. Let $\lambda \in Y_{H}$ be such that ${ }_{k}^{\lambda} \mathfrak{h}=\mathfrak{h}_{k}$ for all $k$. Let $\zeta^{\prime}$ be a primitive $s$-th root of 1 in $\mathbf{k}$. (Note that if $p>0$, $s=s^{\prime}$ is not divisible by $p$.) We define $\omega: H \rightarrow H$ by $\omega(g)=\operatorname{Ad}\left(\lambda\left(\zeta^{\prime}\right)\right)(g)$; this is an automorphism of $H$. The automorphism $\omega^{\prime}: \mathfrak{h} \rightarrow \mathfrak{h}$ induced by $\omega$ sends $x \in \mathfrak{h}_{k}$ (where $k \in \mathbf{Z}$ ) to $\zeta^{\prime k} x$. Hence $\omega^{s}=1$ and $\mathfrak{h}^{(1)}$ is equal to $\{x \in \mathfrak{h} ; \omega(x)=x\}$. Let $H^{(1)}$ be the identity component of $\{g \in H ; \omega(g)=g\}$. This is a connected reductive 
group with Lie algebra $\mathfrak{h}^{(1)}$. Thus (a) is proved in the case $e=0$. We now assume that $e \geq 1$ hence $p>0$. We can find an element $x_{0} \in \mathfrak{h}$ such that $\left[x_{0}, x\right]=k x$ for any $k \in \mathbf{Z}$ and any $x \in \mathfrak{g}_{k}$. (We can take $x_{0}$ in the image of the tangent map of $\lambda: \mathbf{k}^{*} \rightarrow H$.) Let $\tilde{\mathfrak{h}}=\left\{x \in \mathfrak{h} ;\left[x_{0}, x\right]=0\right\}$. We have $\tilde{\mathfrak{h}}=\oplus_{k \in p} \mathbf{z} \mathfrak{h}_{k}$. Let $\tilde{H}$ be the identity component of $\left\{g \in H ; \operatorname{Ad}(g) x_{0}=x_{0}\right\}$. Since $x_{0} \in \mathfrak{h}$ is semisimple, it follows that $\tilde{H}$ is reductive with Lie algebra $\tilde{\mathfrak{h}}$. We define a $\mathbf{Z}$-grading $\tilde{\mathfrak{h}}=\oplus_{k^{\prime} \in \mathbf{Z}} \tilde{\mathfrak{h}}_{k^{\prime}}$ by $\tilde{\mathfrak{h}}_{k^{\prime}}=\mathfrak{h}_{p k^{\prime}}$. By the induction hypothesis applied to $\tilde{H}, \tilde{\mathfrak{h}}$ we see that there is a well-defined connected reductive subgroup $\tilde{H}^{(1)}$ of $\tilde{H}$ whose Lie algebra is $\oplus_{k^{\prime} \in(s / p) \mathbf{z}} \tilde{\mathfrak{h}}_{k^{\prime}}=\oplus_{k^{\prime} \in(s / p) \mathbf{z}} \mathfrak{h}_{p k^{\prime}}=\oplus_{k \in s} \mathbf{z} \mathfrak{h}_{k}=\mathfrak{h}^{(1)}$. We can take $H^{(1)}=\tilde{H}^{(1)}$. This completes the inductive proof.

\section{2. $\mathbf{Z} \mapsto$-GRADINGS AND $\epsilon$-SPIRALS}

In this section we introduce the key notion of this paper, namely a spiral. Spirals are analogues in the $\mathbf{Z} / m$-graded setting of parabolic subalgebras in the ungraded or $\mathbf{Z}$-graded setting. We also attach a canonical spiral to each nilpotent element in $\mathfrak{g}_{\delta}$.

2.1. In the rest of this paper, $m \geq 1, G, \mathfrak{g}=\oplus_{i \in \mathbf{Z} / m} \mathfrak{g}_{i}$ are as in 0.1 and $\zeta, \vartheta, \theta$ are as in 0.5 . Recall that for $i \in \mathbf{Z} / m$ we have $\mathfrak{g}_{i}=\left\{x \in \mathfrak{g} ; \theta(x)=\zeta^{i} x\right\}$ and that $\vartheta: G \rightarrow G$ is the (semisimple) automorphism of $G$ which induces $\theta: \mathfrak{g} \rightarrow \mathfrak{g}$; note that $\theta(\operatorname{Ad}(g) x)=\operatorname{Ad}(\vartheta(g)) \theta(x)$ for all $x \in \mathfrak{g}, g \in G$.

We shall fix $\delta \in \mathbf{Z} / m$.

For any semisimple automorphism $\gamma: G \rightarrow G$, we set $G^{\gamma}=\{g \in G ; \gamma(g)=g\}$. By a theorem of Steinberg [St],

(a) $G^{\gamma}$ is a connected reductive subgroup of $G$.

Now $\mathfrak{g}_{\underline{0}}$ is a Lie subalgebra of $\mathfrak{g}$. Recall that $G_{\underline{0}}=G^{\vartheta}$ and that the Ad-action of $G_{\underline{0}}$ on $\mathfrak{g}$ leaves stable $\mathfrak{g}_{i}$ and its closed subset $\mathfrak{g}_{i}^{n i \bar{l}}:=\mathfrak{g}_{i} \cap \mathfrak{g}^{\text {nil }}$ for any $i \in \mathbf{Z} / \mathrm{m}$.

Let $\mathfrak{G}$ be the set of subgroups of $G$ of the form $G^{\operatorname{Ad}(\tau) \vartheta}$ for some semisimple element of finite order $\tau \in G_{\underline{0}}$; by (a), any group in $\mathfrak{G}$ is a connected reductive subgroup of $G$. For example, we have $G_{\underline{0}} \in \mathfrak{G}$; hence we have $G_{\underline{0}}=e^{\mathfrak{g}_{0}}$.

2.2. Let $\langle\rangle:, \mathfrak{g} \times \mathfrak{g} \rightarrow \mathbf{k}$ be a Killing form; it is nondegenerate and it satisfies $\left\langle\mathfrak{g}_{i}, \mathfrak{g}_{j}\right\rangle=0$ whenever $i+j \neq \underline{0}$ in $\mathbf{Z} / m$. Hence for any $i \in \mathbf{Z} / m,\langle\rangle:, \mathfrak{g}_{i} \times \mathfrak{g}_{-i} \rightarrow \mathbf{k}$ is nondegenerate.

2.3. The Morozov-Jacobson theorem in the $\mathbf{Z} / m$-graded setting. We set $J=J^{G}$; see 1.1. For $x \in \mathfrak{g}^{\text {nil }}$ let $J(x)=\{(e, h, f) \in J ; e=x\}, G(x)=\{g \in$ $G ; \operatorname{Ad}(g) x=x\}$ and let $U=U_{G(x)^{0}}$. Recall the following result of MorozovJacobson and Kostant; see $\mathrm{Ko}$.

(a) We have $J(x) \neq \emptyset$. The $U$-action on $J(x)$ given by

$$
u:(e, h, f) \mapsto u(e, h, f):=(e, \operatorname{Ad}(u) h, \operatorname{Ad}(u) f)
$$

is simply transitive.

Assume now that $x \in \mathfrak{g}_{\delta}^{\text {nil }}$. We set

$$
J_{\delta}(x)=\left\{(e, h, f) \in J(x) ; e=x, h \in \mathfrak{g}_{\underline{0}}, f \in \mathfrak{g}_{-\delta}\right\} .
$$

We show:

(b) We have $J_{\delta}(x) \neq \emptyset$. The $\left(U \cap G_{\underline{0}}\right)$-action on $J_{\delta}(x)$ (restriction of the $U$-action in (a)) is simply transitive. 
If $(e, h, f) \in J(x)$, then $\left(\zeta^{-\delta} e, h, \zeta^{\delta} f\right) \in J_{\delta}\left(\zeta^{-\delta} x\right)$ and

$$
\left(\zeta^{-\delta} \theta(e), \theta(h), \zeta^{\delta} \theta(f)\right) \in J\left(\zeta^{-\delta} \theta(x)\right)=J(x)
$$

(we use that $\theta(e)=\zeta^{\delta} e$ ). Hence $(e, h, f) \mapsto\left(\zeta^{-\delta} \theta(e), \theta(h), \zeta^{\delta} \theta(f)\right)$ is a morphism $\theta^{\prime}: J(x) \rightarrow J(x)$. Next we note that $g \mapsto \vartheta(g)$ defines a homomorphism $G(x) \rightarrow$ $G(x)$. (If $\operatorname{Ad}(g) x=x$, then $\theta(x)=\theta(\operatorname{Ad}(g) x)=\operatorname{Ad}(\vartheta(g)) \theta(x)$. Since $\theta(x)=\zeta^{\delta} x$, we see that $\zeta^{\delta} x=\operatorname{Ad}(\vartheta(g)) \zeta^{\delta} x$ hence $x=\operatorname{Ad}(\vartheta(g)) x$ and $\vartheta(g) \in G(x)$.) This restricts to a homomorphism $\theta^{\prime \prime}: U \rightarrow U$ with fixed point set $U^{\theta^{\prime \prime}}$. For $u \in U$, $(e, h, f) \in J(x)$ we have $\theta^{\prime}(u(e, h, f))=\theta^{\prime \prime}(u) \theta^{\prime}(e, h, f)$. By $(\mathrm{a}), J(x)$ is an affine space. Since $\theta^{\prime m}=1$ and $m$ is invertible in $\mathbf{k}$, the fixed point set $J(x)^{\theta^{\prime}}$ is nonempty. Since the $U$-action on $J(x)$ is simply transitive, it follows that this restricts to a simply transitive action of $U^{\theta^{\prime \prime}}$ on $J(x)^{\theta^{\prime}}$. We have $J(x)^{\theta^{\prime}}=J_{\delta}(x)$ and $U^{\theta^{\prime \prime}}=$ $U \cap G_{\underline{0}}$. We see that (b) holds.

2.4. Let $\lambda \in Y_{G_{\underline{0}}}$ (resp. $\mu \in Y_{G_{0}, \mathbf{Q}}$ ). Since $\lambda$ (resp. $\mu$ ) can be viewed as an element of $Y_{G}$ (resp. $\left.Y_{G}, \mathbf{Q}\right)$, the decomposition $\mathfrak{g}=\oplus_{k \in \mathbf{Z}}\left({ }_{k}^{\lambda} \mathfrak{g}\right)\left(\operatorname{resp} . \quad \mathfrak{g}=\oplus_{\kappa \in \mathbf{Q}}\left({ }_{\kappa}^{\mu} \mathfrak{g}\right)\right)$ is defined as in 1.1. For $i \in \mathbf{Z} / m$ and for $k \in \mathbf{Z}$ (resp. $\kappa \in \mathbf{Q}$ ) we set ${ }_{k}^{\lambda} \mathfrak{g}_{i}={ }_{k}^{\lambda} \mathfrak{g} \cap \mathfrak{g}_{i}$ (resp. $\left.{ }_{\kappa}^{\mu} \mathfrak{g}_{i}={ }_{\kappa}^{\mu} \mathfrak{g} \cap \mathfrak{g}_{i}\right)$; we then have $\mathfrak{g}_{i}=\oplus_{k \in \mathbf{Z}}\left({ }_{k}^{\lambda} \mathfrak{g}_{i}\right)\left(\right.$ resp. $\left.\mathfrak{g}_{i}=\oplus_{\kappa \in \mathbf{Q}}\left({ }_{\kappa}^{\mu} \mathfrak{g}_{i}\right)\right)$ for any $i \in \mathbf{Z} / m$ (we now use that $\lambda \in Y_{G_{0}}\left(\right.$ resp. $\left.\mu \in Y_{G_{\underline{0}}, \mathbf{Q}}\right)$ ).

Let $s \in \mathbf{Z}-\{0\}$. We show:

(a) The subspace $\mathfrak{g}^{(1)}:=\oplus_{k \in s} \mathbf{Z}\left({ }_{k}^{\lambda} \mathfrak{g}_{k / s}\right)$ of $\mathfrak{g}$ is the Lie algebra of a well-defined connected reductive subgroup $G^{(1)}$ of $\bar{G}$.

We apply 1.10 (a) to $H=G, \mathfrak{h}=\mathfrak{g}$ with the $\mathbf{Z}$-grading $\mathfrak{g}=\oplus_{k}\left(\begin{array}{l}\lambda \\ k\end{array}\right)$. We see that there is a well-defined reductive connected subgroup $H^{(1)}$ of $G$ whose Lie algebra is $\mathfrak{h}^{(1)}=\oplus_{k \in s \mathbf{Z}}\left({ }_{k}^{\lambda} \mathfrak{g}\right)$. Note that $H^{(1)}$ contains $\lambda\left(\mathbf{k}^{*}\right)$ and is $\vartheta$-stable. We choose $\zeta^{\prime} \in \mathbf{k}^{*}$ such that $\zeta^{\prime s}=\zeta$. We define $\omega: H^{(1)} \rightarrow H^{(1)}$ by $\omega(h)=\operatorname{Ad}\left(\lambda\left(\zeta^{\prime}\right)\right)^{-1} \vartheta(h)$; this is an automorphism of $H^{(1)}$. The automorphism $\omega^{\prime}: \mathfrak{h}^{(1)} \rightarrow \mathfrak{h}^{(1)}$ induced by $\omega$ sends $x \in{ }_{k}^{\lambda} \mathfrak{g}_{i}$ (where $k \in s \mathbf{Z}, i \in \mathbf{Z} / m$ ) to $\zeta^{\prime-k} \zeta^{i} x=\zeta^{i-k / s} x$. Hence $\omega^{\prime m}=1$ and $\mathfrak{g}^{(1)}$ is equal to $\left\{x \in \mathfrak{h}^{(1)} ; \omega^{\prime}(x)=x\right\}$. Let $G^{(1)}$ be the identity component of $\left\{h \in H^{(1)} ; \omega(h)=h\right\}$. Then $G^{(1)}$ is a connected reductive subgroup of $H^{(1)}$ with Lie algebra $\mathfrak{g}^{(1)}$. This proves (a).

Now ${ }_{0}^{\lambda} \mathfrak{g}_{\underline{0}}$ is a Levi subalgebra of a parabolic subalgebra of $\mathfrak{g}_{\underline{0}}$. Hence $e^{\lambda} \mathfrak{g}_{\underline{0}}$ is a well-defined subgroup of $G_{\underline{0}}$ (a Levi subgroup of a parabolic subgroup of $G_{\underline{0}}$ ). We have

(b) $e^{\lambda} \mathfrak{g}_{\underline{0}} \subset G^{(1)}$.

2.5. The definition of $\epsilon$-spirals. In the rest of this section we fix $\epsilon \in\{1,-1\}$. For any $\mu \in Y_{G_{\underline{0}}, \mathbf{Q}}$ and any $N \in \mathbf{Z}$ we set

$$
\epsilon_{\mathfrak{p}_{N}^{\mu}}^{\mu}=\oplus_{\kappa \in \mathbf{Q} ; \kappa \geq N \epsilon}\left({ }_{\kappa}^{\mu} \mathfrak{g}_{\underline{N}}\right) .
$$

If $r \in \mathbf{Z}_{>0}$ is such that $\lambda:=r \mu \in Y_{G_{0}}$ then we have

$$
\epsilon_{\mathfrak{p}_{N}^{\mu}}^{\mu}=\oplus_{k \in \mathbf{Z} ; k \geq r N \epsilon}\left(\begin{array}{l}
\lambda \\
k
\end{array} \mathfrak{g}_{N}\right) .
$$

A collection $\left\{\mathfrak{p}_{N} ; N \in \mathbf{Z}\right\}$ (or $\mathfrak{p}_{*}$ ) of subspaces of $\mathfrak{g}$ is said to be an $\epsilon$-spiral if there exists $\mu \in Y_{G_{\underline{0}}, \mathbf{Q}}$ such that $\mathfrak{p}_{N}={ }^{\epsilon} \mathfrak{p}_{N}^{\mu}$ for any $N \in \mathbf{Z}$. We then set (for $N \in \mathbf{Z}$ )

$$
\mathfrak{u}_{N}=\left\{x \in \mathfrak{g}_{\underline{N}} ;\left\langle x,{ }^{\epsilon} \mathfrak{p}_{-N}^{\mu}\right\rangle=0\right\}=\oplus_{\kappa \in \mathbf{Q} ; \kappa>N \epsilon}\left({ }_{\kappa}^{\mu} \mathfrak{g}_{\underline{N}}\right) .
$$

We say that $\mathfrak{u}_{*}=\left\{\mathfrak{u}_{N} ; N \in \mathbf{Z}\right\}$ is the nilradical of $\mathfrak{p}_{*}$. 
The following properties of $\mathfrak{p}_{*}, \mathfrak{u}_{*}$ are immediate:

$\ldots \subset \mathfrak{p}_{N} \subset \mathfrak{p}_{N-\epsilon m} \subset \mathfrak{p}_{N-2 \epsilon m} \subset \ldots$ for any $N$

$\mathfrak{p}_{N} \subset \mathfrak{g}_{\underline{N}}$ for any $N ; \mathfrak{p}_{N}=0$ if $N \epsilon \gg 0 ; \mathfrak{p}_{N}=\mathfrak{g}_{\underline{N}}$ if $N \epsilon \ll 0$;

$\left[\mathfrak{p}_{N}, \mathfrak{p}_{N^{\prime}}\right] \subset \mathfrak{p}_{N+N^{\prime}}$ for any $N, N^{\prime}$ in $\mathbf{Z}$;

$\ldots \subset \mathfrak{u}_{N} \subset \mathfrak{u}_{N-\epsilon m} \subset \mathfrak{u}_{N-2 \epsilon m} \subset \ldots$ for any $N$;

$\mathfrak{u}_{N} \subset \mathfrak{p}_{N}$ for any $N ; \mathfrak{u}_{N}=\mathfrak{g}_{N}$ if $N \epsilon \ll 0$;

$\left[\mathfrak{u}_{N}, \mathfrak{p}_{N^{\prime}}\right] \subset \mathfrak{u}_{N+N^{\prime}}$ for any $N, N^{\prime}$ in $\mathbf{Z}$.

For $N \in \mathbf{Z}$ we set $\mathfrak{l}_{N}=\mathfrak{p}_{N} / \mathfrak{u}_{N}$ and $\mathfrak{l}=\oplus_{N \in \mathbf{Z}} \mathfrak{l}_{N}$. We have $\mathfrak{l}_{N}=0$ if $N \gg 0$ or if $N \ll 0$ hence $\operatorname{dim} \mathfrak{l}<\infty$; moreover, [,] $: \mathfrak{p}_{N} \times \mathfrak{p}_{N^{\prime}} \rightarrow \mathfrak{p}_{N+N^{\prime}}$ induces an operation $\mathfrak{l}_{N} \times \mathfrak{l}_{N^{\prime}} \rightarrow \mathfrak{l}_{N+N^{\prime}}$ which defines a Lie algebra structure on $\mathfrak{l}$.

Note that $\mathfrak{p}_{0}$ is a parabolic subagebra of the reductive Lie algebra $\mathfrak{g}_{0}$ and $\mathfrak{u}_{0}=$ $\left\{x \in \mathfrak{g}_{0} ;\left\langle x, \mathfrak{p}_{0}\right\rangle=0\right\}$ is the nilradical of $\mathfrak{p}_{0}$. We set $P_{0}=e^{\mathfrak{p}_{0}} \subset G_{\underline{0}}, U_{0}=e^{\mathfrak{u}_{0}} \subset G_{\underline{0}}$. Then $\bar{P}_{0}$ is a parabolic subgroup of $G_{\underline{0}}$ and $U_{0}=U_{P_{0}}$, so that $L_{0}:=P_{0} / U_{0}$ is a connected reductive group. We note that:

(b) The Ad-action of $P_{0}$ on $\mathfrak{g}$ leaves stable $\mathfrak{p}_{N}$ and $\mathfrak{u}_{N}$ for any $N$.

From (b) we see that for any $N$ there is an induced action of $P_{0}$ on $\mathfrak{l}_{N}=\mathfrak{p}_{N} / \mathfrak{u}_{N}$. We show:

(c) The restriction of this action to $U_{0}$ is trivial.

It is enough to show that the ad-action of $\mathfrak{u}_{0}$ on $\mathfrak{p}_{N} / \mathfrak{u}_{N}$ is zero. This follows from the inclusion $\left[\mathfrak{u}_{0}, \mathfrak{p}_{N}\right] \subset \mathfrak{u}_{N}$ which has been noted earlier.

From (b),(c) we see that for any $N$ there is an induced action of $L_{0}=P_{0} / U_{0}$ on $\mathfrak{l}_{N}=\mathfrak{p}_{N} / \mathfrak{u}_{N}$. We show:

(d) if $x \in \mathfrak{p}_{N}, N \epsilon>0$, then $x \in \mathfrak{g}_{N}^{\text {nil }}$.

It is enough to show that for any $x^{\prime} \in \mathfrak{g}$ we have $\operatorname{ad}(x)^{n}\left(x^{\prime}\right)=0$ for $n \gg 0$. We can assume that $x^{\prime} \in \mathfrak{g}_{i}$ for some $i \in \mathbf{Z} / m$. If $N^{\prime} \in \mathbf{Z}$ satisfies $\underline{N^{\prime}}=i$ and $N^{\prime} \epsilon \ll 0$, then $\mathfrak{p}_{N^{\prime}}=\mathfrak{g}_{i}$; thus we have $x^{\prime} \in \mathfrak{p}_{N^{\prime}}$ for some $N^{\prime}$. We have $\overline{\operatorname{ad}}(x) x^{\prime}=\left[x, x^{\prime}\right] \in \mathfrak{p}_{N+N^{\prime}}$, $\operatorname{ad}(x)^{2}\left(x^{\prime}\right) \in \mathfrak{p}_{2 n+N^{\prime}}$ and, more generally, $\operatorname{ad}(x)^{n}\left(x^{\prime}\right) \in \mathfrak{p}_{n N+N^{\prime}}$ for $n \geq 1$. If $n \gg 0$ we have $n N \epsilon+N^{\prime} \epsilon \gg 0$ hence $\mathfrak{p}_{n N+N^{\prime}}=0$; thus, $\operatorname{ad}(x)^{n}\left(x^{\prime}\right)=0$. This proves (d).

An element $\mu \in Y_{G_{0}, \mathbf{Q}}$ is said to be $p$-regular if $r \mu \in Y_{G_{0}}$ for some $r \in \mathbf{Z}_{>0}$ such that $r \notin p \mathbf{Z}$. (This condition holds automatically if $p=0$.) An $\epsilon$-spiral $\mathfrak{p}_{*}$ is said to be $p$-reqular if $\mathfrak{p}_{*}={ }^{\epsilon} \mathfrak{p}_{*}^{\mu}$ for some $p$-regular $\mu \in Y_{G_{0}, \mathbf{Q}}$.

2.6. Splittings of $\epsilon$-spirals. For $\mu \in Y_{G_{0}, \mathbf{Q}}$ and $N \in \mathbf{Z}$ we set

$$
\epsilon \tilde{\mathfrak{l}}_{N}^{\mu}=\oplus_{\kappa \in \mathbf{Q} ; \kappa=N \epsilon}\left({ }_{\kappa}^{\mu} \mathfrak{g}_{\underline{N}}\right)={ }_{N \epsilon}^{\mu} \mathfrak{g}_{\underline{N}} .
$$

If $r \in \mathbf{Z}_{>0}$ is such that $\lambda:=r \mu \in Y_{G_{\underline{0}}}$, then we have

$$
\tilde{\mathfrak{l}}_{N}^{\mu}={ }_{r N \epsilon}^{\lambda} \mathfrak{g}_{N} .
$$

A splitting of an $\epsilon$-spiral $\mathfrak{p}_{*}$ is a collection $\left\{\tilde{\mathfrak{l}}_{N} ; N \in \mathbf{Z}\right\}$ (or $\tilde{\mathfrak{l}}_{*}$ ) of subspaces of $\mathfrak{g}$ such that for some $\mu \in Y_{G_{0}}, \mathbf{Q}$ we have $\mathfrak{p}_{*}={ }^{\epsilon} \mathfrak{p}_{*}^{\mu}$ and $\tilde{\mathfrak{l}}_{N}={ }^{\epsilon} \tilde{\mathfrak{l}}_{N}^{\mu}$ for any $N \in \mathbf{Z}$. Let $\mathfrak{u}_{*}$ be the nilradical of $\mathfrak{p}_{*}$. From the definitions we see that $\mathfrak{p}_{N}=\mathfrak{u}_{N} \oplus \tilde{\mathfrak{l}}_{N}$ for any $N,\left[\tilde{\mathfrak{l}}_{N}, \tilde{\mathfrak{l}}_{N^{\prime}}\right] \subset \tilde{\mathfrak{l}}_{N+N^{\prime}}$ for any $N, N^{\prime}$ and the sum $\tilde{\mathfrak{l}}:=\sum_{N \in \mathbf{Z}} \tilde{\mathfrak{l}}_{N}$ (in $\mathfrak{g}$ ) is direct. Now $\tilde{\mathfrak{l}}$ is a Lie subalgebra of $\mathfrak{g}$ which is $\mathbf{Z}$-graded by the subspaces $\tilde{\mathfrak{l}}_{N}$. Note that the isomorphisms $\tilde{\mathfrak{l}}_{N} \stackrel{\sim}{\longrightarrow} \mathfrak{l}_{N}$ (restrictions of the obvious maps $\mathfrak{p}_{N} \rightarrow \mathfrak{l}_{N}$ ) give rise after taking $\oplus_{N}$ to an isomorphism $\tilde{\mathfrak{l}} \stackrel{\sim}{\longrightarrow} \mathfrak{l}$ which is compatible with the Lie algebra structures and the $\mathbf{Z}$-gradings. 
For $\mu$ as above we can find $\lambda \in Y_{G_{\underline{0}}}$ and $r \in \mathbf{Z}_{>0}$ such that $r \mu=\lambda$. Applying 2.4(a) with $s=r \epsilon$ we see that:

(a) There is a well-defined connected reductive subgroup $\tilde{L}$ of $G$ whose Lie algebra is $\tilde{\mathfrak{l}}$. In particular, $\tilde{\mathfrak{l}}$ and $\mathfrak{l}$ are reductive Lie algebras.

Let $\tilde{L}_{0}=e^{\tilde{\mathfrak{I}}_{0}}$. From 2.4(b) we have:

(b) $\tilde{L}_{0} \subset \tilde{L}$.

We show:

(c) Assume that we have $\tilde{\mathfrak{l}}_{*}=\tilde{}^{\epsilon} \mathfrak{l}_{*}^{\mu}, \mathfrak{p}_{*}={ }^{\epsilon} \mathfrak{p}_{*}^{\mu}$ where $\mu$ is p-regular, that is, $\mu=r \lambda$ with $\lambda \in Y_{G_{0}}$ and $r \in \mathbf{Z}_{>0}$ such that $r \notin p \mathbf{Z}$. Then there exists $\zeta^{\prime}$, a root of 1 in $\mathbf{k}^{*}$ such that $\left.\tilde{\mathfrak{l}}=\left\{x \in \mathfrak{g} ; \operatorname{Ad}\left(\lambda\left(\zeta^{\prime}\right)^{-1}\right) \theta(x)\right)=x\right\}, \tilde{L}=G^{\operatorname{Ad}\left(\lambda\left(\zeta^{\prime}\right)^{-1}\right) \vartheta}=e^{\tilde{\mathfrak{l}}} \subset G$; note that $\tilde{L} \in \mathfrak{G}$.

Let $\zeta^{\prime}$ be a primitive root of 1 of order $r m$ in $\mathbf{k}^{*}$ such that $\zeta^{\prime r \epsilon}=\zeta$. We have $\mathfrak{g}=\oplus_{k \in \mathbf{Z}, i \in \mathbf{Z} / m}\left({ }_{k}^{\lambda} \mathfrak{g}_{i}\right), \tilde{\mathfrak{l}}_{N}={ }_{N r \epsilon}^{\lambda} \mathfrak{g}_{N}$ for all $N \in \mathbf{Z}$. For $k, N^{\prime} \in \mathbf{Z}$ and $x \in{ }_{k}^{\lambda} \mathfrak{g}_{N^{\prime}}$ we have

$$
\operatorname{Ad}\left(\lambda\left(\zeta^{\prime}\right)^{-1}\right)(\theta(x))=\zeta^{\prime-k} \zeta^{N^{\prime}} x=\zeta^{\prime r N^{\prime} \epsilon-k} x .
$$

The condition that $\zeta^{\prime r N^{\prime} \epsilon-k}=1$ is that $r N^{\prime} \epsilon-k \in r m \mathbf{Z}$ or that $k \in r \mathbf{Z}$ and $\underline{N^{\prime}}=k /(r \epsilon)$. We see that

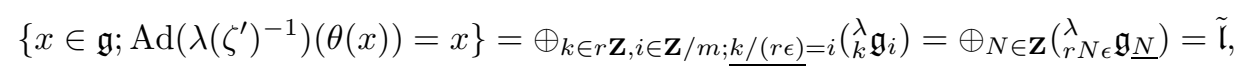

and (c) follows.

We return to the general case.

We have $\lambda\left(\mathbf{k}^{*}\right) \subset \tilde{L}_{0}$; moreover, $\operatorname{Ad}(\lambda(t))$ acts as identity on $\tilde{\mathfrak{l}}_{0}={ }_{0}^{\lambda} \mathfrak{g}_{0}=\mathfrak{L} \tilde{L}_{0}$; thus, $\lambda\left(\mathbf{k}^{*}\right) \subset \mathcal{Z}_{\tilde{L}_{0}}$. Since $\mathbf{k}^{*}$ is connected, we deduce:

(d) $\lambda\left(\mathbf{k}^{*}\right) \subset \mathcal{Z}_{\tilde{L}_{0}}^{0}$.

Note that:

(e) For $t \in \mathbf{k}^{*}, N \in \mathbf{Z}, \operatorname{Ad}(\lambda(t))$ acts on $\mathfrak{l}_{N}$ as $t^{r N \epsilon}$ times identity.

We show:

(f) If $\tilde{\mathfrak{l}}_{*}$ is a splitting of an $\epsilon$-spiral $\mathfrak{p}_{*}$, then $\tilde{\mathfrak{l}}_{*}$ is a splitting of an $(-\epsilon)$-spiral.

Let $\mu \in Y_{G_{0}, \mathbf{Q}}$ be such that $\tilde{\mathfrak{l}}_{*}={ }^{\epsilon} \tilde{\mathfrak{l}}_{*}^{\mu}, \mathfrak{p}_{*}={ }^{\epsilon} \mathfrak{p}_{*}^{\mu}$. Let $\mu^{\prime}=(-1) \mu \in Y_{G_{0}}, \mathbf{Q}$. Then $\tilde{\mathfrak{l}}_{*}={ }^{-\epsilon} \tilde{\mathfrak{l}}_{*}^{\mu^{\prime}}$ is a splitting of the $(-\epsilon)$-spiral ${ }^{-\epsilon} \mathfrak{p}_{*}^{\mu^{\prime}}$.

2.7. Let $\mathfrak{S}$ be the set of splittings of an $\epsilon$-spiral $\mathfrak{p}_{*}$. Clearly, $\mathfrak{S} \neq \emptyset$. Let $U_{0}$ be as in 2.5. Now $U_{0}$ acts on $\mathfrak{S}$ by $u: \tilde{\mathfrak{l}}_{*} \mapsto\left\{\operatorname{Ad}(u) \tilde{\mathfrak{l}}_{N} ; N \in \mathbf{Z}\right\}$. (We use that $\operatorname{Ad}(u) \mathfrak{p}_{N}=\mathfrak{p}_{N}$ for any $N$.) We show:

(a) This $U_{0}$-action on $\mathfrak{S}$ is simply transitive.

Let $\mathfrak{u}_{*}$ be the nilradical of $\mathfrak{p}_{*}$. Let $\tilde{\mathfrak{l}}_{*} \in \mathfrak{S}, \tilde{\mathfrak{l}}_{*}^{\prime} \in \mathfrak{S}$. Since $\tilde{\mathfrak{l}}_{0}, \tilde{\mathfrak{l}}_{0}^{\prime}$ are Levi subalgebras of $\mathfrak{p}_{0}$, there is a unique $u \in U_{0}$ such that $\operatorname{Ad}(u) \tilde{\mathfrak{l}}_{0}=\tilde{\mathfrak{l}}_{0}^{\prime}$. It remains to show that this $u$ satisfies $\operatorname{Ad}(u) \tilde{\mathfrak{l}}_{N}=\tilde{\mathfrak{l}}_{N}^{\prime}$ for any $N$. Let $\tilde{\mathfrak{l}}=\oplus_{N} \tilde{\mathfrak{l}}_{N}, \tilde{\mathfrak{l}}^{\prime}=\oplus_{N} \tilde{\mathfrak{l}}_{N}^{\prime}$ (these are Lie subalgebras of $\mathfrak{g}$ ) and let $\tilde{L}=e^{\tilde{\mathfrak{l}}} \subset G, \tilde{L}^{\prime}=e^{\tilde{\mathfrak{l}}^{\prime}} \subset G$. Let $\mu, \mu^{\prime}$ in $Y_{G_{0}}, \mathbf{Q}$ be such that $\mathfrak{p}_{*}={ }^{\epsilon} \mathfrak{p}_{*}^{\mu}={ }^{\epsilon} \mathfrak{p}_{*}^{\mu^{\prime}}, \tilde{\mathfrak{l}}_{*}=\epsilon \tilde{\mathfrak{l}}_{*}^{\mu}, \tilde{\mathfrak{l}}_{*}^{\prime}=\epsilon \tilde{\mathfrak{l}}_{*}^{\mu^{\prime}}$. We can find $r \in \mathbf{Z}_{>0}$ such that $\lambda:=r \mu \in Y_{G_{\underline{0}}}$, $\lambda^{\prime}:=r \mu^{\prime} \in Y_{G_{\underline{0}}}$. Let $\tilde{L}_{0}$ be as in 2.6 and let $\tilde{L}_{0}^{\prime}$ be the analogous subgroup of $\tilde{L}^{\prime}$. We now fix $N \in \mathbf{Z}$. The Ad-action of $\tilde{L}_{0}$ (resp. $\tilde{L}_{0}^{\prime}$ ) on $\mathfrak{g}$ leaves stable $\tilde{\mathfrak{l}}_{N}, \mathfrak{u}_{N}$ (resp. $\left.\tilde{\mathfrak{l}}_{N}^{\prime}, \mathfrak{u}_{N}\right)$. Let $\tilde{L}_{0}^{\prime \prime}=u \tilde{L}_{0} u^{-1}, \tilde{\mathfrak{l}}_{N}^{\prime \prime}=\operatorname{Ad}(u) \tilde{\mathfrak{l}}_{N} ;$ then the Ad-action of $\tilde{L}_{0}^{\prime \prime}$ on $\mathfrak{g}$ leaves stable $\tilde{\mathfrak{l}}_{N}^{\prime \prime}, \mathfrak{u}_{N}$. Since $\operatorname{Ad}(u) \tilde{\mathfrak{l}}_{0}=\tilde{\mathfrak{l}}_{0}^{\prime}$, we have $u \tilde{L}_{0} u^{-1}=\tilde{L}_{0}^{\prime}$ hence $\tilde{L}_{0}^{\prime}=\tilde{L}_{0}^{\prime \prime}$. Let $T$ be a maximal torus of $\tilde{L}_{0}^{\prime}=\tilde{L}_{0}^{\prime \prime}$. Now the Ad-action of $T$ on $\mathfrak{g}$ leaves stable 
$\tilde{\mathfrak{l}}_{N}^{\prime}, \tilde{\mathfrak{l}}_{N}^{\prime \prime}, \mathfrak{u}_{N}, \mathfrak{p}_{N}$. Let $\mathcal{X}=\operatorname{Hom}\left(T, \mathbf{k}^{*}\right)$. For any $\alpha \in \mathcal{X}$ let

$$
\begin{gathered}
\mathfrak{p}_{N, \alpha}=\left\{x \in \mathfrak{p}_{N} ; \operatorname{Ad}(\tau) x=\alpha(\tau) x \quad \forall \tau \in T\right\}, \quad \mathfrak{u}_{N, \alpha}=\mathfrak{u}_{N} \cap \mathfrak{p}_{N, \alpha}, \\
\tilde{\mathfrak{l}}_{N, \alpha}^{\prime}=\tilde{\mathfrak{l}}_{N}^{\prime} \cap \mathfrak{p}_{N, \alpha}, \quad \tilde{\mathfrak{l}}_{N, \alpha}^{\prime \prime}=\tilde{\mathfrak{l}}_{N}^{\prime \prime} \cap \mathfrak{p}_{N, \alpha} .
\end{gathered}
$$

We have $\tilde{\mathfrak{l}}_{N}^{\prime}=\oplus_{\alpha \in \mathcal{X}} \tilde{\mathfrak{l}}_{N, \alpha}^{\prime}, \tilde{\mathfrak{l}}_{N}^{\prime \prime}=\oplus_{\alpha \in \mathcal{X}} \tilde{\mathfrak{l}}_{N, \alpha}^{\prime \prime}, \mathfrak{u}_{N}=\oplus_{\alpha \in \mathcal{X} \mathfrak{u}_{N, \alpha}}$. Let $\mathcal{R}^{\prime}=\{\alpha \in$ $\left.\mathcal{X} ; \tilde{\mathfrak{l}}_{N, \alpha}^{\prime} \neq 0\right\}, \mathcal{R}^{\prime \prime}=\left\{\alpha \in \mathcal{X} ; \tilde{\mathfrak{l}}_{N, \alpha}^{\prime \prime} \neq 0\right\}, \tilde{\mathcal{R}}=\left\{\alpha \in \mathcal{X} ; \mathfrak{u}_{N, \alpha} \neq 0\right\}$. Since $\tilde{\mathfrak{l}}_{N}^{\prime}, \tilde{\mathfrak{l}}_{N}^{\prime \prime}$ are $T$-stable complements of $\mathfrak{u}_{N}$ in $\mathfrak{p}_{N}$, the $T$-modules $\tilde{\mathfrak{l}}_{N}^{\prime}, \tilde{\mathfrak{l}}_{N}^{\prime \prime}$ are isomorphic, hence $\mathcal{R}^{\prime}=\mathcal{R}^{\prime \prime}$. Since $\lambda^{\prime}\left(\mathbf{k}^{*}\right) \subset \mathcal{Z}_{\tilde{L}_{0}^{\prime}}^{0}($ see $2.6(\mathrm{~d}))$, we have $\lambda^{\prime}\left(\mathbf{k}^{*}\right) \subset T$; hence for any $\alpha \in \mathcal{X}$ we can define $\alpha \bullet \lambda^{\prime} \in \mathbf{Z}$ by $\alpha\left(\lambda^{\prime}(t)\right)=t^{\alpha \bullet \lambda^{\prime}}$ for all $t \in \mathbf{k}^{*}$.

Assume that $\alpha \in \tilde{\mathcal{R}}$. Then for any $t \in \mathbf{k}^{*}, \operatorname{Ad}\left(\lambda^{\prime}(t)\right)$ acts on $\mathfrak{u}_{N, \alpha}$ as multiplication by $t^{\alpha \bullet \lambda^{\prime}}$ hence $\mathfrak{u}_{N, \alpha} \subset \underset{\alpha \bullet \lambda^{\prime}}{\lambda^{\prime}} \mathfrak{g}_{\underline{N}}$; thus ${ }_{\alpha \bullet \lambda^{\prime}}^{\lambda^{\prime}} \mathfrak{g}_{\underline{N}}$ has a nonzero intersection with $\mathfrak{u}_{N}$, so that $\alpha \bullet \lambda^{\prime}>r N \epsilon$. We see that $\tilde{\mathcal{R}} \subset\left\{\alpha \in \mathcal{X} ; \alpha \bullet \lambda^{\prime}>r N \epsilon\right\}$. Assume now that $\alpha \in \mathcal{R}^{\prime}$. Then for any $t \in \mathbf{k}^{*}, \operatorname{Ad}\left(\lambda^{\prime}(t)\right)$ acts on $\mathfrak{l}_{N, \alpha}^{\prime}$ as multiplication by $t^{\alpha \bullet \lambda^{\prime}}$ hence $\mathfrak{l}_{N, \alpha}^{\prime} \subset \underset{\alpha \bullet \lambda^{\prime}}{\lambda^{\prime}} \mathfrak{g}_{N}$; thus, ${ }_{\alpha \bullet \lambda^{\prime}}^{\lambda^{\prime}} \mathfrak{g}_{\underline{N}}$ has a nonzero intersection with $\tilde{\mathfrak{l}}_{N}^{\prime}$, so that $\alpha \bullet \lambda^{\prime}=r N \epsilon$. We see that $\mathcal{R}^{\prime} \subset\left\{\alpha \in \overline{\mathcal{X}} ; \alpha \bullet \lambda^{\prime}=r N \epsilon\right\}$. It follows that $\mathcal{R}^{\prime} \cap \tilde{\mathcal{R}}=\emptyset$ so that $\mathfrak{p}_{N, \alpha}=\tilde{\mathfrak{l}}_{N, \alpha}^{\prime}$ for $\alpha \in \mathcal{R}^{\prime}$. Since $\mathcal{R}^{\prime}=\mathcal{R}^{\prime \prime}$, we have also $\mathcal{R}^{\prime \prime} \cap \tilde{\mathcal{R}}=\emptyset$, so that $\mathfrak{p}_{N, \alpha}=\tilde{\mathfrak{l}}_{N, \alpha}^{\prime \prime}$ for $\alpha \in \mathcal{R}^{\prime \prime}=\mathcal{R}^{\prime}$. Thus, for $\alpha \in \mathcal{R}^{\prime}=\mathcal{R}^{\prime \prime}$ we have $\tilde{\mathfrak{l}}_{N, \alpha}^{\prime}=\tilde{\mathfrak{l}}_{N, \alpha}^{\prime \prime}$ hence $\tilde{\mathfrak{l}}_{N}^{\prime}=\tilde{\mathfrak{l}}_{N}^{\prime \prime}$ and $\tilde{\mathfrak{l}}_{N}^{\prime}=\operatorname{Ad}(u) \tilde{\mathfrak{l}}_{N}$. This proves (a).

For any splitting $\tilde{\mathfrak{l}}_{*}$ of $\mathfrak{p}_{*}$ we denote by $\tilde{L}\left(\tilde{\mathfrak{l}}_{*}\right)$ the connected reductive subgroup $\tilde{L}$ of $G$ associated to $\tilde{\mathfrak{l}}_{*}$ in 2.6. The family of groups $\left(\tilde{L}\left(\tilde{\mathfrak{l}}_{*}\right)\right)$ indexed by the various splittings $\tilde{\mathfrak{l}}_{*}$ of $\mathfrak{p}_{*}$ has the property that any two groups in the family are canonically isomorphic to each other; the isomorphism is provided by conjugation by a welldefined $u \in U_{0}$ (this follows from (a)). It follows that the groups in the family can be identified with a single connected reductive group $L$ which is canonically isomorphic to each group in the family. Note that $L$ is canonically attached to the $\epsilon$-spiral $\mathfrak{p}_{*}$ and that $\mathfrak{L} L=\mathfrak{l}$ canonically. Note also that $L_{0}$ in 2.5 is naturally a closed subgroup of $L$.

2.8. Subspirals coming from parabolics of $\mathfrak{l}_{*}$. Let $\mathfrak{p}_{*}$ be an $\epsilon$-spiral. We define $\mathfrak{u}_{*}, \mathfrak{l}_{*}, \mathfrak{l}$ in terms of $\mathfrak{p}_{*}$ as in 2.5. Let $\mathfrak{q}$ be a parabolic subalgebra of $\mathfrak{l}$ compatible with the Z-grading of $\mathfrak{l}$ that is, such that $\mathfrak{q}=\oplus_{N \in \mathbf{Z}} \mathfrak{q}_{N}$ where $\mathfrak{q}_{N}=\mathfrak{q} \cap \mathfrak{l}_{N}$. For any $N \in \mathbf{Z}$ let $\hat{\mathfrak{p}}_{N}$ be the inverse image of $\mathfrak{q}_{N}$ under the obvious map $\mathfrak{p}_{N} \rightarrow \mathfrak{l}_{N}$. We show:

(a) $\hat{\mathfrak{p}}_{*}$ is an $\epsilon$-spiral. Moreover, if $\mathfrak{p}_{*}$ is p-reqular then $\hat{\mathfrak{p}}_{*}$ is p-regular.

We can find $\mu \in Y_{G_{\underline{0}}}, \mathbf{Q}$ such that $\mathfrak{p}_{*}={ }^{\epsilon} \mathfrak{p}_{*}^{\mu}$; let $\tilde{\mathfrak{l}}_{*}={ }^{\epsilon} \tilde{\mathfrak{l}}_{*}^{\mu}$. Let $\tilde{L}$ be as in 2.6. Let $\tilde{\mathfrak{q}}$ be the Lie subalgebra of $\tilde{\mathfrak{l}}$ corresponding to $\mathfrak{q}$ under the obvious isomorphism $\tilde{\mathfrak{l}} \stackrel{\sim}{\longrightarrow} \mathfrak{l}$ and let $\tilde{\mathfrak{q}}_{N}=\tilde{\mathfrak{q}} \cap \tilde{\mathfrak{l}}_{N}$ so that $\tilde{\mathfrak{q}}=\oplus_{N \in \mathbf{Z}} \tilde{\mathfrak{q}}_{N}$. We then have $\hat{\mathfrak{p}}_{N}=\mathfrak{u}_{N} \oplus \tilde{\mathfrak{q}}_{N}$ for all $N$. Let $r \in \mathbf{Z}_{>0}$ be such that $\lambda:=r \mu \in Y_{G_{\underline{0}}}$; if $\mathfrak{p}_{*}$ is $p$-regular we assume in addition that $r \notin p \mathbf{Z}$.

From 2.6(e) we see that for $t \in \mathbf{k}^{*}, \operatorname{Ad}(\lambda(t))$ leaves stable each $\tilde{\mathfrak{q}}_{N}$ hence it leaves stable $\tilde{\mathfrak{q}}$. It follows that $\mathbf{k}^{*}$ acts via $t \mapsto \operatorname{Ad}(\lambda(t))$ on the variety of Levi subalgebras of $\tilde{\mathfrak{q}}$; since this variety is isomorphic to an affine space, there exists a Levi subalgebra $\mathfrak{m}$ of $\tilde{\mathfrak{q}}$ such that $\operatorname{Ad}(\lambda(t)) \mathfrak{m}=\mathfrak{m}$ for all $t \in \mathbf{k}^{*}$. Let $R$ be the closed connected subgroup of $\tilde{L}$ (a torus) such that $\mathfrak{L} R$ is the center of $\mathfrak{m}$. Since $\tilde{\mathfrak{q}}$ is a parabolic subalgebra of $\tilde{\mathfrak{l}}$ with Levi subalgebra $\mathfrak{m}$, we can find $\lambda^{\prime} \in Y_{R}$ such that, 
setting for any $N^{\prime} \in \mathbf{Z}$ :

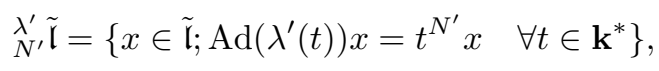

we have $\tilde{\mathfrak{q}}=\oplus_{N^{\prime} \in \mathbf{Z}_{>0}}\left(\stackrel{\lambda^{\prime}}{N^{\prime}} \tilde{\mathfrak{l}}\right), \mathfrak{m}={ }_{0}^{\lambda^{\prime}} \tilde{\mathfrak{l}}$. We have $\mathfrak{m}=\oplus_{N} \mathfrak{m}_{N}$ where $\mathfrak{m}_{N}=\mathfrak{m} \cap \tilde{\mathfrak{l}}_{N}$ and $\mathfrak{m}_{0}$ is a Levi subalgebra of a parabolic subalgebra of $\mathfrak{m}$. Hence a Cartan subalgebra of $\mathfrak{m} \cap \tilde{\mathfrak{l}}_{0}$ is also a Cartan subalgebra of $\mathfrak{m}$, so that it contains the center of $\mathfrak{m}$. Thus the center of $\mathfrak{m}$ is contained in $\tilde{\mathfrak{l}}_{0}$, so that $R \subset \tilde{L}_{0}$. Since for any $t, t^{\prime} \in \mathbf{k}^{*}$, $\lambda(t)$ is contained in $\mathcal{Z}_{\tilde{L}_{0}}$ and $\lambda^{\prime}\left(t^{\prime}\right) \in \tilde{L}_{0}$, we have $\lambda(t) \lambda^{\prime}\left(t^{\prime}\right)=\lambda^{\prime}\left(t^{\prime}\right) \lambda(t)$. We can view $\lambda^{\prime}$ as an element of $Y_{G_{0}}$ hence ${ }_{k}^{\lambda^{\prime}} \mathfrak{g}_{i}$ is defined for $k \in \mathbf{Z}, i \in \mathbf{Z} / m$ and we have

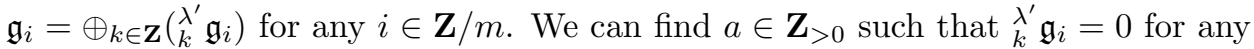
$i \in \mathbf{Z} / m$ and any $k \in \mathbf{Z}-[-a, a]$. Let $b$ be an integer such that $b>2 a, b \notin p \mathbf{Z}$. We define $\lambda^{\prime \prime} \in Y_{G_{\underline{0}}}$ by $\lambda^{\prime \prime}(t)=\lambda\left(t^{b}\right) \lambda^{\prime}(t)=\lambda^{\prime}(t) \lambda\left(t^{b}\right)$ for all $t \in \mathbf{k}^{*}$. By definition, for $k \in \mathbf{Z}, i \in \mathbf{Z} / m$ we have:

$$
\begin{aligned}
{ }_{k}^{\lambda^{\prime \prime}} \mathfrak{g}_{i} & =\left\{x \in \mathfrak{g}_{i} ; \operatorname{Ad}\left(\lambda\left(t^{b}\right) \lambda^{\prime}(t)\right) x=t^{k} x \quad \forall t \in \mathbf{k}^{*}\right\} \\
& =\oplus_{k^{\prime}, k_{2} ; k^{\prime} \in b \mathbf{Z}, k_{2} \in \mathbf{Z}, k^{\prime}+k_{2}=k}\left(\begin{array}{l}
\lambda \\
k^{\prime} / b
\end{array} \mathfrak{g}_{i} \cap \underset{k_{2}}{\lambda^{\prime}} \mathfrak{g}_{i}\right) .
\end{aligned}
$$

When ${ }_{k}^{\lambda^{\prime \prime}} \mathfrak{g}_{i} \neq 0$ then $k=b k_{1}+k_{2}$ for some $k_{1} \in \mathbf{Z} \cap[-a, a], k_{2} \in \mathbf{Z}$; in this case, $k_{1}, k_{2}$ are uniquely determined by $k$ since $b>2 a$. Thus, we have

$$
\begin{gathered}
\stackrel{\lambda_{k}^{\prime \prime}}{{ }^{\prime}} \mathfrak{g}_{i}={ }_{k_{1}}^{\lambda} \mathfrak{g}_{i} \cap \underset{k_{2}}{\lambda^{\prime}} \mathfrak{g}_{i} \text { if } k=b k_{1}+k_{2} \text { with } k_{1}, k_{2} \text { in } \mathbf{Z}, \\
{ }_{k}^{\lambda^{\prime \prime}} \mathfrak{g}_{i}=0 \text {, otherwise. }
\end{gathered}
$$

Let $\mu^{\prime}=\frac{1}{b r} \lambda^{\prime \prime} \in Y_{G_{\underline{0}}, \mathbf{Q}}$ and let $\mathfrak{p}_{*}^{\prime}={ }_{\mathfrak{p}_{*}}^{\mu^{\prime}}$. For $N \in \mathbf{Z}$ we have

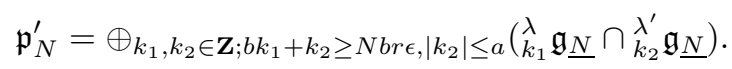

The only integer multiple of $b$ in $[-a, a]$ is 0 ; hence the condition that $k_{2} \geq b(r N \epsilon-$ $k_{1}$ ) (with $k_{2} \in[-a, a]$ ) is equivalent to the condition that either $0>b\left(r N \epsilon-k_{1}\right)$, $k_{2} \in[-a, a]$ or that $0=b\left(r N \epsilon-k_{1}\right), k_{2} \in[0, a]$. Thus, $\mathfrak{p}_{N}^{\prime}=X \oplus X^{\prime}$, where

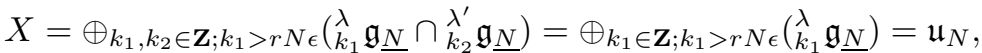

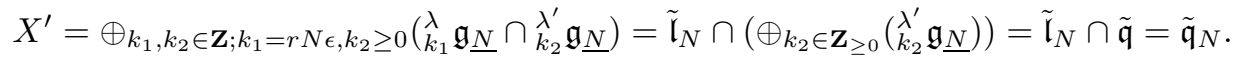

Thus, we have $\mathfrak{p}_{N}^{\prime}=\mathfrak{u}_{N} \oplus \tilde{\mathfrak{q}}_{N}=\hat{\mathfrak{p}}_{N}$. This proves (a).

From the computation in the previous proof we can extract the following:

(b) the splitting $\tilde{\mathfrak{l}}_{*}^{\mu^{\prime}}$ of the $\epsilon$-spiral $\hat{\mathfrak{p}}_{*}={ }_{\mathfrak{p}_{*}}^{\mu^{\prime}}$ is equal to $\mathfrak{m}_{*}$.

2.9. The spiral attached to an element $x \in \mathfrak{g}_{\delta}^{\text {nil }}$. In the remainder of this paper we fix $\eta \in \mathbf{Z}-\{0\}$ such that $\underline{\eta}=\delta$.

In this subsection we assume that $\epsilon=\dot{\eta}$; see 0.12 . Let $x \in \mathfrak{g}_{\delta}^{\text {nil }}$. We associate to $x$ an $\epsilon$-spiral as follows. By 2.3(b), we can find $\phi=(e, h, f) \in J_{\delta}(x)$ such that $e=x$. Let $\iota=\iota_{\phi} \in Y_{G}$ be as in 1.1. Since the differential of $\iota$ is the linear map $\mathbf{k} \rightarrow \mathfrak{g}, z \mapsto z h \in \mathfrak{g}_{0}$, we have $\iota\left(\mathbf{k}^{*}\right) \subset G_{\underline{0}}$ so that $\iota$ can be viewed as an element of $Y_{G_{\underline{0}}}$. Then $\mathfrak{p}_{*}^{\phi}:=\epsilon_{\mathfrak{p}_{*}}^{(|\eta| / 2) \iota}$ is an $\epsilon$-spiral with splitting $\tilde{\mathfrak{l}}_{*}^{\phi}:={ }^{\epsilon} \tilde{\mathfrak{l}}_{*}^{(|\eta| / 2) \iota}$. Note that for $N \in \mathbf{Z}$ we have

$$
\mathfrak{p}_{N}^{\phi}=\oplus_{k \in \mathbf{Z} ; k \geq 2 N \epsilon}\left(\begin{array}{l}
\iota \\
k /|\eta|
\end{array} \mathfrak{g}_{\underline{N}}\right), \quad \tilde{\mathfrak{l}}_{N}^{\phi}={ }_{2 N / \eta}^{\iota} \underline{\mathfrak{g}}_{N} \text { if } 2 N / \eta \in \mathbf{Z}, \quad \tilde{\mathfrak{l}}_{N}^{\phi}=0 \text { if } 2 N / \eta \notin \mathbf{Z} .
$$

We show that:

(a) The $\epsilon$-spiral $\mathfrak{p}_{*}^{\phi}$ is p-regular; it depends only on $x$, not on $\phi$. 
The $p$-regularity follows from the fact that $2 \notin p \mathbf{Z}$. We now prove the second statement of (a). By 2.3(b), another choice for $\phi$ must be of the form $u \phi$ where $u \in U_{G(x)^{0}} \cap G_{\underline{0}}$. Let $\iota^{\prime}=\iota_{u \phi}$. For $t \in \mathbf{k}^{*}$ we have $\iota^{\prime}(t)=u \iota(t) u^{-1}$ hence ${ }_{k}^{\iota^{\prime}} \mathfrak{g}_{i}=\operatorname{Ad}(u)\left({ }_{k}^{\iota} \mathfrak{g}_{i}\right)$ for any $k \in \mathbf{Z}, i \in \mathbf{Z} / m$. It follows that for $N \in \mathbf{Z}$ we have $\mathfrak{p}_{N}^{u \phi}=\operatorname{Ad}(u) \mathfrak{p}_{N}^{\phi}$. To show that $\mathfrak{p}_{N}^{u \phi}=\mathfrak{p}_{N}^{\phi}$, it is enough to show that $\operatorname{Ad}(u) \mathfrak{p}_{N}^{\phi}=\mathfrak{p}_{N}^{\phi}$. It is enough to show:

$\operatorname{Ad}(u)\left({ }_{k}^{\iota} \mathfrak{g}\right) \subset \oplus_{k^{\prime} ; k^{\prime} \geq k}\left(\begin{array}{c}\iota \\ k^{\prime}\end{array}\right)$ gor any $u \in G(x), k \in \mathbf{Z}$.

Let $P$ be the parabolic subgroup of $G$ such that $\mathfrak{L} P=\oplus_{k \in \mathbf{Z} ; k \geq 0}\left({ }_{k}^{\iota} \mathfrak{g}\right)$. Clearly, $\operatorname{Ad}(g)\left({ }_{k}^{\iota} \mathfrak{g}\right) \subset \oplus_{k^{\prime} ; k^{\prime} \geq k}\left({ }_{k^{\prime}} \mathfrak{g}\right)$ for any $g \in P, k \in \mathbf{Z}$. Hence it is enough to note the known inclusion $G(x) \subset P$. This proves (a).

In view of (a) we will write $\mathfrak{p}_{*}^{x}$ instead of $\mathfrak{p}_{*}^{\phi}$, where $\phi$ is any element in $J_{\delta}(x)$; let $\mathfrak{u}_{*}^{x}$ be the nilradical of $\mathfrak{p}_{*}^{x}$. Now the splitting $\tilde{\mathfrak{l}}_{*}^{\phi}$ depends in general on $\phi$. We set $\tilde{\mathfrak{l}}^{\phi}=\oplus_{N \in \mathbf{Z}} \tilde{\mathfrak{l}}_{N}^{\phi}$; this is a $\mathbf{Z}$-graded Lie subalgebra of $\mathfrak{g}$. Let $\tilde{L}^{\phi}=e^{\tilde{\mathfrak{l}}^{\phi}} \subset G$; we have $\tilde{L}^{\phi} \in \mathfrak{G}$. Let $\tilde{L}_{0}^{\phi}=e^{\tilde{\mathfrak{I}}_{0}^{\phi}} \subset \tilde{L}^{\phi}$. We show:

(b) We have $x \in \tilde{\mathfrak{l}}_{\eta}^{\phi}$; more precisely, $x$ belongs to $\stackrel{\stackrel{\circ}{\mathfrak{l}}}{\eta}_{\eta}^{\phi}$ (the open $\tilde{L}_{0}^{\phi}$-orbit on $\tilde{\mathfrak{l}}_{\eta}^{\phi}$ ).

The first statement is the same as $x \in{ }_{2}^{\iota} \mathfrak{g}_{\delta}$; this follows from the equality $[h, x]=$ $2 x$. The second statement can be deduced from [L4, 4.2(a)].

We set $\tilde{L}_{0}^{\phi}(x)=\tilde{L}_{0}^{\phi} \cap G(x), G_{\underline{0}}(x)=G_{\underline{0}} \cap G(x)$. We show:

(c) The inclusion $\tilde{L}_{0}^{\phi}(x) \rightarrow \bar{G}_{\underline{0}}(x)$ induces an isomorphism on the groups of components.

Let $P_{0}$ be the parabolic subgroup of $G_{\underline{0}}$ such that $\mathfrak{L} P_{0}=\mathfrak{p}_{0}^{x}=\oplus_{k \in \mathbf{Z} ; k \geq 0}\left({ }_{k}^{\iota} \mathfrak{g}_{\underline{0}}\right)$ and let $U_{0}=U_{P_{0}}$. We set $P_{0}(x)=P_{0} \cap G(x), U_{0}(x)=U_{0} \cap G(x)$. Then $\tilde{L}_{0}^{\phi}$ is a Levi subgroup of $P_{0}$ so that $P_{0}=\tilde{L}_{0}^{\phi} U_{0}$ (semidirect product) and $P_{0}(x)=\tilde{L}_{0}^{\phi}(x) U_{0}(x)$ (semidirect product). Since $U_{0}(x)$ is a connected unipotent group we see that the inclusion $\tilde{L}_{0}^{\phi}(x) \rightarrow P_{0}(x)$ induces an isomorphism on the groups of components. It remains to show that $P_{0}(x)=G_{\underline{0}}(x)$. As we have noted in the proof of (a), we have $G(x) \subset P$ hence $G_{0}(x) \subset P \cap G_{\underline{0}}$; since $P \cap G_{0}$ and $P_{0}$ have the same Lie algebra, namely $\mathfrak{p}_{0}^{x}$, they must have the same identity component; since $P_{0}$ is parabolic in $G_{\underline{0}}$, we must have $P \cap G_{\underline{0}}=P_{0}$, so that $G_{\underline{0}}(x) \subset P_{0}$ and therefore $G_{\underline{0}}(x) \subset P_{0}(x)$. Since the reverse inclusion is obvious, we see that $P_{0}(x)=G_{\underline{0}}(x)$ and (c) is proved.

We show:

(d) If $g \in G_{\underline{0}}$ is such that $\operatorname{Ad}\left(g^{-1}\right)(x) \in \mathfrak{p}_{\eta}^{x}$, then $g \in P_{0}$.

The assumption of (d) implies that $g \in P$. (We use [L4, 5.7] applied to the trivial $\mathbf{Z}$-grading of $\mathfrak{g}$ that is, the $\mathbf{Z}$-grading such that in [L4, 3.1] we have $\mathfrak{g}_{N}=0$ for $N \neq 0$.) Thus, we have $g \in P \cap G_{\underline{0}}$. As in the proof of (c) we have $P \cap G_{\underline{0}}=P_{0}$ and (d) follows.

We show:

(e) The $P_{0}$-orbit of $x$ in $\mathfrak{p}_{\eta}^{x}$ is open dense in $\mathfrak{p}_{\eta}^{x}$.

We argue as in [L4, 5.9]. It is enough to show that $\operatorname{dim}\left(P_{0}\right)-\operatorname{dim}\left(P_{0} \cap G(x)\right)=$ $\operatorname{dim} \mathfrak{p}_{\eta}^{x}$ or equivalently that

$$
\operatorname{dim} \mathfrak{p}_{0}^{x}-\operatorname{dim} \operatorname{ker}\left(\operatorname{ad}(x): \mathfrak{p}_{0}^{x} \rightarrow \mathfrak{g}_{\delta}\right)=\operatorname{dim} \mathfrak{p}_{\eta}^{x} .
$$

Since $x \in \mathfrak{p}_{\eta}^{x}($ see $(\mathrm{b}))$ and $\left[\mathfrak{p}_{0}^{x}, \mathfrak{p}_{\eta}^{x}\right] \subset \mathfrak{p}_{\eta}^{x}$, we have $\operatorname{ad}(x)\left(\mathfrak{p}_{0}^{x}\right) \subset \mathfrak{p}_{\eta}^{x}$ so that it is enough to show that

$$
\operatorname{dim} \operatorname{ker}\left(\operatorname{ad}(x): \mathfrak{p}_{0}^{x} \rightarrow \mathfrak{p}_{\eta}^{x}\right)=\operatorname{dim} \mathfrak{p}_{0}^{x}-\operatorname{dim} \mathfrak{p}_{\eta}^{x}
$$


or equivalently, that $\operatorname{ad}(x): \mathfrak{p}_{0}^{x} \rightarrow \mathfrak{p}_{\eta}^{x}$ is surjective. By the representation theory of $\mathfrak{s l}_{2}$, the linear map

$$
\operatorname{ad}(x): \oplus_{k \in \mathbf{Z} ; k \geq 0}\left({ }_{k}^{\iota} \mathfrak{g}\right) \rightarrow \oplus_{k \in \mathbf{Z} ; k \geq 2}\left({ }_{k}^{\iota} \mathfrak{g}\right)
$$

is surjective. This restricts for any $i \in \mathbf{Z} / m$ to a (necessarily surjective) map

$$
\operatorname{ad}(x): \oplus_{k \in \mathbf{Z} ; k \geq 0}\left({ }_{k}^{\iota} \mathfrak{g}_{i}\right) \rightarrow \oplus_{k \in \mathbf{Z} ; k \geq 2}\left({ }_{k}^{\iota} \mathfrak{g}_{i+\delta}\right) .
$$

Taking $i=0$ we see that $\operatorname{ad}(x): \mathfrak{p}_{0}^{x} \rightarrow \mathfrak{p}_{\eta}^{x}$ is surjective. This proves (e).

The assignment $x \mapsto \mathfrak{p}_{*}^{x}$ is a $\mathbf{Z} / m$-analogue of an assignment in the case of $\mathbf{Z}$ graded Lie algebras given in [L4, §5] which is in turn modelled on a construction in [KL, 7.1].

\section{Admissible Systems}

In this section we introduce the set $\underline{\mathfrak{T}}_{\eta}$ of $G_{\underline{0}^{-}}$-conjugacy classes of admissible systems, which will be used to index the blocks in $\mathcal{D}_{G_{\underline{0}}}\left(\mathfrak{g}_{\delta}^{\text {nil }}\right)$. We also define a map that assigns a pair $(\mathcal{O}, \mathcal{L})$ (where $\mathcal{O}$ is a $G_{\underline{0}^{-}}$orbit in $\mathfrak{g}_{\delta}^{\text {nil }}$ and $\mathcal{L}$ is an irreducible $G_{\underline{0}}$-equivariant local system on it) an element in $\underline{\mathfrak{T}}_{\eta}$.

3.1. Definition of admissible systems. We preserve the setup of 2.1 .

Let $\mathfrak{T}_{\eta}^{\prime}$ be the set consisting of all systems $\left(M, M_{0}, \mathfrak{m}, \mathfrak{m}_{*}, \tilde{C}\right)$, where $M \in \mathfrak{G}$, $\mathfrak{m}=\mathfrak{L} M, \mathfrak{m}_{*}$ is a $\mathbf{Z}$-grading of $\mathfrak{m}, M_{0}=e^{\mathfrak{m}_{0}} \subset M, \tilde{C}$ is a simple cuspidal $M_{0^{-}}$ equivariant perverse sheaf on $\mathfrak{m}_{\eta}$ (up to isomorphism).

Until the end of 3.4 we fix $\dot{\xi}=\left(M, M_{0}, \mathfrak{m}, \mathfrak{m}_{*}, \tilde{C}\right) \in \mathfrak{T}_{\eta}^{\prime}$. Let $\iota \in Y_{M}$ be associated to $\tilde{C}$ as in $1.2(\mathrm{c})$,(a) (with $M, \tilde{C}$ instead of $H, A$ ), so that ${ }_{k}^{\iota} \mathfrak{m}=\mathfrak{m}_{\eta k / 2}$ for any $k \in \mathbf{Z}$ such that $\eta k / 2 \in \mathbf{Z}$ and ${ }_{k}^{\iota} \mathfrak{m}=0$ for any $k \in \mathbf{Z}$ such that $\eta k / 2 \notin \mathbf{Z}$. Then we have $\mathfrak{m}_{k^{\prime}}={ }_{2 k^{\prime} / \eta} \mathfrak{m}$ for $k^{\prime} \in \mathbf{Z}$ such that $2 k^{\prime} / \eta \in \mathbf{Z}$ and $\mathfrak{m}_{k^{\prime}}=0$ for $k^{\prime} \in \mathbf{Z}$ such that $2 k^{\prime} / \eta \notin \mathbf{Z}$. Note that $\iota\left(\mathbf{k}^{*}\right)$ is contained in $\mathcal{Z}_{M_{0}}^{0}$.

The system $\dot{\xi}$ is said to be admissible if conditions (a),(b) below are satisfied:

(a) we have $\mathfrak{m}_{N} \subset \mathfrak{g}_{N}$ for any $N \in \mathbf{Z}$;

(b) there exists an element $\tau$ of finite order in the torus $\iota\left(\mathbf{k}^{*}\right) \mathcal{Z}_{M}^{0}$ of $M_{0}$ such that $M=G^{\operatorname{Ad}(\tau) \vartheta}$.

We now consider the following condition on $\dot{\xi}$ which may or may not hold.

(c) $\mathfrak{m}_{*}$ is a splitting of some p-regular 1-spiral or, equivalently (see 2.6(f)), of some p-regular (-1)-spiral.

The following result will be proved in 3.2-3.4.

(d) $\dot{\xi}$ is admissible if and only if $\dot{\xi}$ satisfies (c).

We now make some comments on the significance of condition (b). Assume that condition (a) is satisfied and that $\tau$ is any semisimple element of finite order of $G_{\underline{0}}$ such that $M=G^{\operatorname{Ad}(\tau) \vartheta}$. We show that we have automatically

$$
\tau \in \iota\left(\mathbf{k}^{*}\right) \mathcal{Z}_{M}
$$

Note that $\vartheta(\tau)=\tau$ since $\tau \in G_{0}$ hence $\tau \in G^{\operatorname{Ad}(\tau) \vartheta}=M$.

Let $N \in \mathbf{Z}$ be such that $2 N / \eta \in \mathbf{Z}$. Since $\mathfrak{m}_{N} \subset \mathfrak{g}_{N}, \theta$ acts on $\mathfrak{m}_{N}$ as $\zeta^{N}$; since $\operatorname{Ad}(\tau) \theta$ acts as 1 on $\mathfrak{m}$ we see that $\operatorname{Ad}(\tau)$ acts on $\mathfrak{m}_{N}$ as $\zeta^{-N}$. On the other hand, for $t \in \mathbf{k}^{*}, \operatorname{Ad}(\iota(t))$ acts on $\mathfrak{m}_{N}$ as $t^{2 N / \eta}$. Hence if $t_{0} \in \mathbf{k}^{*}$ satisfies $t_{0}^{2 / \eta}=\zeta^{-1}$, then we have $\operatorname{Ad}\left(\iota\left(t_{0}\right)\right) \operatorname{Ad}\left(\tau^{-1}\right)=t_{0}^{2 N / \eta} \zeta^{N}=\zeta^{-N} \zeta^{N}=1$ on $\mathfrak{m}_{N}$. It follows that 
$\operatorname{Ad}\left(\iota\left(t_{0}\right)\right) \operatorname{Ad}\left(\tau^{-1}\right)=1$ on $\mathfrak{m}$. Since $\iota\left(t_{0}\right) \tau^{-1} \in M$, we deduce that $\iota\left(t_{0}\right) \tau^{-1} \in \mathcal{Z}_{M}$ hence $\tau \in \iota\left(\mathbf{k}^{*}\right) \mathcal{Z}_{M}$, as asserted.

We see that condition (b) is a strengthening of (e) in which $\tau$ is required to lie not only in $\iota\left(\mathbf{k}^{*}\right) \mathcal{Z}_{M}$ but in its identity component.

\subsection{We show:}

(a) For any element $\tau_{0}$ of finite order in a torus $T$ there exists $\lambda_{0} \in Y_{T}$ such that $\tau_{0} \in \lambda_{0}\left(\mathbf{k}^{*}\right)$.

We can find $c \in \mathbf{Z}_{>0}$ such that $c \notin p \mathbf{Z}$ and $\tau_{0}^{c}=1$. Let $\mu_{c}=\left\{z \in \mathbf{k}^{*} ; z^{c}=1\right\}$. For some $a \in \mathbf{N}$ we can identify $T=\left(\mathbf{k}^{*}\right)^{a}$ and $\tau_{0}$ with $\left(z_{1}, \ldots, z_{a}\right) \in\left(\mu_{c}\right)^{a} \subset T$. Now $\mu_{c}$ is cyclic with generator $z_{0}$. Thus we have $z_{1}=z_{0}^{k_{1}}, \ldots, z_{a}=z_{0}^{k_{a}}$, where $k_{1}, \ldots, k_{a}$ are integers. We define $\lambda_{0} \in Y_{T}$ by $t \mapsto\left(t^{k_{1}}, \ldots, t^{k_{a}}\right)$. Then $\tau_{0}=\lambda_{0}\left(z_{0}\right)$, as desired.

We remark that in the proof of (a) we can assume that:

(b) $k_{1} \in \mathbf{Z}_{>0}, k_{1} \notin p \mathbf{Z}$.

Indeed, if $p=0$, then $k_{1} \notin p \mathbf{Z}$ is automatic. Assume now that $p>0$. We write $k_{1}=k_{1}^{\prime} p^{e}$, where $k_{1}^{\prime} \in \mathbf{Z}-p \mathbf{Z}, e \in \mathbf{Z}_{\geq 0}$. Define $z_{0}^{\prime} \in \mu_{c}$ by $z_{0}^{\prime}=z_{0}^{p^{e}}$. This is again a generator of $\mu_{c}$. (Recall that $c \notin p \mathbf{Z}$.) We have $z_{1}=\left(z_{0}^{\prime}\right)^{k_{1}^{\prime}}, z_{j}=\left(z_{0}^{\prime}\right)^{k_{j}^{\prime}}$, where $k_{j}^{\prime} \in \mathbf{Z}_{>0}$ for $j=2,3, \ldots, a$. Thus we can replace $z_{0}, k_{1}, \ldots, k_{s}$ by $z_{0}^{\prime}, k_{1}^{\prime}, \ldots, k_{s}^{\prime}$, where $k_{1}^{\prime} \in \mathbf{Z}_{>0}, k_{1}^{\prime} \notin p \mathbf{Z}$. This proves (b).

We now assume that $\tau$ as in 3.1(b) is given. We show:

(c) There exist $f \in \mathbf{Z}_{>0}$ and $\lambda^{\prime} \in Y_{\mathcal{Z}_{M}^{0}}$ such that $f \notin p \mathbf{Z}$ and such that, if $\lambda \in Y_{\iota\left(\mathbf{k}^{*}\right) \mathcal{Z}_{M}^{0}}$ is defined by $\lambda(t)=\iota\left(t^{f}\right) \lambda^{\prime}(t)$ for all $t$, then $\tau \in \lambda\left(\mathbf{k}^{*}\right)$.

If $\iota$ is identically 1 , then (c) follows from (a) applied to $T=\mathcal{Z}_{M}^{0}$ (we can take $f=1$ ). Assume now that $\iota$ is not identically 1 . Then $\iota: \mathbf{k}^{*} \rightarrow M$ has finite kernel. Let $T=\mathbf{k}^{*} \times \mathcal{Z}_{M}^{0}$; we define $d: T \rightarrow \iota\left(k^{*}\right) \mathcal{Z}_{M}^{0}$ by $d(t, g)=\iota(t) g$. By definition, $\iota\left(k^{*}\right)$ is contained in the derived subgroup of $M$ hence it has finite intersection with $\mathcal{Z}_{M}^{0}$. It follows that $d$ has finite kernel. It is also surjective, hence we can find $\tilde{\tau} \in T$ of finite order such that $d(\tilde{\tau})=\tau$. Using (a), we can find $\lambda_{0} \in Y_{T}$ such that $\tilde{\tau} \in \lambda_{0}\left(\mathbf{k}^{*}\right)$; moreover, by (b), we can assume that, setting $\lambda_{0}(t)=\left(\lambda_{1}(t), \lambda^{\prime}(t)\right)$ with $\lambda_{1} \in Y_{\mathbf{k}^{*}}, \lambda^{\prime} \in Y_{\mathcal{Z}_{M}^{0}}$, we have $\lambda_{1}(t)=t^{f}$ for all $t$ where $f \in \mathbf{Z}_{>0}, f \notin p \mathbf{Z}$. Let $\lambda=d \lambda_{0}: \mathbf{k}^{*} \rightarrow \iota\left(k^{*}\right) \mathcal{Z}_{M}^{0}$. We have $\lambda(t)=\iota\left(\lambda_{1}(t)\right) \lambda^{\prime}(t)=\iota\left(t^{f}\right) \lambda^{\prime}(t)$ for $t \in \mathbf{k}^{*}$. Since $d(\tilde{\tau})=\tau$ and $\tilde{\tau} \in \lambda_{0}\left(\mathbf{k}^{*}\right)$, we have $\tau \in \lambda\left(\mathbf{k}^{*}\right)$. This proves (c).

3.3. We now assume that $\tau$ as in 3.1(b) is given; let $\lambda, \lambda^{\prime}, f$ be as in 3.2(c). We assume also that 3.1(a) holds. We can find $c \in \mathbf{k}^{*}$ of finite order such that $\lambda(c)=\tau$. (If $\tau \neq 1$, then $\lambda$ is not identically 1 so it has finite kernel and any $c \in \lambda^{-1}(\tau)$ has finite order; if $\tau=1$ we can take $c=1$.)

Since $\lambda\left(\mathbf{k}^{*}\right) \subset M_{0}$ and $M_{0} \subset G_{0}$ (as a consequence of our assumption 3.1(a)), we can view $\lambda$ as an element of $Y_{G_{0}}$ hence ${ }_{k}^{\lambda} \mathfrak{g}_{i}$ is defined for any $k \in \mathbf{Z}, i \in \mathbf{Z} / \mathrm{m}$. Since $\lambda\left(\mathbf{k}^{*}\right) \subset M$, we can view $\lambda$ as an element of $Y_{M}$ hence ${ }_{k}^{\lambda} \mathfrak{m}$ is defined for any $k \in \mathbf{Z}$.

For $t \in \mathbf{k}^{*}, k \in \mathbf{Z}$ such that $2 k / \eta \in \mathbf{Z}$ and $x \in \mathfrak{m}_{k}$ we have $\operatorname{Ad}(\lambda(t)) x=$ $\operatorname{Ad}\left(\iota\left(t^{f}\right)\right) \operatorname{Ad}\left(\lambda^{\prime}(t)\right) x=\operatorname{Ad}\left(\iota\left(t^{f}\right)\right) x=t^{2 k f / \eta} x$ (we use that $\lambda^{\prime}(t) \in \mathcal{Z}_{M}^{0}$ ). Thus $\mathfrak{m}_{k} \subset{ }_{2 k f / \eta}^{\lambda} \mathfrak{m}$. Recall also that $\mathfrak{m}_{k} \neq 0$ implies $k / \eta \in \mathbf{Z}$; see $1.2(\mathrm{e})$. Since the subspaces $\mathfrak{m}_{k}$ form a direct sum decomposition of $\mathfrak{m}$ and the subspaces ${ }_{j}^{\lambda} \mathfrak{m}$ form a 
direct sum decomposition of $\mathfrak{m}$, it follows that:

$$
\begin{aligned}
& \mathfrak{m}_{k}={ }_{2 k f / \eta}^{\lambda} \mathfrak{m} \text { for any } k \in \eta \mathbf{Z} \text { and } \\
& { }_{j}^{\lambda} \mathfrak{m}=0 \text { unless } j=2 k f / \eta \quad \text { for some } k \in \eta \mathbf{Z} .
\end{aligned}
$$

For $k \in \mathbf{Z}, i \in \mathbf{Z} / m$ and $x \in{ }_{k}^{\lambda} \mathfrak{g}_{i}$ we have

$$
\operatorname{Ad}(\tau) \theta(x)=\operatorname{Ad}(\lambda(c)) \theta(x)=\zeta^{i} \operatorname{Ad}(\lambda(c)) x=\zeta^{i} c^{k} x .
$$

Since $\mathfrak{m}=\{x \in \mathfrak{g} ; \operatorname{Ad}(\tau)(\theta(x))=x\}$, we see that:

$$
\mathfrak{m}=\oplus_{j \in \mathbf{Z}, i \in \mathbf{Z} / m ; \zeta^{i} c^{j}=1}\left({ }_{j}^{\lambda} \mathfrak{g}_{i}\right) .
$$

If ${ }_{j}^{\lambda} \mathfrak{g}_{i}$ is nonzero and contained in $\mathfrak{m}$ then ${ }_{j}^{\lambda} \mathfrak{m}$ is nonzero hence by (a) we have $j=2 f k / \eta$ for some $k \in \mathbf{Z}$ and $\mathfrak{m}_{k}$ is a nonzero subspace of $\mathfrak{g}_{i}$; thus, by 3.1(a), we have $i=\underline{k}$ and $2 k / \eta \in \mathbf{Z}$. Thus we can rewrite (b) as follows:

$$
\mathfrak{m}=\oplus_{k \in \eta \mathbf{Z} ; \zeta^{k} c^{2 f k / \eta}=1}\left(\begin{array}{l}
\lambda \\
2 f k / \eta
\end{array} \mathfrak{g}_{k}\right),
$$

that is,

$$
\mathfrak{m}=\oplus_{k \in \eta \mathbf{Z} ;\left(\zeta^{\eta} c^{2 f}\right)^{k / \eta}=1}\left(\begin{array}{l}
\lambda \\
2 f k / \eta \\
\mathfrak{g}_{k}
\end{array}\right) .
$$

Assume now that $\mathfrak{m}_{\eta} \neq 0$. Using (a) we have $\mathfrak{m}_{\eta}={ }_{2 f}^{\lambda} \mathfrak{m} \neq 0$. By 3.1(a) we have $\mathfrak{m}_{\eta} \subset \mathfrak{g}_{\delta}$. It follows that $\mathfrak{m}$ has nonzero intersection with ${ }_{2 f}^{\lambda} \mathfrak{g}_{\delta}$. Now $\operatorname{Ad}(\tau) \theta$ acts on ${ }_{2 f}^{\lambda} \mathfrak{g}_{\delta}$ as multiplication by $\zeta^{\eta} c^{2 f}$ and it acts on $\mathfrak{m}$ as the identity. It follows that $\zeta^{\eta} c^{2 f}=1$. Thus (c) can be rewritten as:

$$
\mathfrak{m}=\oplus_{k \in \eta \mathbf{Z}}\left(\begin{array}{l}
\lambda \\
2 f k / \eta \\
\mathfrak{g}_{k}
\end{array}\right) .
$$

Next we assume that $\mathfrak{m}_{\eta}=0$. By the definition of $\iota$ (see 3.1) this implies that $\iota$ is identically 1 hence $\mathfrak{m}=\mathfrak{m}_{0}$. From (a) we see that $\mathfrak{m}={ }_{0}^{\lambda} \mathfrak{m}$, hence in (c) all summands corresponding to $k \neq 0$ are zero. Thus (d) remains true in this case. We see also that

$$
\mathfrak{m}_{k}={ }_{2 f k \epsilon}^{|\eta| \lambda} \mathfrak{g}_{\underline{k}}
$$

for all $k \in \mathbf{Z}$. Setting $\mu=|\eta| \lambda /(2 f)$ we see that $\mathfrak{m}_{*}$ is a splitting of the $p$-regular $\epsilon$-spiral ${ }_{\mathfrak{p}_{*}^{\frac{1}{2 f}}|\eta| \lambda}$. We see that if $\dot{\xi}$ is admissible then it satisfies 3.1(c).

3.4. Assume now that $\dot{\xi}$ satisfies 3.1(c). Thus $\mathfrak{m}_{*}$ is a splitting of an $\epsilon$-spiral $\mathfrak{p}_{*}={ }^{\epsilon} \mathfrak{p}_{*}^{\mu}$ where $\mu$ is $p$-regular. Applying the conjugacy result 2.7(a) to the two splittings $\mathfrak{m}_{*},{ }^{\epsilon} \tilde{\mathfrak{l}}_{*}^{\mu}$ we see that there exists a $p$-regular $\mu^{\prime}$ such that $\mathfrak{p}_{*}={ }^{\epsilon} \mathfrak{p}_{*}^{\mu^{\prime}}, \mathfrak{m}_{*}={ }^{\epsilon \tilde{\mathfrak{l}}_{*}^{\mu^{\prime}}}$. Thus we can find $\lambda \in Y_{G_{\underline{0}}}, r \in \mathbf{Z}_{>0}$ such that $r \notin p \mathbf{Z}$ and

$$
\mathfrak{m}_{N}={ }_{r N \epsilon}^{\lambda} \mathfrak{g}_{\underline{N}}
$$

for any $N \in \mathbf{Z}$. In particular, 3.1(a) holds. We now show that 3.1(b) holds. From 2.6(c) we see that $M=G^{\operatorname{Ad}\left(\lambda\left(\zeta^{\prime}\right)^{-1}\right) \vartheta}$ for some root of unity $\zeta^{\prime} \in \mathbf{k}^{*}$. Let $\tau=\lambda\left(\zeta^{\prime}\right)^{-1}$. It remains to show that $\lambda\left(\zeta^{\prime}\right)^{-1} \in \iota\left(\mathbf{k}^{*}\right) \mathcal{Z}_{M}^{0}$. More generally, we show that $\lambda(t) \in \iota\left(\mathbf{k}^{*}\right) \mathcal{Z}_{M}^{0}$ for any $t \in \mathbf{k}^{*}$. Now $\lambda$ can be viewed as an element of $Y_{M}$ hence ${ }_{k}^{\lambda} \mathfrak{m}$ is well-defined for any $k \in \mathbf{Z}$ and we have ${ }_{r N \epsilon}^{\lambda} \mathfrak{m}=\mathfrak{m}_{N}$ for any $N \in \mathbf{Z}$. Recall that for $N \in \mathbf{Z}$ we have $\mathfrak{m}_{N}={ }_{2 N / \eta}^{\iota} \mathfrak{m}$ if $N / \eta \in \mathbf{Z}$ and $\mathfrak{m}_{N}=0$ if $N / \eta \notin \mathbf{Z}$. We see that for any $N \in \eta \mathbf{Z}$ and any $t \in \mathbf{k}^{*}, \operatorname{Ad}(\lambda(t))$ acts on $\mathfrak{m}_{N}$ as $t^{r N \epsilon}$ while $\operatorname{Ad}\left(\iota\left(t^{|\eta|}\right)\right)$ acts on $\mathfrak{m}_{N}$ as $t^{2 N \epsilon}$. Hence $\operatorname{Ad}\left(\lambda(t)^{2} \iota(t)^{-r|\eta|}\right)$ acts on $\mathfrak{m}_{N}$ as 1 . Since $\mathfrak{m}$ is the sum of the subspaces $\mathfrak{m}_{N}$, we see that $\operatorname{Ad}\left(\lambda(t)^{2} \iota(t)^{-r|\eta|}\right)$ acts on $\mathfrak{m}$ as 1 . It follows that $\lambda(t)^{2} \iota(t)^{-r|\eta|} \in \mathcal{Z}_{M}$. Since $t \mapsto \lambda(t)^{2} \iota(t)^{-r|\eta|}$ is a homomorphism of 
the connected group $\mathbf{k}^{*}$ into $\mathcal{Z}_{M}$, its image must be contained in $\mathcal{Z}_{M}^{0}$. Thus, for any $t \in \mathbf{k}^{*}$ we have $\lambda(t)^{2} \iota(t)^{-r|\eta|} \in \mathcal{Z}_{M}^{0}$ hence $\lambda\left(t^{2}\right) \in \iota\left(\mathbf{k}^{*}\right) \mathcal{Z}_{M}^{0}$. Since any $t^{\prime} \in \mathbf{k}^{*}$ is a square, it follows that $\lambda\left(t^{\prime}\right) \in \iota\left(\mathbf{k}^{*}\right) \mathcal{Z}_{M}^{0}$ for any $t^{\prime} \in \mathbf{k}^{*}$. We see that, if $\dot{\xi}$ satisfies 3.1(c), then $\dot{\xi}$ is admissible. This completes the proof of 3.1(d).

3.5. The map $\Psi: \mathcal{I}\left(\mathfrak{g}_{\delta}\right) \rightarrow \underline{\mathfrak{I}}_{\eta}$. Let $\mathcal{I}\left(\mathfrak{g}_{\delta}\right)$ be the set of pairs $(\mathcal{O}, \mathcal{L})$ where $\mathcal{O}$ is a $G_{\underline{0}}$-orbit on $\mathfrak{g}_{\delta}^{\text {nil }}$ and $\mathcal{L}$ is an irreducible $G_{\underline{0}}$-equivariant local system on $\mathcal{O}$ defined up to isomorphism. Since $G_{\underline{0}}$ acts on $\mathfrak{g}_{\delta}^{n i l}$ with finitely many orbits, see [Vi], the set $\mathcal{I}\left(\mathfrak{g}_{\delta}\right)$ is finite.

Let $\mathfrak{T}_{\eta}$ be the set of all $\left(M, M_{0}, \mathfrak{m}, \mathfrak{m}_{*}, \tilde{C}\right) \in \mathfrak{T}_{\eta}^{\prime}$ which are admissible (see 3.1$)$ or equivalently (see $3.1(\mathrm{~d})$ ) are such that $\mathfrak{m}_{*}$ is a splitting of some $p$-regular $\epsilon$-spiral. The group $G_{\underline{0}}$ acts in an obvious way by conjugation on $\mathfrak{T}_{\eta}$; we denote by $\underline{\mathfrak{T}}_{\eta}$ the set of orbits, which is a finite set. We will define a map $\Psi: \mathcal{I}\left(\mathfrak{g}_{\delta}\right) \rightarrow \underline{\mathfrak{T}}_{\eta}$. Let $(\mathcal{O}, \mathcal{L}) \in \mathcal{I}\left(\mathfrak{g}_{\delta}\right)$. Choose $x \in \mathcal{O}$ and $\phi \in J_{\delta}(x) ;$ define $\mathfrak{u}_{*}^{x}, \tilde{\mathfrak{l}}_{*}^{\phi}, \tilde{\mathfrak{l}}^{\phi}, \tilde{L}^{\phi}, \tilde{L}_{0}^{\phi}$ as in 2.9 . Recall that $\tilde{L}^{\phi} \in \mathfrak{G}$. We have $x \in{\stackrel{\circ}{\tilde{l}_{\eta}}}_{\eta}($ see $2.9(\mathrm{~b}))$. By $2.9(\mathrm{c}), \mathcal{L}_{1}:=\mathcal{L}_{\tilde{\mathfrak{l}}_{\eta} \phi}$ is an irreducible $\tilde{L}_{0}^{\phi}$-equivariant local system on ${\stackrel{\circ}{\tilde{l}_{\eta}}}_{\eta}$. Let $A$ be the simple $\tilde{L}_{0}^{\phi}$-equivariant perverse sheaf on $\tilde{\mathfrak{l}}_{\eta}^{\phi}$ whose restriction to $\stackrel{\circ}{\mathfrak{l}}_{\eta}^{\phi}$ is $\mathcal{L}_{1}\left[\operatorname{dim} \tilde{\mathfrak{l}}_{\eta}^{\phi}\right]$. The map $1.5(\mathrm{~b})$ associates to $A$ an element $\left(M, M_{0}, \mathfrak{m}, \mathfrak{m}_{*}, \tilde{C}\right)$ of $\mathfrak{M}_{\eta}\left(\tilde{L}^{\phi}\right)$ well defined up to conjugation by $\tilde{L}_{0}^{\phi}$. Using 1.6(a) we can find a parabolic subalgebra $\mathfrak{q}$ of $\tilde{\mathfrak{l}}^{\phi}$ compatible with the $\mathbf{Z}$ grading of $\tilde{\mathfrak{l}}^{\phi}$ and such that $\mathfrak{m}$ is a Levi subalgebra of $\mathfrak{q}$. Setting $\mathfrak{p}_{N}^{\prime}=\mathfrak{u}_{N}^{\phi}+\mathfrak{q}_{N}$ for any $N \in \mathbf{Z}$, we see from $2.8\left(\right.$ a) that $\mathfrak{p}_{*}^{\prime}$ is a $p$-regular $\epsilon$-spiral and from $2.8(\mathrm{~b})$ that $\mathfrak{m}_{*}$ is a splitting of $\mathfrak{p}_{*}^{\prime}$. We see that $\left(M, M_{0}, \mathfrak{m}, \mathfrak{m}_{*}, \tilde{C}\right) \in \mathfrak{T}_{\eta}$.

We now show that the $G_{\underline{0}^{-}}$orbit of $\left(M, M_{0}, \mathfrak{m}, \mathfrak{m}_{*}, \tilde{C}\right)$ is independent of the choices made. First, if $x, \phi$ are already chosen, then the $\tilde{L}_{0}^{\phi}$-orbit of $\left(M, M_{0}, \mathfrak{m}, \mathfrak{m}_{*}, \tilde{C}\right)$ is well defined hence the $G_{\underline{0}}$-orbit of $\left(M, M_{0}, \mathfrak{m}, \mathfrak{m}_{*}, \tilde{C}\right)$ is well defined (since $\tilde{L}_{0}^{\phi} \subset G_{\underline{0}}$ ). The independence of the choice of $\phi$ (when $x$ is given) follows from the homogeneity of $J_{\delta}(x)$ under the group $U \cap G_{0}$ in 2.3(b). Finally, the independence of the choice of $x$ follows from the homogeneity of $\mathcal{O}$ under the group $G_{\underline{0}}$. Thus,

$$
(\mathcal{O}, \mathcal{L}) \mapsto\left(G_{\underline{0}}-\text { orbit of }\left(M, M_{0}, \mathfrak{m}, \mathfrak{m}_{*}, \tilde{C}\right)\right)
$$

is a well-defined map $\Psi: \mathcal{I}\left(\mathfrak{g}_{\delta}\right) \rightarrow \underline{\mathfrak{T}}_{\eta}$.

3.6. Let $\dot{\xi}=\left(M, M_{0}, \mathfrak{m}, \mathfrak{m}_{*}, \tilde{C}\right) \in \mathfrak{T}_{\eta}$. Let $\mathcal{O}_{\dot{\xi}}$ be the unique $G_{\underline{0}^{-}}$orbit in $\mathfrak{g}_{\delta}^{\text {nil }}$ that contains $\stackrel{\circ}{\mathfrak{m}_{\eta}}$. Let $\dot{\xi}^{\prime}=\left(M^{\prime}, M_{0}^{\prime}, \mathfrak{m}^{\prime}, \mathfrak{m}_{*}^{\prime}, \tilde{C}^{\prime}\right) \in \mathfrak{T}_{\eta}$. We show:

(a) If $\mathcal{O}_{\dot{\xi}}=\mathcal{O}_{\dot{\xi}^{\prime}}$, then there exists $g \in G_{\underline{0}}$ such that $\operatorname{Ad}(g)$ carries $\left(M, M_{0}, \mathfrak{m}, \mathfrak{m}_{*}\right)$ to $\left(M^{\prime}, M_{0}^{\prime}, \mathfrak{m}^{\prime}, \mathfrak{m}_{*}^{\prime}\right)$.

By [L4, 3.3], we can find $\phi=(e, h, f) \in J^{M}, \phi^{\prime}=\left(e^{\prime}, h^{\prime}, f^{\prime}\right) \in J^{M^{\prime}}$ such that:

(b) $e \in \stackrel{\circ}{\mathfrak{m}}_{\eta}, h \in \mathfrak{m}_{0}, f \in \mathfrak{m}_{-\eta}, e^{\prime} \in \stackrel{\circ}{\prime}_{\eta}^{\prime}, h^{\prime} \in \mathfrak{m}_{0}^{\prime}, f^{\prime} \in \mathfrak{m}_{-\eta}^{\prime}$.

We set $\iota=\iota_{\phi} \in Y_{M}, \iota^{\prime}=\iota_{\phi^{\prime}} \in Y_{M^{\prime}}$. By 1.2(a),(c),(e), we have

$$
\mathfrak{m}_{k}={ }_{2 k / \eta}^{\iota} \mathfrak{m}, \quad \mathfrak{m}_{k}^{\prime}={ }_{2 k / \eta}^{\iota^{\prime}} \mathfrak{m}^{\prime} \text { if } k \in \eta \mathbf{Z}, \mathfrak{m}_{k}=\mathfrak{m}_{k}^{\prime}=0 \text { if } k \in \mathbf{Z}-\eta \mathbf{Z} .
$$

By assumption, we have $e^{\prime}=\operatorname{Ad}\left(g_{1}\right) e$ for some $g_{1} \in G_{\underline{0}}$. Replacing the system $\left(M, M_{0}, \mathfrak{m}, \mathfrak{m}_{*}, \tilde{C}, \phi\right)$ by its image under $\operatorname{Ad}\left(g_{1}\right)$, we see that we can assume that $e=e^{\prime}$. Using $3.1\left(\right.$ a) for $\dot{\xi}$ and $\dot{\xi}^{\prime}$, we can view $\phi, \phi^{\prime}$ as elements of $J_{\delta}^{G}$ with the 
same first component. By 2.3(b), we can find $g_{2} \in G_{\underline{0}}$ such that $\operatorname{Ad}\left(g_{2}\right)$ carries $\phi$ to $\phi^{\prime}$. Replacing $\left(M, M_{0}, \mathfrak{m}, \mathfrak{m}_{*}, \tilde{C}, \phi\right)$ by its image under $\operatorname{Ad}\left(g_{1}\right)$, we see that we can assume that $\phi=\phi^{\prime}$ as elements of $J^{G}$. It follows that $\iota=\iota^{\prime}$ as elements of $Y_{G}$.

Let

$$
G_{\phi}=\{g \in G ; \operatorname{Ad}(g)(e)=e, \operatorname{Ad}(g)(h)=h, \operatorname{Ad}(g)(f)=f\} .
$$

Since $e, h, f$ are contained in $\mathfrak{m}$ we have $\mathcal{Z}_{M} \subset G_{\phi}$. Similarly, since $e, h, f$ are contained in $\mathfrak{m}^{\prime}$, we have $\mathcal{Z}_{M^{\prime}} \subset G_{\phi}$. We have also $\mathcal{Z}_{M}^{0} \subset G_{0}$ (since the center of $\mathfrak{m}$ is contained in $\left.\mathfrak{m}_{0} \subset \mathfrak{g}_{\underline{0}}\right)$; similarly we have $\mathcal{Z}_{M^{\prime}}^{0} \subset G_{\underline{0}}$. Thus, $\mathcal{Z}_{M}^{0}$ and $\mathcal{Z}_{M^{\prime}}^{0}$ are tori in $\left(G_{\phi} \cap G_{\underline{0}}\right)^{0}$. We show that $\mathcal{Z}_{M}^{0}$ is a maximal torus of $\left(G_{\phi} \cap G_{\underline{0}}\right)^{0}$. Indeed, assume that $S$ is a torus of $\left(G_{\phi} \cap G_{0}\right)^{0}$ that contains $\mathcal{Z}_{M}^{0}$. Since $S \subset G_{\phi}$, for any $s \in S$ we have $\operatorname{Ad}(s) h=h$ hence $s \iota(t)=\iota(t) s$, that is, $\operatorname{Ad}(\iota(t)) s=s$ for $t \in \mathbf{k}^{*}$. Since $S$ contains $\mathcal{Z}_{M}^{0}$, for any $s \in S, z \in \mathcal{Z}_{M}^{0}$ we have $\operatorname{Ad}(z) s=s$. Since $S \subset G_{\underline{0}}$ we have $\vartheta(s)=s$ for any $s \in S$. We see that $\operatorname{Ad}(\iota(t)) \operatorname{Ad}(z) \vartheta(s)=s$ for any $t \in \mathbf{k}^{*}$, $z \in \mathcal{Z}_{M}^{0}, s \in S$. We can find $\tau \in \iota\left(\mathbf{k}^{*}\right) \mathcal{Z}_{M}^{0}$ such that $M=G^{\operatorname{Ad}(\tau) \vartheta}$. We have seen that $\operatorname{Ad}(\tau) \vartheta(s)=s$ for $s \in S$. Thus $S \subset M$. Since $S \subset G_{\phi}$, we have

$$
S \subset M_{\phi}:=\{g \in M ; \operatorname{Ad}(g)(e)=e, \operatorname{Ad}(g)(h)=h, \operatorname{Ad}(g)(f)=f\},
$$

hence $S \subset M_{\phi}^{0}$. Since $e$ is a distinguished nilpotent element of $\mathfrak{m}$, we have $M_{\phi}^{0}=$ $\mathcal{Z}_{M}^{0}$. Thus we have $S \subset \mathcal{Z}_{M}^{0}$. By assumption, we have $\mathcal{Z}_{M}^{0} \subset S$, hence $\mathcal{Z}_{M}^{0}=S$. Thus $\mathcal{Z}_{M}^{0}$ is indeed a maximal torus of $\left(G_{\phi} \cap G_{\underline{0}}\right)^{0}$, as claimed. Similarly we see that $\mathcal{Z}_{M^{\prime}}^{0}$ is a maximal torus of $\left(G_{\phi} \cap G_{\underline{0}}\right)^{0}$. Since any two maximal tori of $\left(G_{\phi} \cap G_{0}\right)^{0}$ are conjugate, we can find $g_{3}$ in $\left(G_{\phi} \cap G_{\underline{0}}\right)^{0}$ such that $\operatorname{Ad}\left(g_{3}\right)$ carries $\mathcal{Z}_{M}^{0}$ to $\mathcal{Z}_{M^{\prime}}^{0}$. (It also carries $\phi$ to $\phi$.)

Replacing $\left(M, M_{0}, \mathfrak{m}, \mathfrak{m}_{*}, \tilde{C}, \phi\right)$ by its image under $\operatorname{Ad}\left(g_{3}\right)$, we see that we can assume that $\mathcal{Z}_{M}^{0}=\mathcal{Z}_{M^{\prime}}^{0}$ and $\phi=\phi^{\prime}$.

Assume now that $e=0$ so that $e^{\prime}=0$. By the definition of $\iota=\iota^{\prime}$ we see that $\iota=\iota^{\prime}$ is identically 1 hence $\mathfrak{m}=\mathfrak{m}_{0}, \mathfrak{m}^{\prime}=\mathfrak{m}_{0}^{\prime}$ and $G_{\phi}=G$. Since $e=0$ is distinguished in $\mathfrak{m}$ it follows that $M$ is a torus. Hence $M=\mathcal{Z}_{M}^{0}$. Similarly $M^{\prime}=\mathcal{Z}_{M^{\prime}}^{0}$. Since $\mathcal{Z}_{M}^{0}=\mathcal{Z}_{M^{\prime}}^{0}$ it follows that $M=M^{\prime}$. We see that (a) holds in this case.

In the remainder of the proof we assume that $e \neq 0$ hence $e^{\prime} \neq 0$. Recall that $M=G^{\operatorname{Ad}(\iota(t)) \operatorname{Ad}(z) \vartheta}, M^{\prime}=G^{\operatorname{Ad}\left(\iota\left(t^{\prime}\right)\right) \operatorname{Ad}\left(z^{\prime}\right) \vartheta}$, for some $t, t^{\prime}$ in $\mathbf{k}^{*}$ and some $z, z^{\prime}$ in $\mathcal{Z}_{M}^{0}=\mathcal{Z}_{M^{\prime}}^{0}$. Since $e \in \mathfrak{m}_{\eta}$, we have $\operatorname{Ad}(\iota(t)) \operatorname{Ad}(z) \theta(e)=e ;$ since $\operatorname{Ad}(z)$ acts as 1 on $\mathfrak{m}$, we deduce that $t^{2} \zeta^{\eta} e=e$ and since $e \neq 0$, we see that $t^{2}=\zeta^{-\eta}$. Similarly, since $e \in \mathfrak{m}_{\eta}^{\prime}$ we have $\operatorname{Ad}\left(\iota\left(t^{\prime}\right)\right) \operatorname{Ad}\left(z^{\prime}\right) \theta(e)=e$ and $t^{\prime 2}=\zeta^{-\eta}$.

We show that for any $k \in \mathbf{Z}$ we have $\mathfrak{m}_{k} \subset \mathfrak{m}^{\prime}$. By 1.2(e) we can assume that $k \in \eta \mathbf{Z}$. Let $x \in \mathfrak{m}_{k}$. We must show that $\operatorname{Ad}\left(\iota\left(t^{\prime}\right)\right) \operatorname{Ad}\left(z^{\prime}\right) \theta(x)=x$. Since $\operatorname{Ad}\left(z^{\prime}\right)$ acts by 1 on $\mathfrak{m}$, it is enough to show that $\zeta^{k} t^{\prime 2 k / \eta} x=x$ or that $\left(\zeta^{\eta} t^{\prime 2}\right)^{k / \eta} x=x$. This follows from $t^{\prime 2}=\zeta^{-\eta}$.

Thus we have $\mathfrak{m}_{k} \subset \mathfrak{m}^{\prime}$. Since this holds for any $k \in \mathbf{Z}$, we deduce that $\mathfrak{m} \subset \mathfrak{m}^{\prime}$. Interchanging the roles of $\mathfrak{m}, \mathfrak{m}^{\prime}$ we see that $\mathfrak{m}^{\prime} \subset \mathfrak{m}$ hence $\mathfrak{m}=\mathfrak{m}^{\prime}$. This implies that $M=M^{\prime}$. Since $\iota=\iota^{\prime}$, we see from (c) that $\mathfrak{m}_{*}=\mathfrak{m}_{*}^{\prime}$. From $\mathfrak{m}_{0}=\mathfrak{m}_{0}^{\prime}$ we deduce that $M_{0}=M_{0}^{\prime}$. This completes the proof of (a).

The following result can be extracted from the proof of (a).

(d) If $\mathfrak{m}_{\eta}=0$ (so that $e=0$ ), then $\mathfrak{m}=\mathfrak{m}_{0}$ is a Cartan subalgebra of $\mathfrak{g}_{0}$. 
3.7. Let $\left(M, M_{0}, \mathfrak{m}, \mathfrak{m}_{*}, \tilde{C}\right) \in \mathfrak{T}_{\eta}$. Let $x \in \stackrel{\circ}{\mathfrak{m}}_{\eta}$. We choose $\phi=(e, h, f) \in J^{M}$ such that $e=x, h \in \mathfrak{m}_{0}, f \in \mathfrak{m}_{-\eta}$ (see [L4, 3.3]). We can view $x$ as an element of $\mathfrak{g}_{\delta}^{\text {nil }}$ and $\phi$ as an element of $J_{\delta}(x)$. We define $\tilde{\mathfrak{l}}_{*}=\tilde{\mathfrak{l}}_{*}^{\phi}$ as in 2.9. Recall that for $N \in \mathbf{Z}$ we have:

$$
\tilde{\mathfrak{l}}_{N}={ }_{2 N / \eta}^{\iota} \mathfrak{g}_{\underline{N}} \text { if } 2 N / \eta \in \mathbf{Z}, \quad \tilde{\mathfrak{l}}_{N}=0 \text { if } 2 N / \eta \notin \mathbf{Z},
$$

where $\iota=\iota_{\phi} \in Y_{G}$. Let $\tilde{\mathfrak{l}}=\oplus_{N} \tilde{\mathfrak{l}}_{N} \subset \mathfrak{g}$ and let $\tilde{L}=e^{\tilde{\mathfrak{l}}} \subset G$. We show:

(a) $\mathfrak{m}$ is a Levi subalgebra of a parabolic subalgebra of $\tilde{\mathfrak{l}}$ which is compatible with the $\mathbf{Z}$-grading of $\tilde{\mathfrak{l}}$.

We shall prove (a) without the statement of compatibility with the $\mathbf{Z}$-grading; then the full statement of (a) would follow from 1.6(a).

Assume first that $x=0$. Then $h=0$ hence $\iota$ is the constant map with image 1. It follows that $\tilde{\mathfrak{l}}=\tilde{\mathfrak{l}}_{0}=\mathfrak{g}_{0}$ and $\mathfrak{m}=\mathfrak{m}_{0} ;$ moreover: by $3.6(\mathrm{~d}), \mathfrak{m}$ is a Cartan subalgebra of $\mathfrak{g}_{0}$. Hence in this case (a) is immediate. In the rest of the proof we assume that $x \neq 0$.

Since $\stackrel{\circ}{\mathfrak{m}}_{\eta}$ carries a cuspidal local system, for any $N \in \mathbf{Z}$ such that $2 N / \eta \in \mathbf{Z}$ we have $\mathfrak{m}_{N}={ }_{2 N / \eta}^{\iota} \mathfrak{m}$. Since $\mathfrak{m}_{N} \subset \mathfrak{g}_{\underline{N}}$, we have $\mathfrak{m}_{N} \subset{ }_{2 N / \eta}^{\iota} \mathfrak{g}_{\underline{N}}$ hence $\mathfrak{m}_{N} \subset \tilde{\mathfrak{l}}_{N}$. Taking sum over all $N \in \mathbf{Z}$ such that $2 N / \eta \in \mathbf{Z}$, we get $\mathfrak{m} \subset \tilde{\mathfrak{l}}$. We can find $t_{0} \in \mathbf{k}^{*}$, $z \in \mathcal{Z}_{M}^{0}$, both of finite order, such that $\mathfrak{m}=\left\{y \in \mathfrak{g} ; \operatorname{Ad}\left(\iota\left(t_{0}\right)\right) \operatorname{Ad}(z) \theta(y)=y\right\}$. Note that $\tilde{\mathfrak{l}}_{*}=\dot{\eta} \tilde{\mathfrak{l}}_{*}(|\eta| / 2) \iota$

By $2.6(\mathrm{c})$, we can find $\zeta^{\prime} \in \mathbf{k}^{*}$ such that $\left.\tilde{\mathfrak{l}}=\left\{y \in \mathfrak{g} ; \operatorname{Ad}\left(\iota\left(\zeta^{\prime}\right)^{-1}\right) \theta(y)\right)=y\right\}$. Since $\mathfrak{m} \subset \tilde{\mathfrak{l}}$, we have:

(b) $\mathfrak{m}=\left\{y \in \tilde{\mathfrak{l}} ; \operatorname{Ad}\left(\iota\left(t_{0}\right)\right) \operatorname{Ad}(z) \theta(y)=y\right\}=\left\{y \in \tilde{\mathfrak{l}} ; \operatorname{Ad}\left(\iota\left(t_{0}\right)\right) \operatorname{Ad}(z) \operatorname{Ad}\left(\iota\left(\zeta^{\prime}\right)\right) y=y\right\}$.

(Note that 2.6(c) is applicable since $\tilde{\mathfrak{l}}_{*}=\dot{\eta} \tilde{\mathfrak{l}}_{*}^{(|\eta| / 2) \iota}$.)

Since $x \in \mathfrak{m}_{\eta} \subset{ }_{2}^{\iota} \mathfrak{g}$, we have $\operatorname{Ad}(\iota(t)) x=t^{2} x$ for any $t$. Taking $t=t_{0}^{-1}$ or $t=\zeta^{\prime}$ we see that $t_{0}^{-2} x=\operatorname{Ad}\left(\iota\left(t_{0}\right)\right)^{-1} x$ and $\zeta^{\prime 2} x=\operatorname{Ad}\left(\iota\left(\zeta^{\prime}\right)\right) x$. Since $x \in \mathfrak{m}$ and $x \in \tilde{\mathfrak{l}}$ we have $\operatorname{Ad}\left(\iota\left(t_{0}\right)\right)^{-1} x=\theta(x)$ and $\operatorname{Ad}\left(\iota\left(\zeta^{\prime}\right)\right) x=\theta(x)$. It follows that $t_{0}^{-2} x=\zeta^{\prime 2} x$ so that (since $x \neq 0$ ) we have $t_{0}^{-2}=\zeta^{\prime 2}$.

If $N \in \mathbf{Z}, 2 N / \eta \in \mathbf{Z}$ and $y \in \tilde{\mathfrak{l}}_{N}$, we have $\operatorname{Ad}\left(\iota\left(t_{0} \zeta^{\prime}\right)\right) y=\left(t_{0} \zeta^{\prime}\right)^{2 N / \eta} y=y$. Since $\tilde{\mathfrak{l}}=\oplus_{N} \tilde{\mathfrak{l}}_{N}$ we have $\operatorname{Ad}\left(\iota\left(t_{0} \zeta^{\prime}\right)\right) y=y$ for all $y \in \tilde{\mathfrak{l}}$. Hence (b) implies:

(c) $\mathfrak{m}=\{y \in \tilde{\mathfrak{l}} ; \operatorname{Ad}(z) y=y\}$.

It remains to show that (c) implies (a). Since $z$ is of finite order and $z \in \mathcal{Z}_{M}^{0}$, we can find $\lambda \in Y_{\mathcal{Z}_{M}^{0}}$ such that $z=\lambda\left(t_{1}\right)$ for some $t_{1} \in \mathbf{k}^{*}$. (See 3.2(a).)

Let $\mathfrak{m}^{\prime}=\left\{y \in \tilde{\mathfrak{l}} ; \operatorname{Ad}(\lambda(t)) y=y \quad \forall t \in \mathbf{k}^{*}\right\}$. Note that $\mathfrak{m}^{\prime}$ is a Levi subalgebra of a parabolic subalgebra $\mathfrak{q}$ of $\tilde{\mathfrak{l}}$. Since $\lambda\left(\mathbf{k}^{*}\right) \subset \mathcal{Z}_{M}^{0}$ we see that $\operatorname{Ad}(\lambda(t))$ acts as 1 on $\mathfrak{m}$ for any $t$ hence $\mathfrak{m} \subset \mathfrak{m}^{\prime}$. Now $\operatorname{Ad}\left(\lambda\left(t_{1}\right)\right)$ acts as 1 on $\mathfrak{m}^{\prime}$. Since $\mathfrak{m}=\{y \in$ $\left.\tilde{\mathfrak{l}} ; \operatorname{Ad}\left(\lambda\left(t_{1}\right)\right) y=y\right\}$ it follows that $\mathfrak{m}^{\prime}=\mathfrak{m}$. Thus (a) holds.

3.8. Primitive pairs. Let $\left(M, M_{0}, \mathfrak{m}, \mathfrak{m}_{*}, \tilde{C}\right) \in \mathfrak{T}_{\eta}$. Let $x \in \stackrel{\circ}{\mathfrak{m}}_{\eta}$. We can view $x$ as an element of $\mathfrak{g}_{\delta}^{\text {nil }}$. We set $M_{0}(x)=M_{0} \cap G(x), G_{0}(x)=G_{0} \cap G(x)$. We show:

(a) The inclusion $M_{0}(x) \rightarrow G_{0}(x)$ induces an isomorphism on the groups of components.

Let $\phi \in J^{M}, \tilde{\mathfrak{l}}, \tilde{\mathfrak{l}}_{*}, \tilde{L}$ be as in 3.7. Let $\tilde{L}_{0}=e^{\tilde{\mathfrak{l}}_{0}} \subset \tilde{L}$. We have $x \in \stackrel{\circ}{\mathfrak{l}}_{\eta}$ (see [L4, 4.2(a)]). Let $\tilde{L}_{0}(x)=\tilde{L}_{0} \cap G(x)$. To prove (a) it is enough to prove (i) and (ii) below. 
(i) The inclusion $M_{0}(x) \rightarrow \tilde{L}_{0}(x)$ induces an isomorphism on the groups of components.

(ii) The inclusion $\tilde{L}_{0}(x) \rightarrow G_{0}(x)$ induces an isomorphism on the groups of components.

Now (i) follows from [L4, 11.2] (we use 3.7(a)) and (ii) is a special case of 2.9(c). This proves (a).

Let $\mathcal{O}$ be the $G_{\underline{0}}$-orbit of $x$ in $\mathfrak{g}_{\delta}^{\text {nil }}$. Let $\mathcal{L}^{\prime}$ be the irreducible $M_{0}$-equivariant local system on $\stackrel{\circ}{\mathfrak{m}_{\eta}}$ such that $\left.\tilde{C}\right|_{\mathfrak{m}_{\eta}}=\mathcal{L}^{\prime}\left[\operatorname{dim} \mathfrak{m}_{\eta}\right]$. Let $\mathcal{L}$ be the irreducible $G_{\underline{0}^{-}}$ equivariant local system on $\mathcal{O}$ which corresponds to $\mathcal{L}^{\prime}$ under (a). We say that $(\mathcal{O}, \mathcal{L}) \in \mathcal{I}\left(\mathfrak{g}_{\delta}\right)$ is the primitive pair corresponding to $\left(M, M_{0}, \mathfrak{m}, \mathfrak{m}_{*}, \tilde{C}\right) \in \mathfrak{T}_{\eta} ;$ it is clearly independent of the choice of $x, \phi$ (we use [L4, 3.3]).

Let $\mathcal{L}^{\prime \prime}$ be the irreducible $\tilde{L}_{0}$-equivariant local system on $\stackrel{\circ}{\mathfrak{l}}_{\eta}$ which corresponds to $\mathcal{L}^{\prime}$ under (i). Let $\mathcal{L}^{\prime \prime \sharp} \in \mathcal{D}\left(\tilde{\mathfrak{l}}_{\eta}\right)$ be as in 0.11 . From $1.8(\mathrm{~b})$ we see that:

(b) $\operatorname{ind}_{\mathfrak{q}_{\eta}}^{\tilde{l}_{\eta}}(\tilde{C})$ is a nonzero direct sum of shifts of $\mathcal{L}^{\prime \prime \sharp}$.

Consider the map $\left(M, M_{0}, \mathfrak{m}, \mathfrak{m}_{*}, \tilde{C}\right) \mapsto(\mathcal{O}, \mathcal{L})$ (as above) from $\mathfrak{T}_{\eta}$ to $\mathcal{I}\left(\mathfrak{g}_{\delta}\right)$; the image of this map is denoted by $\mathcal{I}^{\text {prim }}\left(\mathfrak{g}_{\delta}\right)$. From 3.6(a) and (a) we see that:

(c) This induces a bijection $\omega: \underline{\mathfrak{T}}_{\eta} \stackrel{\sim}{\longrightarrow} \mathcal{I}^{\text {prim }}\left(\mathfrak{g}_{\delta}\right)$.

Using the definitions and $1.8(\mathrm{~b})$, we see that:

(d) For $\xi \in \underline{\mathfrak{T}}_{\eta}$ we have $\Psi(\omega(\xi))=\xi$, where $\Psi: \mathcal{I}\left(\mathfrak{g}_{\delta}\right) \rightarrow \underline{\mathfrak{T}}_{\eta}$ is as in 3.5 .

Combining (c) and (d), we have

(e) the restriction of $\Psi$ to $\mathcal{I}^{\text {prim }}\left(\mathfrak{g}_{\delta}\right)$ gives the inverse of $\omega$.

From (d) we get:

(f) The map $\Psi: \mathcal{I}\left(\mathfrak{g}_{\delta}\right) \rightarrow \underline{\mathfrak{T}}_{\eta}$ is surjective.

Another proof of (f) is given in 7.3.

3.9. Now let $\eta_{1} \in \mathbf{Z}-\{0\}$ be such that $\underline{\eta}_{1}=\delta$. We define a bijection $\mathfrak{T}_{\eta}^{\prime} \stackrel{\sim}{\rightarrow} \mathfrak{T}_{\eta_{1}}^{\prime}$ by $\left(M, M_{0}, \mathfrak{m}, \mathfrak{m}_{*}, \tilde{C}\right) \mapsto\left(M, M_{0}, \mathfrak{m}, \mathfrak{m}_{(*)}, \tilde{C}\right)$ where $\mathfrak{m}_{(*)}$ is the new Z-grading on $\mathfrak{m}_{*}$ whose $k$-component $\mathfrak{m}_{(k)}$ is equal to $\mathfrak{m}_{k \eta / \eta_{1}}$ for any $k \in \eta_{1} \mathbf{Z}$ and is equal to 0 for any $k \in \mathbf{Z}-\eta_{1} \mathbf{Z}$. (This is well defined since $\mathfrak{m}_{k^{\prime}}=0$ for any $k^{\prime} \in \mathbf{Z}-\eta \mathbf{Z}$; see 1.2(e).) Here we regard $\tilde{C}$ as a simple perverse sheaf on $\mathfrak{m}_{\eta}=\mathfrak{m}_{\left(\eta_{1}\right)}$. This restricts to a bijection $\mathfrak{T}_{\eta} \stackrel{\sim}{\rightarrow} \mathfrak{T}_{\eta_{1}}$, which induces a bijection $\underline{\mathfrak{T}}_{\eta} \stackrel{\sim}{\longrightarrow} \underline{\mathfrak{T}}_{\eta_{1}}$. This allows us to identify canonically all the sets $\mathfrak{T}_{\eta_{1}}$ (for various $\eta_{1} \in \mathbf{Z}-\{0\}$ such that $\underline{\eta}_{1}=\delta$ ) with a single set $\mathfrak{T}_{\delta}$ and also all the sets $\underline{\mathfrak{T}}_{\eta_{1}}$ (for various $\eta_{1} \in \mathbf{Z}-\{0\}$ such that $\underline{\eta}_{1}=\delta$ ) with a single set $\underline{\mathfrak{T}}_{\delta}$. Here $\mathfrak{T}_{\delta}, \underline{\mathfrak{T}}_{\delta}$ are defined purely in terms of $\delta$ (independently of the choice of $\eta$ ).

An examination of the construction of the map $\Psi=\Psi_{\eta}: \mathcal{I}\left(\mathfrak{g}_{\delta}\right) \rightarrow \underline{\mathfrak{T}}_{\eta}$ (see 3.5) shows that the bijection $\underline{\mathfrak{T}}_{\eta} \stackrel{\sim}{\rightarrow} \underline{\mathfrak{T}}_{\eta_{1}}$ intertwines $\Psi_{\eta}$ and $\Psi_{\eta_{1}}$. Therefore we have a well-defined map $\Psi: \mathcal{I}\left(\mathfrak{g}_{\delta}\right) \rightarrow \underline{\mathfrak{T}}_{\delta}$.

\section{Spiral induCtion}

In this section we introduce the key tool in studying the block decomposition for $\mathcal{D}_{G_{0}}\left(\mathfrak{g}_{\delta}^{\text {nil }}\right)$, namely the spiral induction. This is the analogue in the $\mathbf{Z} / \mathrm{m}$-graded setting of the parabolic induction in the ungraded or $\mathbf{Z}$-graded setting. 
4.1. Definition of spiral induction. In addition to $\eta \in \mathbf{Z}-\{0\}$ which has been fixed in 2.9 , in this section we fix $\epsilon \in\{1,-1\}$. We denote by $\mathfrak{P}^{\epsilon}$ the set of all data of the form:

$$
\left(\mathfrak{p}_{*}, L, P_{0}, \mathfrak{l}, \mathfrak{l}_{*}, \mathfrak{u}_{*}\right),
$$

where $\mathfrak{p}_{*}$ is an $\epsilon$-spiral and $L, P_{0}, \mathfrak{l}_{\mathfrak{l}} \mathfrak{l}_{*}, \mathfrak{u}_{*}$ are associated to $\mathfrak{p}_{*}$ as in 2.5. Let

$$
\left(\mathfrak{p}_{*}, L, P_{0}, \mathfrak{l}, \mathfrak{l}_{*}, \mathfrak{u}_{*}\right) \in \mathfrak{P}^{\epsilon} .
$$

Let $\pi: \mathfrak{p}_{\eta} \rightarrow \mathfrak{l}_{\eta}$ be the obvious projection. We have a diagram:

$$
\mathfrak{l}_{\eta} \stackrel{a}{\leftarrow} G_{\underline{0}} \times \mathfrak{p}_{\eta} \stackrel{b}{\rightarrow} E^{\prime} \stackrel{c}{\rightarrow} \mathfrak{g}_{\delta},
$$

where $E^{\prime}=\left\{\left(g P_{0}, z\right) \in G_{0} / P_{0} \times \mathfrak{g}_{\delta} ; \operatorname{Ad}\left(g^{-1}\right) z \in \mathfrak{p}_{\eta}\right\}, a(g, z)=\pi\left(\operatorname{Ad}\left(g^{-1}\right) z\right)$, $b(g, z)=\left(g P_{0}, z\right), c\left(g P_{0}, z\right)=z$. Here $a$ is smooth with connected fibers, $b$ is a principal $P_{0}$-bundle and $c$ is proper. Now $\mathcal{Q}\left(\mathfrak{l}_{\eta}\right)$ is defined as in 1.2 , with $H, \mathfrak{h}$ replaced by $L, \mathfrak{l}$. If $A \in \mathcal{Q}\left(\mathfrak{l}_{\eta}\right)$, then $a^{*} A$ is a $P_{0}$-equivariant semisimple complex on $G_{0} \times \mathfrak{p}_{\eta}$, hence there is a well-defined semisimple complex $A_{1}$ on $E^{\prime}$ such that $b^{*} A_{1}=a^{*} A$. We can form the complex

$$
{ }^{\epsilon} \operatorname{Ind}_{\mathfrak{p}_{\eta}}^{\mathfrak{g}_{\delta}}(A)=c_{!} A_{1} .
$$

Since $c$ is proper, this is a semisimple, $G_{\underline{0}}$-equivariant complex on $\mathfrak{g}_{\delta}$.

If $\tilde{\mathfrak{l}}_{*}$ is a splitting of $\mathfrak{p}_{*}$, we will sometimes consider ${ }^{\epsilon} \operatorname{Ind}_{\mathfrak{p}_{\eta}}^{\mathfrak{g}_{\delta}}(A)$ with $A \in \mathcal{Q}\left(\tilde{\mathfrak{l}}_{\eta}\right)$ by identifying $\tilde{\mathfrak{l}}_{\eta}$ with $\mathfrak{l}_{\eta}$ in an obvious way and $A$ with an object in $\mathcal{Q}\left(\mathfrak{l}_{\eta}\right)$.

For any $A \in \mathcal{Q}\left(\mathfrak{l}_{\eta}\right)$ we have

$$
D\left({ }^{\epsilon} \operatorname{Ind}_{\mathfrak{p}_{\eta}}^{\mathfrak{g}_{\delta}}(A)\right)={ }^{\epsilon} \operatorname{Ind}_{\mathfrak{p}_{\eta}}^{\mathfrak{g}_{\delta}}(D(A))[2 e],
$$

where $e$ is the dimension of a fiber of $a$ minus the dimension of a fiber of $b$, or equivalently

$$
\begin{aligned}
e & =\operatorname{dim} \mathfrak{g}_{\underline{0}}+\operatorname{dim} \mathfrak{p}_{\eta}-\operatorname{dim} \mathfrak{u}_{0}-\left(\operatorname{dim} \mathfrak{p}_{\eta}-\operatorname{dim} \mathfrak{u}_{\eta}\right)-\left(\operatorname{dim} \mathfrak{p}_{0}-\operatorname{dim} \mathfrak{u}_{0}\right) \\
& =\operatorname{dim} \mathfrak{u}_{0}+\operatorname{dim} \mathfrak{u}_{\eta} .
\end{aligned}
$$

Hence, if for $A \in \mathcal{Q}\left(\mathfrak{l}_{\eta}\right)$ we set

$$
\widetilde{\operatorname{Ind}}_{\mathfrak{p}_{\eta}}^{\mathfrak{g}_{\delta}}(A)={ }^{\epsilon} \operatorname{Ind}_{\mathfrak{p}_{\eta}}^{\mathfrak{g}_{\delta}}(A)\left[\operatorname{dim} \mathfrak{u}_{0}+\operatorname{dim} \mathfrak{u}_{\eta}\right],
$$

then

$$
D\left(\widetilde{\operatorname{Ind}_{\mathfrak{p}_{\eta}}} \mathfrak{g}_{\delta}(A)\right)=\widetilde{{ }^{\operatorname{Ind} d}}{\mathfrak{\mathfrak { p } _ { \eta }}}^{\mathfrak{g}_{\delta}}(D(A)) .
$$

4.2. Transitivity. We state a transitivity property of induction. In addition to the datum 4.1(a) we consider a parabolic subalgebra $\mathfrak{q}$ of $\mathfrak{l}$ such that $\mathfrak{q}=\oplus_{N \in \mathbf{Z}} \mathfrak{q}_{N}$ where $\mathfrak{q}_{N}=\mathfrak{q} \cap \mathfrak{l}_{N}$. For any $N \in \mathbf{Z}$ let $\hat{\mathfrak{p}}_{N}$ be the inverse image of $\mathfrak{q}_{N}$ under the obvious map $\mathfrak{p}_{N} \rightarrow \mathfrak{l}_{N}$. Then $\hat{\mathfrak{p}}_{*}$ is an $\epsilon$-spiral; see $2.8(\mathrm{a})$. Let

$$
\left(\hat{\mathfrak{p}}_{*}, \hat{L}, \hat{P}_{0}, \hat{\mathfrak{l}}, \hat{\mathfrak{l}}_{*}, \hat{\mathfrak{u}}_{*}\right) \in \mathfrak{P}^{\epsilon}
$$

be the datum analogous to 4.1 (a) defined by $\hat{\mathfrak{p}}_{*}$. Now $\mathcal{Q}\left(\hat{\mathfrak{l}}_{\eta}\right)$ is defined as in 1.2 , with $H, \mathfrak{h}$ replaced by $\hat{L}, \hat{\mathfrak{l}}$. If $A \in \mathcal{Q}\left(\hat{\mathfrak{l}}_{\eta}\right)$, then $\operatorname{ind}_{\mathfrak{q}_{\eta}}^{\mathfrak{l}_{\eta}}(A) \in \mathcal{Q}\left(\mathfrak{l}_{\eta}\right)$ is defined as in 1.3 and we have canonically

$$
{ }^{\epsilon} \operatorname{Ind}_{\hat{\mathfrak{p}}_{\eta}} \mathfrak{g}_{\eta}(A)={ }^{\epsilon} \operatorname{Ind}_{\mathfrak{p}_{\eta}}^{\mathfrak{g} \delta}\left(\operatorname{ind}_{\mathfrak{q}_{\eta}}^{\mathfrak{l}_{\eta}}(A)\right) .
$$

The proof is similar to that of [L2, 4.2]; it is omitted. 
4.3. In the setup of 4.1 , assume that $A \in \mathcal{Q}\left(\mathfrak{l}_{\eta}\right)$ is a cuspidal perverse sheaf (see 1.2). We have $A=\mathcal{L}^{\sharp}\left[\operatorname{dim} \mathfrak{l}_{\eta}\right]$ where $\mathcal{L}$ is an irreducible local system on $\stackrel{\mathfrak{l}}{\eta}_{\eta}$ and $\mathcal{L}^{\sharp} \in \mathcal{D}\left(\mathfrak{l}_{\eta}\right)$ is as in 0.11 . In this case we can give an alternative description of ${ }^{\epsilon} \operatorname{Ind}_{\mathfrak{p}_{\eta}}^{\mathfrak{g}_{\delta}}\left(\mathcal{L}^{\sharp}\right)$. Let $P_{0}, \pi: \mathfrak{p}_{\eta} \rightarrow \mathfrak{l}_{\eta}$ be as in 4.1. Let

$$
\left.\dot{\mathfrak{g}}_{\delta}=\left\{\left(g P_{0}, z\right) \in G_{\underline{0}} / P_{0} \times \mathfrak{g}_{\delta} ; \operatorname{Ad}\left(g^{-1}\right) z \in \pi^{-1}\left(\stackrel{\circ}{\mathfrak{l}}_{\eta}\right)\right)\right\}
$$

be an open smooth irreducible subvariety of $E^{\prime}$ in 4 .1. Let $\dot{\mathcal{L}}$ be the local system on $\dot{\mathfrak{g}}_{\delta}$ defined by $b^{\prime *} \dot{\mathcal{L}}=a^{* *} \mathcal{L}$, where

$$
\stackrel{\circ}{\mathfrak{l}}_{\eta} \stackrel{a^{\prime}}{\longleftarrow} G_{\underline{0}} \times\left(\pi^{-1}\left(\stackrel{\circ}{\mathfrak{l}}_{\eta}\right)\right) \stackrel{b^{\prime}}{\rightarrow} \dot{\mathfrak{g}}_{\delta},
$$

$a^{\prime}(g, z x)=\pi\left(\operatorname{Ad}\left(g^{-1}\right) z\right), b^{\prime}(g, z)=\left(g P_{0}, z\right)$. Let $\dot{\mathcal{L}}^{\sharp}$ be the intersection cohomology complex of $E^{\prime}$ with coefficients in $\dot{\mathcal{L}}$. From the definitions we have $a^{*} \mathcal{L}^{\sharp}=b^{*} \dot{\mathcal{L}}^{\sharp}$ $\left(a, b\right.$ as in 4.1). We define $c^{\prime}: \dot{\mathfrak{g}}_{\delta} \rightarrow \mathfrak{g}_{\delta}$ by $c^{\prime}(g, z)=z$. We show:

$$
{ }^{\epsilon} \operatorname{Ind}_{\mathfrak{p}_{\eta}}^{\mathfrak{g}_{\delta}}\left(\mathcal{L}^{\sharp}\right)=c_{!}^{\prime} \dot{\mathcal{L}} \text {. }
$$

Using the definitions we see that it is enough to show that the restriction of $\dot{\mathcal{L}}^{\sharp}$ to $E^{\prime}-\dot{\mathfrak{g}}_{\delta}$ is zero. This can be deduced from $1.2(\mathrm{c})$.

4.4. Let $\mathcal{Q}_{\eta}^{\epsilon}\left(\mathfrak{g}_{\delta}\right)$ be the subcategory of $\mathcal{D}\left(\mathfrak{g}_{\delta}\right)$ consisting of complexes which are direct sums of shifts of simple $G_{\underline{0}}$-equivariant perverse sheaves $B$ on $\mathfrak{g}_{\delta}$ with the following property: there exists a datum $\left(\mathfrak{p}_{*}, L, P_{0}, \mathfrak{l}, \mathfrak{l}_{*}, \mathfrak{u}_{*}\right)$ as in $4.1(\mathrm{a})$ and a simple cuspidal perverse sheaf $A$ in $\mathcal{Q}\left(\mathfrak{l}_{\eta}\right)$ such that some shift of $B$ is a direct summand of ${ }^{\epsilon} \operatorname{Ind}_{\mathfrak{p}_{\eta}}^{\mathfrak{g}_{\delta}}(A)$. We show:

(a) If $\left(\mathfrak{p}_{*}, L, P_{0}, \mathfrak{l}, \mathfrak{l}_{*}, \mathfrak{u}_{*}\right) \in \mathfrak{P}^{\epsilon}$ and $A^{\prime}$ is a simple (not necessarily cuspidal) perverse sheaf in $\mathcal{Q}\left(\mathfrak{l}_{\eta}\right)$, then ${ }^{\epsilon} \operatorname{Ind}_{\mathfrak{p}_{\eta}} \mathfrak{g}_{\delta}\left(A^{\prime}\right) \in \mathcal{Q}_{\eta}^{\epsilon}\left(\mathfrak{g}_{\delta}\right)$.

Using [L4, 7.5] we see that some shift of $A^{\prime}$ is a direct summand of $\operatorname{ind}_{\mathfrak{q}_{\eta}}^{\mathfrak{l}_{\eta}}(A)$ for some $\hat{\mathfrak{l}}, \mathfrak{q}$ as in 4.2 where $A$ is a simple cuspidal perverse sheaf in $\mathcal{Q}\left(\hat{\mathfrak{l}}_{\eta}\right)$. It follows that some shift of ${ }^{\epsilon} \operatorname{Ind}_{\mathfrak{p}_{\eta}}^{\mathfrak{g}_{\delta}}\left(A^{\prime}\right)$ is a direct summand of

$$
{ }^{\epsilon} \operatorname{Ind}_{\mathfrak{p}_{\eta}}^{\mathfrak{g}_{\delta}}\left(\operatorname{ind}_{\mathfrak{q}_{\eta}}^{\mathfrak{l}_{\eta}}(A)\right) .
$$

It is then enough to show that the complex (b) belongs to $\mathcal{Q}_{\eta}^{\epsilon}\left(\mathfrak{g}_{\delta}\right)$. This follows from the definitions using the transitivity property $4.2(\mathrm{a})$.

The functor

$$
{ }^{\epsilon} \operatorname{Ind}_{\mathfrak{p}_{\eta}}^{\mathfrak{g}_{\delta}}: \mathcal{Q}\left(\mathfrak{l}_{\eta}\right) \rightarrow \mathcal{Q}_{\eta}^{\epsilon}\left(\mathfrak{g}_{\delta}\right)
$$

(where $\left(\mathfrak{p}_{*}, L, P_{0}, \mathfrak{l}, \mathfrak{l}_{*}, \mathfrak{u}_{*}\right)$ is as in $4.1($ a) $)$ called spiral induction.

Let $\mathcal{K}_{\eta}^{\epsilon}\left(\mathfrak{g}_{\delta}\right)$ be the abelian group generated by symbols $(A)$, one for each isomorphism class of objects of $\mathcal{Q}_{\eta}^{\epsilon}\left(\mathfrak{g}_{\delta}\right)$, subject to the relations $(A)+\left(A^{\prime}\right)=\left(A \oplus A^{\prime}\right)$ (a Grothendieck group). Now $\mathcal{K}_{\eta}^{\epsilon}\left(\mathfrak{g}_{\delta}\right)$ is naturally an $\mathcal{A}$-module by $v^{n}(A)=(A[n])$ for any $n \in \mathbf{Z}$. We shall write $A$ instead of $(A)$ (in $\mathcal{Q}_{\eta}^{\epsilon}\left(\mathfrak{g}_{\delta}\right)$ ). Clearly, $\mathcal{K}_{\eta}^{\epsilon}\left(\mathfrak{g}_{\delta}\right)$ is a free $\mathcal{A}$-module with a finite distinguished basis given by the various simple perverse sheaves in $\mathcal{Q}_{\eta}^{\epsilon}\left(\mathfrak{g}_{\delta}\right)$. Now $A, B \mapsto(A: B)=\{A, D(B)\} \in \mathbf{N}((v))$ (see 0.12) defines a pairing

$$
(:): \mathcal{K}_{\eta}^{\epsilon}\left(\mathfrak{g}_{\delta}\right) \times \mathcal{K}_{\eta}^{\epsilon}\left(\mathfrak{g}_{\delta}\right) \rightarrow \mathbf{Z}((v)),
$$

which is $\mathcal{A}$-linear in the first argument, $\mathcal{A}$-antilinear in the second argument (for $f \mapsto \bar{f})$ and satisfies $\left(b_{1}: b_{2}\right)=\overline{\left(b_{2}: b_{1}\right)}$ for all $b_{1}, b_{2}$ in $\mathcal{K}_{\eta}^{\epsilon}\left(\mathfrak{g}_{\delta}\right)$. 
4.5. In addition to the datum 4.1(a) we consider another datum

$$
\left(\mathfrak{p}_{*}^{\prime}, L^{\prime}, P_{0}^{\prime}, \mathfrak{l}^{\prime}, \mathfrak{l}_{*}^{\prime}, \mathfrak{u}_{*}^{\prime}\right) \in \mathfrak{P}^{\epsilon}
$$

such that $\mathfrak{p}_{N} \subset \mathfrak{p}_{N}^{\prime}$ for all $N \in \mathbf{Z}$ and $\mathfrak{p}_{N}=\mathfrak{p}_{N}^{\prime}$ for $N \in\{\eta,-\eta\}$. We then have $\mathfrak{u}_{N}^{\prime} \subset \mathfrak{u}_{N}$ for all $N \in \mathbf{Z}$ and $\mathfrak{u}_{N}=\mathfrak{u}_{N}^{\prime}$ for $N \in\{\eta,-\eta\}$. We also have canonically $\mathfrak{l}_{N}=\mathfrak{l}_{N}^{\prime}$ for $N \in\{\eta,-\eta\}$ and $P_{0} \subset P_{0}^{\prime}$. Let $\mathcal{P}=P_{0}^{\prime} / P_{0}$. Write $\rho_{\mathcal{P} !} \overline{\mathbf{Q}}_{l}=\oplus_{j} \overline{\mathbf{Q}}_{l}\left[-2 a_{j}\right]$ where $a_{j}$ are integers $\geq 0$. (Here $\rho_{\mathcal{P}}$ ! is as in 0.12.) Let $A \in \mathcal{Q}\left(\mathfrak{l}_{\eta}\right)=\mathcal{Q}\left(\mathfrak{l}_{\eta}^{\prime}\right)$. We show:

(b) Let $I={ }^{\epsilon} \operatorname{Ind}_{\mathfrak{p}_{\eta}}^{\mathfrak{g}_{\delta}}(A), I^{\prime}={ }^{\epsilon} \operatorname{Ind}_{\mathfrak{p}_{\eta}^{\prime}}^{\mathfrak{g}_{\delta}}(A)$. We have $I \cong \oplus_{j} I^{\prime}\left[-2 a_{j}\right]$.

We consider the commutative diagram

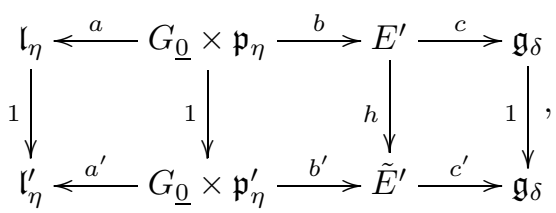

where the upper horizontal maps are as in 4.1(b), the lower horizontal are the analogous maps when 4.1 (a) is replaced by (a) and $h: E^{\prime} \rightarrow \tilde{E}^{\prime}$ is given by $\left(g P_{0}, z\right) \mapsto\left(g P_{0}^{\prime}, z\right)$. Note that $h$ is a $P_{0}^{\prime} / P_{0}$-bundle. We can find a complex $A_{1}$ (resp. $\left.A_{1}^{\prime}\right)$ on $E^{\prime}$ (resp. $\tilde{E}^{\prime}$ ) such that $I=c_{!} A_{1}, I^{\prime}=c_{!}^{\prime} A_{1}^{\prime}$. We have $A_{1}=h^{*} A_{1}^{\prime}$, hence

$$
I=c_{!} A_{1}=c_{!}^{\prime} h_{!} A_{1}=c_{!}^{\prime} h_{!} h^{*} A_{1}^{\prime}=c_{!}^{\prime}\left(A_{1}^{\prime} \otimes h_{!} h^{*} \overline{\mathbf{Q}}_{l}\right)=\oplus_{j} c_{!}^{\prime} A_{1}^{\prime}\left[-2 a_{j}\right]=\oplus_{j} I^{\prime}\left[-2 a_{j}\right] .
$$

This proves (b).

\section{StUdy OF A PAIR OF SPIRALS}

This section serves as preparation for the next one, which aims to calculate the Hom space between two spiral inductions.

5.1. In addition to $\eta \in \mathbf{Z}-\{0\}$ which has been fixed in 2.9 , in this section we fix $\epsilon^{\prime}, \epsilon^{\prime \prime}$ in $\{1,-1\}$. Let

$$
\left(\mathfrak{p}_{*}^{\prime}, L^{\prime}, P_{0}^{\prime}, \mathfrak{l}^{\prime}, \mathfrak{l}_{*}^{\prime}, \mathfrak{u}_{*}^{\prime}\right) \in \mathfrak{P}^{\epsilon^{\prime}}, \quad\left(\mathfrak{p}^{\prime \prime}, L^{\prime \prime}, P_{0}^{\prime \prime}, \mathfrak{l}^{\prime \prime}, \mathfrak{l}_{*}^{\prime \prime}, \mathfrak{u}_{*}^{\prime \prime}\right) \in \mathfrak{P}^{\epsilon^{\prime \prime}}
$$

We show:

(a) there exists a splitting $\tilde{\mathfrak{l}}_{*}^{\prime}$ of $\mathfrak{p}_{*}^{\prime}$ and a splitting $\tilde{\mathfrak{l}}_{*}^{\prime \prime}$ of $\mathfrak{p}_{*}^{\prime \prime}$ such that, if $\tilde{L}_{0}^{\prime}=$ $e^{\tilde{\boldsymbol{I}}_{0}^{\prime}} \subset G$ and $\tilde{L}_{0}^{\prime \prime}=e^{\tilde{\boldsymbol{I}}_{0}^{\prime \prime}} \subset G$, then some maximal torus $\mathcal{T}$ of $G_{0}$ is contained in both $\tilde{L}_{0}^{\prime}$ and $\tilde{L}_{0}^{\prime \prime}$.

Let $\tilde{\mathfrak{l}}_{*}^{\prime}$ be any splitting of $\mathfrak{p}_{*}^{\prime}$ and let $\tilde{\mathfrak{l}}_{*}^{\prime \prime}$ be any splitting of $\mathfrak{p}_{*}^{\prime \prime}$; let $\tilde{L}_{0}^{\prime}=e^{\tilde{\mathfrak{I}}_{0}^{\prime}} \subset G$, $\tilde{L}_{0}^{\prime \prime}=e^{{\tilde{I_{0}^{\prime \prime}}}_{0}} \subset G$. Recall that $P_{0}^{\prime}$ (resp. $P_{0}^{\prime \prime}$ ) is a parabolic subgroup of $G_{0}$ with Levi subgroup $\tilde{L}_{0}^{\prime}$ (resp. $\tilde{L}_{0}^{\prime \prime}$ ); hence there exists a maximal torus $\mathcal{T}_{0}$ of $G_{0}$ contained in both $P_{0}^{\prime}, P_{0}^{\prime \prime}$. Let ' $\tilde{L}_{0}^{\prime}$ (resp. ' $\left.\tilde{L}_{0}^{\prime \prime}\right)$ be the Levi subgroup of $P_{0}^{\prime}\left(\right.$ resp. $P_{0}^{\prime \prime}$ ) such that $\mathcal{T}_{0} \subset{ }^{\prime} \tilde{L}_{0}^{\prime}\left(\operatorname{resp} . \mathcal{T}_{0} \subset{ }^{\prime} \tilde{L}_{0}^{\prime \prime}\right)$. We can find $u^{\prime} \in U_{P_{0}^{\prime}}, u^{\prime \prime} \in U_{P_{0}^{\prime \prime}}$ such that $\operatorname{Ad}\left(u^{\prime}\right) \tilde{L}_{0}^{\prime}=$ ${ }^{\prime} \tilde{L}_{0}^{\prime}, \operatorname{Ad}\left(u^{\prime \prime}\right) \tilde{L}_{0}^{\prime \prime}={ }^{\prime} \tilde{L}_{0}^{\prime \prime}$. Now $\left\{\operatorname{Ad}\left(u^{\prime}\right) \tilde{\mathfrak{l}}_{N}^{\prime} ; N \in \mathbf{Z}\right\}$ is a splitting of $\left\{\operatorname{Ad}\left(u^{\prime}\right) \mathfrak{p}_{N}^{\prime} ; N \in\right.$ $\mathbf{Z}\}=\mathfrak{p}_{*}^{\prime}$ and $\left\{\operatorname{Ad}\left(u^{\prime \prime}\right) \tilde{\mathfrak{l}}_{N}^{\prime \prime} ; N \in \mathbf{Z}\right\}$ is a splitting of $\left\{\operatorname{Ad}\left(u^{\prime \prime}\right) \mathfrak{p}_{N}^{\prime \prime} ; N \in \mathbf{Z}\right\}=\mathfrak{p}_{*}^{\prime \prime}$. Note that $\operatorname{Ad}\left(u^{\prime}\right) \tilde{L}_{0}^{\prime}, \operatorname{Ad}\left(u^{\prime \prime}\right) \tilde{L}_{0}^{\prime \prime}$ contain a maximal torus of $G_{0} ;$ (a) is proved. 
5.2. Let $\left(\mathfrak{p}_{*}^{\prime}, L^{\prime}, P_{0}^{\prime}, \mathfrak{l}^{\prime}, \mathfrak{l}_{*}^{\prime}, \mathfrak{u}_{*}^{\prime}\right) \in \mathfrak{P}^{\epsilon^{\prime}},\left(\mathfrak{p}_{*}, L, P_{0}, \mathfrak{l}, \mathfrak{l}_{*}, \mathfrak{u}_{*}\right) \in \mathfrak{P}^{\epsilon^{\prime \prime}}$. Let $A \in \mathcal{Q}\left(\mathfrak{l}_{\eta}\right)$ be a simple cuspidal perverse sheaf. As in 4.3 , we have $A=\mathcal{L}^{\sharp}\left[\operatorname{dim} \mathfrak{l}_{\eta}\right]$ where $\mathcal{L}$ is an irreducible local system on $\stackrel{\circ}{\eta}_{\eta}$. Let

$$
B=\epsilon^{\prime} \operatorname{Ind}_{\mathfrak{p}_{\eta}}^{\mathfrak{g}_{\delta}}\left(\mathcal{L}^{\sharp}\right) .
$$

Let $\pi^{\prime}: \mathfrak{p}_{\eta}^{\prime} \rightarrow \mathfrak{l}_{\eta}^{\prime}$ be the obvious map with kernel $\mathfrak{u}_{\eta}^{\prime}$. We want to study the complex $K=\pi_{!}^{\prime}\left(\left.B\right|_{\mathfrak{p}_{\eta}^{\prime}}\right) \in \mathcal{D}\left(\mathfrak{l}_{\eta}^{\prime}\right)$. As in 4.3 , let

$$
\dot{\mathfrak{g}}_{\delta}=G_{\underline{0}} \stackrel{P_{0}}{\times} \pi^{-1}\left(\stackrel{\circ}{\mathfrak{l}}_{\eta}\right),
$$

where $\pi: \mathfrak{p}_{\eta} \rightarrow \mathfrak{l}_{\eta}$ is the obvious map; let $\dot{\mathcal{L}}$ be the local system on $\dot{\mathfrak{g}}_{\delta}$ defined in terms of $\mathcal{L}$ as in 4.3. As in 4.3, we define $c^{\prime}: \dot{\mathfrak{g}}_{\delta} \rightarrow \mathfrak{g}_{\delta}$ by $c^{\prime}(g, z)=z$. Let

$$
\dot{\mathfrak{p}}_{\eta}^{\prime}=\left\{\left(g P_{0}, z\right) \in G_{\underline{0}} / P_{0} \times \mathfrak{p}_{\eta}^{\prime} ; \operatorname{Ad}\left(g^{-1}\right) z \in \pi^{-1}\left(\stackrel{\mathfrak{l}}{\eta}_{\eta}\right)\right\} .
$$

Note that $\dot{\mathfrak{p}}_{\eta}^{\prime}$ is the closed subvariety $c^{\prime-1} \mathfrak{p}_{\eta}^{\prime}$ of $\dot{\mathfrak{g}}_{\delta}$. The restriction of $\dot{\mathcal{L}}$ from $\dot{\mathfrak{g}}_{\delta}$ to $\dot{\mathfrak{p}}_{\eta}^{\prime}$ is denoted again by $\dot{\mathcal{L}}$. Now $c^{\prime}$ restricts to a map $\dot{\mathfrak{p}}_{\eta}^{\prime} \rightarrow \mathfrak{p}_{\eta}^{\prime}$ whose composition with $\pi^{\prime}: \mathfrak{p}_{\eta}^{\prime} \rightarrow \mathfrak{l}_{\eta}^{\prime}$ is denoted by $\sigma: \dot{\mathfrak{p}}_{\eta}^{\prime} \rightarrow \mathfrak{l}_{\eta}^{\prime}$. We have $\sigma:\left(g P_{0}, z\right) \mapsto \pi^{\prime}(z)$. Using 4.3(a) and a proper base change, we see that $K=\sigma_{!}(\dot{\mathcal{L}})$.

We have a partition $\dot{\mathfrak{p}}_{\eta}^{\prime}=\cup_{\Omega} \dot{\mathfrak{p}}_{\eta, \Omega}^{\prime}$ into locally closed subvarieties indexed by the various $\left(P_{0}^{\prime}, P_{0}\right)$-double cosets $\Omega$ in $G_{\underline{0}}$, where

$$
\dot{\mathfrak{p}}_{\eta, \Omega}^{\prime}=\left\{\left(g P_{0}, z\right) \in \Omega / P_{0} \times \mathfrak{p}_{\eta}^{\prime} ; \operatorname{Ad}\left(g^{-1}\right) z \in \pi^{-1}\left(\stackrel{\circ}{l}_{\eta}\right)\right\} .
$$

Let $\sigma_{\Omega}: \dot{\mathfrak{p}}_{\eta, \Omega}^{\prime} \rightarrow \mathfrak{l}_{\eta}^{\prime}$ be the restriction of $\sigma$. For any $\Omega$ we set

$$
K_{\Omega}=\sigma_{\Omega !}\left(\left.\dot{\mathcal{L}}\right|_{\dot{\mathfrak{p}}_{\eta, \Omega}^{\prime}}\right) \in \mathcal{D}\left(\mathfrak{l}_{\eta}^{\prime}\right)
$$

We say that $\Omega$ is good if for some (or equivalently any) $g_{0} \in \Omega$, the following condition holds: setting $\mathfrak{p}_{N}^{\prime \prime}=\operatorname{Ad}\left(g_{0}\right) \mathfrak{p}_{N}, \mathfrak{u}_{N}^{\prime \prime}=\operatorname{Ad}\left(g_{0}\right) \mathfrak{u}_{N}$ (for $N \in \mathbf{Z}$ ), the obvious inclusion

$$
\left(\mathfrak{p}_{N}^{\prime} \cap \operatorname{Ad}\left(g_{0}\right) \mathfrak{p}_{N}\right) /\left(\mathfrak{p}_{N}^{\prime} \cap \operatorname{Ad}\left(g_{0}\right) \mathfrak{u}_{N}\right) \rightarrow \operatorname{Ad}\left(g_{0}\right) \mathfrak{p}_{N} / \operatorname{Ad}\left(g_{0}\right) \mathfrak{u}_{N}
$$

is an isomorphism for any $N \in \mathbf{Z}$ that is, $\operatorname{Ad}\left(g_{0}\right) \mathfrak{p}_{N}=\left(\mathfrak{p}_{N}^{\prime} \cap \operatorname{Ad}\left(g_{0}\right) \mathfrak{p}_{N}\right)+\operatorname{Ad}\left(g_{0}\right) \mathfrak{u}_{N}$. We say that $\Omega$ is bad if it is not good.

Until the end of 5.4 we fix an $\Omega$ as above and we choose $g_{0} \in \Omega$. Let $\mathfrak{p}_{N}^{\prime \prime}=$ $\operatorname{Ad}\left(g_{0}\right) \mathfrak{p}_{N}$; then $\mathfrak{p}_{*}^{\prime \prime}$ is an $\epsilon^{\prime \prime}$-spiral. It determines a datum $\left(\mathfrak{p}_{*}^{\prime \prime}, L^{\prime \prime}, P_{0}^{\prime \prime}, \mathfrak{l}^{\prime \prime}, \mathfrak{l}_{*}^{\prime \prime}, \mathfrak{u}_{*}^{\prime \prime}\right) \in$ $\mathfrak{P}^{\epsilon^{\prime \prime}}$.

By the change of variable $g=h g_{0}$ we may identify $\dot{\mathfrak{p}}_{\eta, \Omega}^{\prime}$ with

$$
\left.\left\{\left(h P_{0}^{\prime \prime}, z\right) \in P_{0}^{\prime} P_{0}^{\prime \prime} / P_{0}^{\prime \prime} \times \mathfrak{p}_{\eta}^{\prime} ; \operatorname{Ad}\left(h^{-1}\right) z \in \operatorname{Ad}\left(g_{0}\right) \pi^{-1}\left(\mathfrak{l}_{\eta}\right)\right)\right\}
$$

which is the same as

$$
\Xi=\left\{\left(h\left(P_{0}^{\prime} \cap P_{0}^{\prime \prime}\right), z\right) \in P_{0}^{\prime} /\left(P_{0}^{\prime} \cap P_{0}^{\prime \prime}\right) \times \mathfrak{p}_{\eta}^{\prime} ; \operatorname{Ad}\left(h^{-1}\right) z \in \pi^{\prime \prime-1}\left(\stackrel{\circ}{\mathfrak{l}}_{\eta}^{\prime \prime}\right)\right\}
$$

(in which $\pi^{\prime \prime}: \mathfrak{p}_{\eta}^{\prime \prime} \rightarrow \mathfrak{l}_{\eta}^{\prime \prime}$ is the obvious map, with kernel $\mathfrak{u}_{\eta}^{\prime \prime}$ ). In these coordinates, $\sigma_{\Omega}: \dot{\mathfrak{p}}_{\eta, \Omega}^{\prime} \rightarrow \mathfrak{l}_{\eta}^{\prime}$ becomes $\left(h\left(P_{0}^{\prime} \cap P_{0}^{\prime \prime}\right), z\right) \mapsto \pi^{\prime}(z)$.

We choose a splitting $\tilde{\mathfrak{l}}_{*}^{\prime}$ of $\mathfrak{p}_{*}^{\prime}$ and a splitting $\tilde{\mathfrak{l}}_{*}^{\prime \prime}$ of $\mathfrak{p}_{*}^{\prime \prime}$ as in $5.1\left(\right.$ a); let $\tilde{L}_{0}^{\prime}, \tilde{L}_{0}^{\prime \prime}, \mathcal{T}$ be as in 5.1(a).

Let $\mu^{\prime}, \mu^{\prime \prime}$ be elements of $Y_{G_{0}}, \mathbf{Q}$ such that $\mathfrak{p}_{*}^{\prime}=\epsilon^{\prime} \mathfrak{p}_{*}^{\mu^{\prime}}, \tilde{\mathfrak{l}}_{*}^{\prime}=\epsilon^{\prime} \tilde{\mathfrak{l}}_{*}^{\mu^{\prime}}, \mathfrak{p}_{*}^{\prime \prime}=\epsilon^{\prime \prime} \mathfrak{p}_{*}^{\mu^{\prime \prime}}$, $\tilde{\mathfrak{l}}_{*}^{\prime \prime}=\epsilon^{\prime \prime} \tilde{\mathfrak{l}}_{*}^{\mu^{\prime \prime}}$. Let $r^{\prime}, r^{\prime \prime}$ in $\mathbf{Z}_{>0}$ be such that $\lambda^{\prime}:=r^{\prime} \mu^{\prime} \in Y_{G_{\underline{0}}}, \lambda^{\prime \prime}:=r^{\prime \prime} \mu^{\prime \prime} \in Y_{G_{\underline{0}}}$. 
As in 2.6(d) we have $\lambda^{\prime}\left(\mathbf{k}^{*}\right) \subset \mathcal{Z}_{\tilde{L}_{0}^{\prime}}^{0}, \lambda^{\prime \prime}\left(\mathbf{k}^{*}\right) \subset \mathcal{Z}_{\tilde{L}_{0}^{\prime \prime}}^{0}$. Since $\mathcal{T}$ is a maximal torus of $\tilde{L}_{0}^{\prime}$, we must have $\mathcal{Z}_{\tilde{L}_{0}^{\prime}}^{0} \subset \mathcal{T}$ hence $\lambda^{\prime}\left(\mathbf{k}^{*}\right) \subset \mathcal{T}$. Similarly, since $\mathcal{T}$ is a maximal torus of $\tilde{L}_{0}^{\prime \prime}$, we have $\mathcal{Z}_{\tilde{L}_{0}^{\prime \prime}}^{0} \subset \mathcal{T}$ hence $\lambda^{\prime \prime}\left(\mathbf{k}^{*}\right) \subset \mathcal{T}$. Since both $\lambda^{\prime}\left(\mathbf{k}^{*}\right), \lambda^{\prime \prime}\left(\mathbf{k}^{*}\right)$ are contained in the torus $\mathcal{T}$, we must have $\lambda^{\prime}\left(t^{\prime}\right) \lambda^{\prime \prime}\left(t^{\prime \prime}\right)=\lambda^{\prime \prime}\left(t^{\prime \prime}\right) \lambda^{\prime}\left(t^{\prime}\right)$ for any $t^{\prime}, t^{\prime \prime}$ in $\mathbf{k}^{*}$. Hence, if for any $k^{\prime}, k^{\prime \prime}$ in $\mathbf{Z}$ and $i \in \mathbf{Z} / m$ we set

$$
k^{\prime}, k^{\prime \prime} \mathfrak{g}_{i}=\left\{x \in \mathfrak{g}_{i} ; \operatorname{Ad}\left(\lambda^{\prime}\left(t^{\prime}\right)\right) x=t^{\prime k^{\prime}} x, \operatorname{Ad}\left(\lambda^{\prime \prime}\left(t^{\prime \prime}\right)\right) x=t^{\prime \prime k^{\prime \prime}} x, \quad \forall t^{\prime}, t^{\prime \prime} \in \mathbf{k}^{*}\right\},
$$

then we have $\mathfrak{g}=\oplus_{k^{\prime}, k^{\prime \prime}, i}\left(k^{\prime}, k^{\prime \prime} \mathfrak{g}_{i}\right)$.

For any $N \in \mathbf{Z}$ we have a direct sum decomposition

$$
\mathfrak{p}_{N}^{\prime} \cap \mathfrak{p}_{N}^{\prime \prime}=\left(\tilde{\mathfrak{l}}_{N}^{\prime} \cap \tilde{\mathfrak{l}}_{N}^{\prime \prime}\right) \oplus\left(\mathfrak{u}_{N}^{\prime} \cap \tilde{\mathfrak{l}}_{N}^{\prime \prime}\right) \oplus\left(\tilde{\mathfrak{l}}_{N}^{\prime} \cap \mathfrak{u}_{N}^{\prime \prime}\right) \oplus\left(\mathfrak{u}_{N}^{\prime} \cap \mathfrak{u}_{N}^{\prime \prime}\right) .
$$

This follows immediately from the decompositions

$$
\begin{aligned}
& \mathfrak{p}_{N}^{\prime} \cap \mathfrak{p}_{N}^{\prime \prime}=\oplus_{k^{\prime}, k^{\prime \prime} ; k^{\prime} \geq N r^{\prime} \epsilon^{\prime}, k^{\prime \prime} \geq N r^{\prime \prime} \epsilon^{\prime \prime}}\left(k, k^{\prime} \mathfrak{g}_{\underline{N}}\right), \\
& \tilde{\mathfrak{l}}_{N}^{\prime} \cap \tilde{\mathfrak{l}}_{N}^{\prime \prime}=\oplus_{k^{\prime}, k^{\prime \prime} ; k^{\prime}=N r^{\prime} \epsilon^{\prime}, k^{\prime \prime}=N r^{\prime \prime} \epsilon^{\prime \prime}}\left(k, k^{\prime} \mathfrak{g}_{\underline{N}}\right), \\
& \mathfrak{u}_{N}^{\prime} \cap \tilde{\mathfrak{l}}_{N}^{\prime \prime}=\oplus_{k^{\prime}, k^{\prime \prime} ; k^{\prime}>N r^{\prime} \epsilon^{\prime}, k^{\prime \prime}=N r^{\prime \prime} \epsilon^{\prime \prime}}\left(k, k^{\prime} \mathfrak{g}_{\underline{N}}\right), \\
& \tilde{\mathfrak{l}}_{N}^{\prime} \cap \mathfrak{u}_{N}^{\prime \prime}=\oplus_{k^{\prime}, k^{\prime \prime} ; k^{\prime}=N r^{\prime} \epsilon^{\prime}, k^{\prime \prime}>N r^{\prime \prime} \epsilon^{\prime \prime}}\left(k, k^{\prime} \mathfrak{g}_{\underline{N}}\right), \\
& \mathfrak{u}_{N}^{\prime} \cap \mathfrak{u}_{N}^{\prime \prime}=\oplus_{k^{\prime}, k^{\prime \prime} ; k^{\prime}>N r^{\prime} \epsilon^{\prime}, k^{\prime \prime}>N r^{\prime \prime} \epsilon^{\prime \prime}}\left(k, k^{\prime} \mathfrak{g}_{\underline{N}}\right) .
\end{aligned}
$$

For $N \in \mathbf{Z}$ let $\mathfrak{q}_{N}^{\prime \prime}$ be the image of $\mathfrak{p}_{N}^{\prime} \cap \mathfrak{p}_{N}^{\prime \prime}$ under the obvious map $\mathfrak{p}_{N}^{\prime \prime} \rightarrow \mathfrak{l}_{N}^{\prime \prime}$; let $\mathfrak{q}^{\prime \prime}=\oplus_{N \in \mathbf{Z}}\left(\mathfrak{q}_{N}^{\prime \prime}\right)$, a Lie subalgebra of $\mathfrak{l}^{\prime \prime}$. We show:

(b) $\mathfrak{q}^{\prime \prime}$ is a parabolic subalgebra of $\mathfrak{l}^{\prime \prime}$ compatible with the $\mathbf{Z}$-grading of $\mathfrak{l}^{\prime \prime}$.

For $N \in \mathbf{Z}$ we set $\tilde{\mathfrak{q}}_{N}^{\prime \prime}=\tilde{\mathfrak{l}}_{N}^{\prime \prime} \cap \mathfrak{p}_{N}^{\prime}$. Let $\tilde{\mathfrak{q}}^{\prime \prime}=\oplus_{N \in \mathbf{Z}} \tilde{\mathfrak{q}}_{N}^{\prime \prime}$, a Lie subalgebra of $\tilde{\mathfrak{l}}^{\prime \prime}$. From (a) we see that the obvious isomorphism $\tilde{\mathfrak{l}}^{\prime \prime} \stackrel{\sim}{\longrightarrow} \mathfrak{l}^{\prime \prime}$ carries $\tilde{\mathfrak{q}}^{\prime \prime}$ to $\mathfrak{q}^{\prime \prime}$. Hence (b) follows from (c) below:

(c) $\tilde{\mathfrak{q}}^{\prime \prime}$ is a parabolic subalgebra of $\tilde{\mathfrak{l}}^{\prime \prime}$ compatible with the $\mathbf{Z}$-grading of $\tilde{\mathfrak{l}}^{\prime \prime}$.

We have

$$
\tilde{\mathfrak{q}}^{\prime \prime}=\oplus_{k^{\prime}, N \in \mathbf{Z} ; k^{\prime} \geq N r^{\prime} \epsilon^{\prime}}\left(k^{\prime}, N r^{\prime \prime} \epsilon^{\prime \prime} \mathfrak{g}_{\underline{N}}\right) .
$$

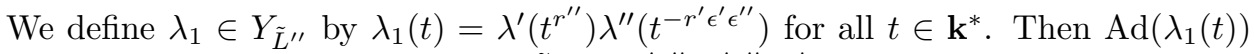
acts on the subspace $k^{\prime}, N r^{\prime \prime} \epsilon^{\prime \prime} \mathfrak{g}_{\underline{N}}$ of $\tilde{\mathfrak{l}}^{\prime \prime}$ as $t^{k^{\prime} r^{\prime \prime}-r^{\prime} r^{\prime \prime} N \epsilon^{\prime}}$; the last exponent of $t$ is $\geq 0$ if and only if $k^{\prime} \geq r^{\prime} N \epsilon^{\prime}$ which is just the condition that $k^{\prime}, N r^{\prime \prime} \epsilon^{\prime \prime} \mathfrak{g}_{\underline{N}}$ is one of the summands in the direct sum decomposition of $\tilde{\mathfrak{q}}^{\prime \prime}$. This proves (c).

For $N \in \mathbf{Z}$ let $\mathfrak{q}_{N}^{\prime}$ be the image of $\mathfrak{p}_{N}^{\prime} \cap \mathfrak{p}_{N}^{\prime \prime}$ under the obvious map $\mathfrak{p}_{N}^{\prime} \rightarrow \mathfrak{l}_{N}^{\prime}$; let $\mathfrak{q}^{\prime}=\oplus_{N \in \mathbf{Z}} \mathfrak{q}_{N}^{\prime}$, a Lie subalgebra of $\mathfrak{l}^{\prime}$.

For $N \in \mathbf{Z}$ we set $\tilde{\mathfrak{q}}_{N}^{\prime}=\tilde{\mathfrak{l}}_{N}^{\prime} \cap \mathfrak{p}_{N}^{\prime \prime}$. Let $\tilde{\mathfrak{q}}^{\prime}=\oplus_{N \in \mathbf{Z}} \tilde{\mathfrak{q}}_{N}^{\prime}$, a Lie subalgebra of $\tilde{\mathfrak{l}}^{\prime}$. The following result is proved in the same way as (b),(c).

(d) $\mathfrak{q}^{\prime}$ is a parabolic subalgebra of $\mathfrak{l}^{\prime}$ compatible with the $\mathbf{Z}$-grading of $\mathfrak{l}^{\prime} ; \tilde{\mathfrak{q}}^{\prime}$ is a parabolic subalgebra of $\tilde{\mathfrak{l}}^{\prime}$ compatible with the $\mathbf{Z}$-grading of $\tilde{\mathfrak{l}}^{\prime}$.

We set $\tilde{\mathfrak{q}}^{\prime \prime}=\oplus_{N} ! \tilde{\mathfrak{q}}_{N}^{\prime \prime}, ! \tilde{\mathfrak{q}}^{\prime}=\oplus_{N}\left({ }^{!} \tilde{\mathfrak{q}}_{N}^{\prime}\right)$, where

$$
! \tilde{\mathfrak{q}}_{N}^{\prime \prime}=\oplus_{k^{\prime} \in \mathbf{Z} ; k^{\prime}>N r^{\prime} \epsilon^{\prime}}\left(k^{\prime}, N r^{\prime \prime} \epsilon^{\prime \prime} \mathfrak{g}_{\underline{N}}\right), \quad ! \tilde{\mathfrak{q}}_{N}^{\prime}=\oplus_{k^{\prime \prime} \in \mathbf{Z} ; k^{\prime \prime}>N r^{\prime \prime} \epsilon^{\prime \prime}}\left(N r^{\prime} \epsilon^{\prime}, k^{\prime \prime} \mathfrak{g}_{\underline{N}}\right) .
$$

The proof of $(\mathrm{c})$ shows also that $\tilde{\mathfrak{q}}^{\prime \prime}$ is the nilradical of $\tilde{\mathfrak{q}}^{\prime \prime}$ and that

$$
\oplus_{N \in \mathbf{Z}}\left(N r^{\prime} \epsilon^{\prime}, N r^{\prime \prime} \epsilon^{\prime \prime} \mathfrak{g}_{\underline{N}}\right)
$$

is a Levi subalgebra of $\tilde{\mathfrak{q}}^{\prime \prime}$. Similarly, ! $\tilde{\mathfrak{q}}^{\prime}$ is the nilradical of $\tilde{\mathfrak{q}}^{\prime}$ and

$$
\oplus_{N \in \mathbf{Z}}\left(N r^{\prime} \epsilon^{\prime}, N r^{\prime \prime} \epsilon^{\prime \prime} \mathfrak{g}_{\underline{N}}\right)
$$


is a Levi subalgebra of $\tilde{\mathfrak{q}}^{\prime \prime}$. Thus,

(e) $\tilde{\mathfrak{q}}^{\prime}, \tilde{\mathfrak{q}}^{\prime \prime}$ have a common Levi subalgebra, namely $\oplus_{N \in \mathbf{Z}}\left(N r^{\prime} \epsilon^{\prime}, N r^{\prime \prime} \epsilon^{\prime \prime} \mathfrak{g}_{N}\right)$.

5.3. In this subsection we assume that $\Omega$ is bad. Then for some $N, \tilde{\mathfrak{l}}_{N}^{\prime \prime} \cap \mathfrak{p}_{N}^{\prime}$ is strictly contained in $\tilde{\mathfrak{l}}_{N}^{\prime \prime}$. Hence $\tilde{\mathfrak{q}}^{\prime \prime}$ is a proper parabolic subalgebra of $\tilde{\mathfrak{l}}^{\prime \prime}$ (see 5.2(c)). We will show that

$$
K_{\Omega}=\sigma_{\Omega !}\left(\left.\dot{\mathcal{L}}\right|_{\dot{\mathfrak{p}}_{\eta, \Omega}^{\prime}}\right)=0 \in \mathcal{D}\left(\mathfrak{l}_{\eta}^{\prime}\right)
$$

This is equivalent to the following statement:

(b) for any $y \in \tilde{\mathfrak{l}}_{\eta}^{\prime}$, the cohomology groups $H_{c}^{j}$ of the variety

$$
\left\{\left(h\left(P_{0}^{\prime} \cap P_{0}^{\prime \prime}\right), z\right) \in P_{0}^{\prime} /\left(P_{0}^{\prime} \cap P_{0}^{\prime \prime}\right) \times \mathfrak{p}_{\eta}^{\prime} ; z-y \in \mathfrak{u}_{\eta}^{\prime}, \operatorname{Ad}\left(h^{-1}\right) z \in \pi^{\prime \prime-1}\left(\stackrel{\circ}{\mathfrak{l}}_{\eta}^{\prime \prime}\right)\right\}
$$

with coefficients in the local system defined by $\dot{\mathcal{L}}$, are zero for all $j \in \mathbf{Z}$.

(We have identified $\tilde{\mathfrak{l}}_{\eta}^{\prime}, \mathfrak{l}_{\eta}^{\prime}$ via $\pi^{\prime}$.) Considering the fibers of the first projection of the last variety to $P_{0}^{\prime} /\left(P_{0}^{\prime} \cap P_{0}^{\prime \prime}\right)$, we see that it suffices to show that:

(c) for any $h \in P_{0}^{\prime}$ and any $y \in \tilde{\mathfrak{l}}_{\eta}^{\prime}$, the cohomology groups $H_{c}^{j}$ of the variety

$$
\left\{z \in \mathfrak{p}_{\eta}^{\prime} ; z-y \in \mathfrak{u}_{\eta}^{\prime}, \operatorname{Ad}\left(h^{-1}\right) z \in \stackrel{\circ}{\mathfrak{l}}_{\eta}^{\prime \prime}+\mathfrak{u}_{\eta}^{\prime \prime}\right\}
$$

with coefficients in the local system defined by $\dot{\mathcal{L}}$, are zero for all $j \in \mathbf{Z}$.

(We have used that $\pi^{\prime \prime-1}\left(\stackrel{\circ}{\mathfrak{l}}_{\eta}^{\prime \prime}\right)=\stackrel{\circ}{\tilde{\mathfrak{l}}_{\eta}^{\prime \prime}}+\mathfrak{u}_{\eta}^{\prime \prime}$.)

If $z$ is as in (c), then we have automatically $\operatorname{Ad}\left(h^{-1}\right) z \in \mathfrak{p}_{\eta}^{\prime}$; since $\tilde{\mathfrak{l}}_{\eta}^{\prime \prime}+\mathfrak{u}_{\eta}^{\prime \prime} \subset \mathfrak{p}_{\eta}^{\prime \prime}$, the condition that $\operatorname{Ad}\left(h^{-1}\right) z \in \stackrel{\circ}{\tilde{\mathfrak{l}}_{\eta}^{\prime \prime}}+\mathfrak{u}_{\eta}^{\prime \prime}$ implies $\operatorname{Ad}\left(h^{-1}\right) z \in \mathfrak{p}_{\eta}^{\prime} \cap \mathfrak{p}_{\eta}^{\prime \prime}$. By 5.2(a), we can then write uniquely $\operatorname{Ad}\left(h^{-1}\right) z=\gamma+\nu^{\prime}+\nu^{\prime \prime}+\mu$, where

$$
\gamma \in \tilde{\mathfrak{l}}_{\eta}^{\prime} \cap \tilde{\mathfrak{l}}_{\eta}^{\prime \prime}, \nu^{\prime} \in \mathfrak{u}_{\eta}^{\prime} \cap \tilde{\mathfrak{l}}_{\eta}^{\prime \prime}, \nu^{\prime \prime} \in \tilde{\mathfrak{l}}_{\eta}^{\prime} \cap \mathfrak{u}_{\eta}^{\prime \prime}, \mu \in \mathfrak{u}_{\eta}^{\prime} \cap \mathfrak{u}_{\eta}^{\prime \prime}
$$

The condition that $\operatorname{Ad}\left(h^{-1}\right) z \in \stackrel{\circ}{\mathfrak{l}}_{\eta}^{\prime \prime}+\mathfrak{u}_{\eta}^{\prime \prime}$ can be expressed as $\gamma+\nu^{\prime} \in \stackrel{\circ}{\mathfrak{I}}_{\eta}^{\prime \prime}$. The condition that $z-y \in \mathfrak{u}_{\eta}^{\prime}$ is equivalent to $\operatorname{Ad}\left(h^{-1}\right) z-\operatorname{Ad}\left(h^{-1}\right) y \in \mathfrak{u}_{\eta}^{\prime}$ or (if we define $y^{\prime} \in \tilde{\mathfrak{l}}_{\eta}^{\prime}$ by $\left.\operatorname{Ad}\left(h^{-1}\right) y-y^{\prime} \in \mathfrak{u}_{\eta}^{\prime}\right)$ to $\gamma+\nu^{\prime \prime}=y^{\prime}$. Note that $y^{\prime}, \gamma, \nu^{\prime \prime}$ are uniquely determined by $h, y$. Hence the variety in (c) can be identified with

$$
\left(\gamma+\left(\mathfrak{u}_{\eta}^{\prime} \cap \tilde{\mathfrak{l}}_{\eta}^{\prime \prime}\right)\right) \cap{\stackrel{\circ}{\tilde{\mathfrak{l}}_{\eta}^{\prime \prime}}}^{\prime \prime} \times\left(\mathfrak{u}_{\eta}^{\prime} \cap \mathfrak{u}_{\eta}^{\prime \prime}\right) .
$$

Under this identification, the local system $\dot{\mathcal{L}}$ is the pullback of $\mathcal{L}$ (viewed as a local system on $\stackrel{\circ}{\mathfrak{l}}_{\eta}^{\prime \prime}$ ) from the first factor. Now the desired vanishing of cohomology follows from the vanishing property [L4, 4.4(c)] of $\mathcal{L}$, since in our case $\tilde{\mathfrak{q}}^{\prime \prime}=\oplus_{N}\left(\tilde{\mathfrak{l}}_{N}^{\prime \prime} \cap \mathfrak{p}_{N}^{\prime}\right)$ is a proper parabolic subalgebra of $\tilde{\mathfrak{l}}^{\prime \prime}$ with nilradical $\oplus_{N}\left(\tilde{\mathfrak{l}}_{N}^{\prime \prime} \cap \mathfrak{u}_{N}^{\prime}\right)$.

5.4. In this subsection we assume that $\Omega$ is good. Then for any $N$ we have $\tilde{\mathfrak{l}}_{N}^{\prime \prime} \cap \mathfrak{p}_{N}^{\prime}=$ $\tilde{\mathfrak{l}}_{N}^{\prime \prime}$ that is, $\tilde{\mathfrak{l}}_{N}^{\prime \prime} \subset \mathfrak{p}_{N}^{\prime}$. We also have $\tilde{\mathfrak{q}}^{\prime \prime}=\tilde{\mathfrak{l}}^{\prime \prime}$. Thus $\tilde{\mathfrak{q}}^{\prime \prime}$ is reductive so it is equal to its Levi subalgebra $\oplus_{N \in \mathbf{Z}}\left(N r^{\prime} \epsilon^{\prime}, N r^{\prime \prime} \epsilon^{\prime \prime} \mathfrak{g}_{N}\right)$ (see 5.2(e)) which is then equal to $\tilde{\mathfrak{l}}^{\prime \prime}$ and is also a Levi subalgebra of $\tilde{\mathfrak{q}}^{\prime}$ (see $5.2(\mathrm{e})$ ). Thus,

(a) $\tilde{\mathfrak{l}}^{\prime \prime}$ is a Levi subalgebra of $\tilde{\mathfrak{q}}^{\prime}$.

Now $\operatorname{Ad}\left(g_{0}\right)$ defines an isomorphism $\mathfrak{l} \stackrel{\sim}{\rightarrow} \mathfrak{l}^{\prime \prime}$. Composing this with the inverse of the obvious isomorphism $\tilde{\mathfrak{l}}^{\prime \prime} \stackrel{\sim}{\longrightarrow} \mathfrak{l}^{\prime \prime}$ we obtain an isomorphism of Z-graded Lie 
algebras $\mathfrak{l} \stackrel{\sim}{\longrightarrow} \tilde{\mathfrak{l}}^{\prime \prime}$. Using this, we can transport $\mathcal{L}$ (a local system on $\stackrel{\mathfrak{l}}{\eta}_{\eta}$; see 5.1 ) to a local system $\mathcal{L}^{\prime \prime}$ on $\tilde{\mathfrak{l}}_{\eta}^{\prime \prime}$. Let $\mathcal{L}^{\prime \prime \sharp} \in \mathcal{D}\left(\tilde{\mathfrak{l}}_{\eta}^{\prime \prime}\right)$ be as in 0.11 . Then

$$
\operatorname{ind}_{\tilde{\mathfrak{q}}_{\eta}^{\prime}}^{\tilde{\mathfrak{l}}_{\eta}^{\prime}}\left(\mathcal{L}^{\prime \prime \sharp}\right) \in \mathcal{Q}\left(\tilde{\mathfrak{l}}_{\eta}^{\prime}\right)
$$

is defined as in 1.3 (we identify $\tilde{\mathfrak{l}}^{\prime \prime}$ with the reductive quotient of $\tilde{\mathfrak{q}}^{\prime}$; see (a)). We now state the following result.

(b) We have $K_{\Omega}=\operatorname{ind}_{\tilde{\mathfrak{q}}_{\eta}^{\prime}}^{\tilde{\tilde{l}}_{\eta}^{\prime}}\left(\mathcal{L}^{\prime \prime \sharp}\right)[-2 f]$, where

$$
f=\operatorname{dim} \mathfrak{u}_{0}^{\prime}-\operatorname{dim}\left(\mathfrak{u}_{0}^{\prime} \cap \mathfrak{p}_{0}^{\prime \prime}\right)+\operatorname{dim}\left(\mathfrak{u}_{\eta}^{\prime} \cap \mathfrak{u}_{\eta}^{\prime \prime}\right) .
$$

Let $\tilde{Q}_{0}^{\prime}=e^{\tilde{\mathfrak{q}}_{0}^{\prime}} \subset \tilde{L}_{0}^{\prime}$, a parabolic subgroup of $\tilde{L}_{0}^{\prime}$. Let

$$
\Xi^{\prime}=\left\{\left({ }^{\prime} h \tilde{Q}_{0}^{\prime},{ }^{\prime} z\right) \in \tilde{L}_{0}^{\prime} / \tilde{Q}_{0}^{\prime} \times \tilde{\mathfrak{l}}_{\eta}^{\prime} ; \operatorname{Ad}\left({ }^{\prime} h^{-1}\right)^{\prime} z \in \stackrel{\circ}{\tilde{\mathfrak{l}}_{\eta}^{\prime \prime}+!} \tilde{\mathfrak{q}}_{\eta}\right\}
$$

Define $c^{\prime \prime}: \Xi^{\prime} \rightarrow \tilde{\mathfrak{l}}_{\eta}^{\prime}$ by $c^{\prime \prime}\left({ }^{\prime} h Q_{0}^{\prime},{ }^{\prime} z\right)={ }^{\prime} z$. By the argument in [L4, 6.6] (for $\tilde{L}^{\prime}$ instead of $G$ ) we have

$$
\operatorname{ind}_{\tilde{\mathfrak{q}}_{\eta}^{\prime}}^{\tilde{\mathfrak{l}}_{\eta}^{\prime}}\left(\mathcal{L}^{\prime \prime \sharp}\right)=c_{!}^{\prime \prime} \dot{\mathcal{L}}^{\prime \prime},
$$

where $\dot{\mathcal{L}}^{\prime \prime}$ is a certain local system on $\Xi^{\prime}$ determined by $\mathcal{L}^{\prime \prime}$ and such that $\Delta^{*} \dot{\mathcal{L}}^{\prime \prime}=\dot{\mathcal{L}}$ where $\Delta: \Xi \rightarrow \Xi^{\prime}$ ( $\Xi$ as in 5.2 ) is the map induced by the canonical maps $P_{0}^{\prime} \rightarrow \tilde{L}_{0}^{\prime}$ (with kernel $\left.U_{P_{0}^{\prime}}\right)$ and $\mathfrak{p}_{\eta}^{\prime} \rightarrow \tilde{\mathfrak{l}}_{\eta}^{\prime}\left(\right.$ with kernel $\left.\mathfrak{u}_{\eta}^{\prime}\right) ; \dot{\mathcal{L}}$ is the local system on $\Xi$ considered in 5.2. We consider the following statement:

(d) $\Delta$ is an affine space bundle with fibers of dimension $f$.

Assuming that (d) holds, we have

$$
K_{\Omega}=c_{!}^{\prime \prime} \Delta_{!} \dot{\mathcal{L}}=c_{!}^{\prime \prime} \dot{\mathcal{L}}^{\prime \prime} \otimes \Delta_{!} \overline{\mathbf{Q}}_{l}=c_{!}^{\prime \prime} \dot{\mathcal{L}}^{\prime \prime}[-2 f]
$$

and we see that (b) follows from (c). It remains to prove (d).

Let ${ }^{\prime} h \in \tilde{L}_{0}^{\prime},{ }^{\prime} z \in \tilde{\mathfrak{l}}_{\eta}^{\prime}$ be such that $\left({ }^{\prime} h Q_{0}^{\prime},{ }^{\prime} z\right) \in \Xi^{\prime}$. Setting $h={ }^{\prime} h u, z={ }^{\prime} z+\tilde{z}$, we see that $\Delta^{-1}\left({ }^{\prime} h Q_{0}^{\prime},{ }^{\prime} z\right)$ can be identified with

$$
\left\{\left(u\left(U_{P_{0}^{\prime}} \cap P_{0}^{\prime \prime}\right), \tilde{z}\right) \in\left(U_{P_{0}^{\prime}} /\left(U_{P_{0}^{\prime}} \cap P_{0}^{\prime \prime}\right)\right) \times \mathfrak{u}_{\eta}^{\prime} ; \operatorname{Ad}\left(u^{-1}\right) \operatorname{Ad}\left({ }^{\prime} h^{-1}\right)\left({ }^{\prime} z+\tilde{z}\right) \in \stackrel{\circ}{\tilde{\mathfrak{l}}_{\eta}^{\prime \prime}}+\mathfrak{u}_{\eta}^{\prime \prime}\right\} .
$$

It suffices to show that

$$
\left\{(u, \tilde{z}) \in U_{P_{0}^{\prime}} \times \mathfrak{u}_{\eta}^{\prime} ; \operatorname{Ad}\left(u^{-1}\right) \operatorname{Ad}\left({ }^{\prime} h^{-1}\right)\left({ }^{\prime} z+\tilde{z}\right) \in \stackrel{\circ}{\tilde{\mathfrak{l}}_{\eta}^{\prime \prime}}+\mathfrak{u}_{\eta}^{\prime \prime}\right\}
$$

is isomorphic to $U_{P_{0}^{\prime}} \times\left(\mathfrak{u}_{\eta}^{\prime} \cap \mathfrak{u}_{\eta}^{\prime \prime}\right)$. If $(u, \tilde{z})$ are as in (e), we have automatically $\operatorname{Ad}\left(u^{-1}\right) \operatorname{Ad}\left({ }^{\prime} h^{-1}\right)\left({ }^{\prime} z+\tilde{z}\right) \in \mathfrak{p}_{\eta}^{\prime}\left(\right.$ since $^{\prime} z+\tilde{z} \in \mathfrak{p}_{\eta}^{\prime}$ and $\left.{ }^{\prime} h u \in P_{0}^{\prime}\right)$. Setting $\operatorname{Ad}\left({ }^{\prime} h^{-1}\right)^{\prime} z=$ $a \in \stackrel{\circ}{\mathfrak{l}}_{\eta}^{\prime \prime}+! \tilde{\mathfrak{q}}_{\eta}$ (where $a$ is fixed) and $\operatorname{Ad}\left(u^{-1}\right) \operatorname{Ad}\left({ }^{\prime} h^{-1}\right) \tilde{z}=\tilde{z}^{\prime} \in \mathfrak{u}_{\eta}^{\prime}$, we see that the variety (e) may be identified with the variety

$$
\left\{\left(u, \tilde{z}^{\prime}\right) \in U_{P_{0}^{\prime}} \times \mathfrak{u}_{\eta}^{\prime} ; \operatorname{Ad}\left(u^{-1}\right) a+\tilde{z}^{\prime} \in \stackrel{\circ}{\mathfrak{l}}_{\eta}^{\prime \prime}+\left(\mathfrak{p}_{\eta}^{\prime} \cap \mathfrak{u}_{\eta}^{\prime \prime}\right)\right\} .
$$

By 5.2(a) we can write uniquely

$$
\operatorname{Ad}\left(u^{-1}\right) a+\tilde{z}^{\prime}=\gamma+\nu+\mu,
$$


where $\gamma \in \stackrel{\circ}{\mathfrak{\mathfrak { l }}}_{\eta}^{\prime \prime}, \nu \in \tilde{\mathfrak{l}}_{\eta}^{\prime} \cap \mathfrak{u}_{\eta}^{\prime \prime}, \mu \in \mathfrak{u}_{\eta}^{\prime} \cap \mathfrak{u}_{\eta}^{\prime \prime}$. Setting $\hat{z}=\mu-\tilde{z}$ we see that (f) can be identified with the variety of all quintuples $\left(u, \hat{z}, \gamma, \nu, \nu^{\prime}\right)$ in

$$
U_{P_{0}^{\prime}} \times \mathfrak{u}_{\eta}^{\prime} \times \tilde{\mathfrak{l}}_{\eta}^{\prime \prime} \times\left(\tilde{\mathfrak{l}}_{\eta}^{\prime} \cap \mathfrak{u}_{\eta}^{\prime \prime}\right) \times\left(\mathfrak{u}_{\eta}^{\prime} \cap \mathfrak{u}_{\eta}^{\prime \prime}\right)
$$

such that

$$
\operatorname{Ad}\left(u^{-1}\right) a=\gamma+\nu+\hat{z}
$$

Since $a \in \tilde{\mathfrak{l}}_{\eta}^{\prime}$, we have $\operatorname{Ad}\left(u^{-1}\right) a-a \in \mathfrak{u}_{\eta}^{\prime}$ for $u \in U_{P_{0}^{\prime}}$. Hence in (g) we have $\gamma+\nu=a$ and $\hat{z}=\operatorname{Ad}\left(u^{-1}\right) a-a$. In particular, $\gamma, \nu$ are uniquely determined. Thus, our variety may be identified with $U_{P_{0}^{\prime}} \times\left(\mathfrak{u}_{N}^{\prime} \cap \mathfrak{u}_{N}^{\prime \prime}\right)$. This completes the proof of (d), hence that of (b).

5.5. From the results in 5.3 and 5.4 we can deduce, using the argument in L4, 8.9] (based on [L4, 1.4]), the following result.

Proposition 5.6. We have $K \in \mathcal{Q}\left(\mathfrak{l}_{\eta}^{\prime}\right)$; moreover, we have (noncanonically) $K \cong$ $\oplus_{\Omega} K_{\Omega}$, where $\Omega$ runs over good $\left(P_{0}^{\prime}, P_{0}\right)$-double cosets in $G_{\underline{0}}$.

\section{SPIRAL RESTRICTION}

We introduce the spiral restriction functor which is adjoint to the spiral induction. The main result in this section is Proposition 6.4, which calculates the inner product $\{$,$\} (in the sense of 0.12$ ) of two spiral inductions.

6.1. Definition of spiral restriction. In addition to $\eta \in \mathbf{Z}-\{0\}$ which has been fixed in 2.9 , in this section we fix $\epsilon^{\prime}, \epsilon^{\prime \prime}$ in $\{1,-1\}$. Let $\left(\mathfrak{p}_{*}^{\prime}, L^{\prime}, P_{0}^{\prime}, \mathfrak{l}^{\prime}, \mathfrak{l}_{*}^{\prime}, \mathfrak{u}_{*}^{\prime}\right) \in \mathfrak{P}^{\epsilon^{\prime}}$. Let $\pi^{\prime}: \mathfrak{p}_{\eta}^{\prime} \rightarrow \mathfrak{l}_{\eta}^{\prime}$ be the obvious map. For any $B \in \mathcal{D}\left(\mathfrak{g}_{\delta}\right)$ we set

$$
\epsilon^{\prime} \operatorname{Res}_{\mathfrak{p}_{\eta}^{\prime}}^{\mathfrak{g}_{\delta}}(B)=\pi_{!}^{\prime}\left(\left.B\right|_{\mathfrak{p}_{\eta}^{\prime}}\right) \in \mathcal{D}\left(\mathfrak{l}_{\eta}^{\prime}\right)
$$

We show:

(a) If $B \in \mathcal{Q}_{\eta}^{\epsilon^{\prime \prime}}\left(\mathfrak{g}_{\delta}\right)$, then $\epsilon^{\prime} \operatorname{Res}_{\mathfrak{p}_{\eta}^{\prime}}^{\mathfrak{g}_{\delta}}(B) \in \mathcal{Q}\left(\mathfrak{l}_{\eta}^{\prime}\right)$.

To prove this we can assume that $B$ is in addition a simple perverse sheaf. Then, using the definition of $\mathcal{Q}_{\eta}^{\epsilon^{\prime \prime}}\left(\mathfrak{g}_{\delta}\right)$, we see that it is enough to prove (a) in the case where $B=\epsilon^{\prime \prime} \operatorname{Ind}_{\mathfrak{p}_{\eta}}^{\mathfrak{g}_{\delta}}\left(\mathcal{L}^{\sharp}\right)$, with $\left(\mathfrak{p}_{*}, L, P_{0}, \mathfrak{l}, \mathfrak{l}_{*}, \mathfrak{u}_{*}\right) \in \mathfrak{P}^{\epsilon^{\prime \prime}}, \mathcal{L}^{\sharp}$ as in 5.2. In this case, (a) follows from 5.6.

We thus have a functor $\epsilon^{\prime} \operatorname{Res}_{\mathfrak{p}_{\eta}^{\prime}}^{\mathfrak{g}_{\delta}}: \mathcal{Q}_{\eta}^{\epsilon^{\prime \prime}}\left(\mathfrak{g}_{\delta}\right) \rightarrow \mathcal{Q}\left(\mathfrak{l}_{\eta}^{\prime}\right)$ called spiral restriction .

We have the following result.

Proposition 6.2 ((Adjunction)). Let $C \in \mathcal{Q}\left(\mathfrak{l}_{\eta}^{\prime}\right)$, and let $B \in \mathcal{Q}_{\eta}^{\epsilon^{\prime \prime}}\left(\mathfrak{g}_{\delta}\right)$. For any $j \in \mathbf{Z}$ we have

$$
d_{j}\left(\mathfrak{l}_{\eta}^{\prime} ; C,{ }^{\epsilon^{\prime}} \operatorname{Res}_{\mathfrak{p}_{\eta}^{\prime}}^{\mathfrak{g}_{\delta}}(B)\right)=d_{j^{\prime}}\left(\mathfrak{g}_{\delta} ; \epsilon^{\prime} \operatorname{Ind}_{\mathfrak{p}_{\eta}^{\prime}}^{\mathfrak{g} \delta}(C), B\right)
$$

where $j^{\prime}=j+2 \operatorname{dim} \mathfrak{u}_{0}^{\prime}$.

The proof is almost a copy of that of [L4, 9.2]. We omit it. 
For $B \in \mathcal{D}\left(\mathfrak{g}_{\delta}\right)$ we set

$$
\epsilon^{\prime} \widetilde{\operatorname{Res}}_{\mathfrak{p}_{\eta}^{\prime}}^{\mathfrak{g}_{\delta}}(B)={ }^{\epsilon^{\prime}} \operatorname{Res}_{\mathfrak{p}_{\eta}^{\prime}}^{\mathfrak{g}_{\delta}}(B)\left[\operatorname{dim} \mathfrak{u}_{\eta}^{\prime}-\operatorname{dim} \mathfrak{u}_{0}^{\prime}\right] .
$$

With this notation, the equality (a) can be reformulated without a shift from $j$ to $j^{\prime}$ as follows:

$$
d_{j}\left(\mathfrak{l}_{\eta}^{\prime} ; C, \epsilon^{\prime} \widetilde{\operatorname{Res}_{\mathfrak{p}_{\eta}^{\prime}}^{\mathfrak{g}_{\delta}}}(B)\right)=d_{j}\left(\mathfrak{g}_{\delta} ; \epsilon^{\epsilon^{\prime}} \widetilde{\operatorname{Ind}}_{\mathfrak{p}_{\eta}^{\prime}}^{\mathfrak{g}_{\delta}}(C), B\right)
$$

6.3. Let $\left(\mathfrak{p}_{*}^{\prime}, L^{\prime}, P_{0}^{\prime}, \mathfrak{l}^{\prime}, \mathfrak{l}_{*}^{\prime}, \mathfrak{u}_{*}^{\prime}\right) \in \mathfrak{P}^{\epsilon^{\prime}},\left(\mathfrak{p}_{*}, L, P_{0}, \mathfrak{l}, \mathfrak{l}_{*}, \mathfrak{u}_{*}\right) \in \mathfrak{P}^{\epsilon^{\prime \prime}}$. Let $A \in \mathcal{Q}\left(\mathfrak{l}_{\eta}\right)$, $A^{\prime} \in \mathcal{Q}\left(\mathfrak{l}_{\eta}^{\prime}\right)$ be cuspidal perverse sheaves. As in 4.3 we have $A=\mathcal{L}^{\sharp}\left[\operatorname{dim} \mathfrak{l}_{\eta}\right]$, $A^{\prime}=\mathcal{L}^{\prime \sharp}\left[\operatorname{dim} \mathfrak{l}_{\eta}^{\prime}\right]$ where $\mathcal{L}\left(\right.$ resp. $\left.\mathcal{L}^{\prime}\right)$ is a local system on $\stackrel{\circ}{\mathfrak{l}}_{\eta}\left(\right.$ resp. $\left.\stackrel{\circ}{\mathfrak{l}}_{\eta}^{\prime}\right)$.

We denote by $X$ the set of all $g \in G_{0}$ such that the $\epsilon^{\prime \prime}$-spiral $\left\{\operatorname{Ad}(g) \mathfrak{p}_{N} ; N \in \mathbf{Z}\right\}$ and the $\epsilon^{\prime}$-spiral $\mathfrak{p}_{*}^{\prime}$ have a common splitting. If $g \in X$ there is a unique isomorphism of Z-graded Lie algebras $\lambda_{g}: \mathfrak{l} \rightarrow \mathfrak{l}^{\prime}$ such that the compositions

$$
\begin{gathered}
\operatorname{Ad}(g) \mathfrak{p}_{N} \cap \mathfrak{p}_{N}^{\prime} \rightarrow \mathfrak{p}_{N}^{\prime} \rightarrow \mathfrak{l}_{N}^{\prime}, \\
\operatorname{Ad}(g) \mathfrak{p}_{N} \cap \mathfrak{p}_{N}^{\prime} \stackrel{\operatorname{Ad}\left(g^{-1}\right)}{\longrightarrow} \mathfrak{p}_{N} \rightarrow \mathfrak{l}_{N} \stackrel{\lambda_{g}}{\longrightarrow} \mathfrak{l}_{N}^{\prime}
\end{gathered}
$$

coincide for any $N$ (the unnamed maps are the obvious imbeddings or projections). Moreover, $\lambda_{g}$ is induced by an isomorphism $L \rightarrow L^{\prime}$. Let $X^{\prime}$ be the set of all $g \in X$ such that $\lambda_{g}: \mathfrak{l}_{\eta} \stackrel{\sim}{\rightarrow} \mathfrak{l}_{\eta}^{\prime}$ carries $\mathcal{L}$ to the dual of $\mathcal{L}^{\prime}$. For any $g \in X^{\prime}$ we set

$$
\tau(g)=-\operatorname{dim} \frac{\mathfrak{u}_{0}^{\prime}+\operatorname{Ad}(g) \mathfrak{u}_{0}}{\mathfrak{u}_{0}^{\prime} \cap \operatorname{Ad}(g) \mathfrak{u}_{0}}+\operatorname{dim} \frac{\mathfrak{u}_{\eta}^{\prime}+\operatorname{Ad}(g) \mathfrak{u}_{\eta}}{\mathfrak{u}_{\eta}^{\prime} \cap \operatorname{Ad}(g) \mathfrak{u}_{\eta}} .
$$

Note that both $X$ and $X^{\prime}$ are unions of $\left(P_{0}^{\prime}, P_{0}\right)$-double cosets in $G_{\underline{0}}$ and that $\tau(g)$ depends only on the double coset of $g$. We have the following result.

Proposition 6.4. Let

$$
\Pi=\sum_{j \in \mathbf{Z}} d_{j}\left(\mathfrak{g}_{\delta} ; \epsilon^{\prime} \widetilde{\operatorname{Ind}}_{\mathfrak{p}_{\eta}^{\prime}}^{\mathfrak{g}_{\delta}}\left(A^{\prime}\right), \epsilon^{\prime \prime} \widetilde{\operatorname{Ind}}_{\mathfrak{p}_{\eta}}^{\mathfrak{g}_{\delta}}(A)\right) v^{-j} \in \mathbf{N}((v)) .
$$

We have

$$
\Pi=\left(1-v^{2}\right)^{-r} \sum_{g_{0}} v^{\tau\left(g_{0}\right)},
$$

where $r$ is the dimension of the center of $\mathfrak{l}$ and the sum is taken over a set of representatives $g_{0}$ for the $\left(P_{0}^{\prime}, P_{0}\right)$-double cosets in $G_{\underline{0}}$ that are contained in $X^{\prime}$. In particular, if $\Pi \neq 0$, then $X^{\prime} \neq \emptyset$.

Using 6.2 , we have

$$
\Pi=\sum_{j \in \mathbf{Z}} d_{j}\left(\mathfrak{l}_{\eta}^{\prime} ; A^{\prime},{ }^{\epsilon^{\prime}} \widetilde{\operatorname{Res}}_{\mathfrak{p}_{\mathfrak{p}_{j}}^{\prime}}^{\mathfrak{g}_{\delta}}\left(\epsilon^{\prime \prime} \widetilde{\operatorname{Ind}}_{\mathfrak{p}_{\eta}}^{\mathfrak{g}_{\delta}}(A)\right)\right) v^{-j}=\sum_{j \in \mathbf{Z}} d_{j+s}\left(\mathfrak{l}_{\eta}^{\prime} ; A^{\prime}, K\right) v^{-j},
$$

where $s=\operatorname{dim} \mathfrak{u}_{0}+\operatorname{dim} \mathfrak{u}_{\eta}+\operatorname{dim} \mathfrak{u}_{\eta}^{\prime}-\operatorname{dim} \mathfrak{u}_{0}^{\prime}+\operatorname{dim} \mathfrak{l}_{\eta}$ and

$$
K=\epsilon^{\prime} \operatorname{Res}_{\mathfrak{p}_{\eta}^{\prime}}^{\mathfrak{g} \delta}\left(\epsilon^{\prime \prime} \operatorname{Ind}_{\mathfrak{p}_{\eta}}^{\mathfrak{g} \delta}\left(\mathcal{L}^{\sharp}\right)\right)
$$

is as in 5.2. Using the description of $K$ in 5.3(a), 5.4(b), 5.6, we see that

$$
\Pi=\sum_{j \in \mathbf{Z}} \sum_{g} Q_{j}(g) v^{-j+s-2 f(g)},
$$


where $g$ runs over a set of representatives for the $\left(P_{0}^{\prime}, P_{0}\right)$-double cosets in $G_{\underline{0}}$ which are good (see 5.2) and

$$
\begin{gathered}
Q_{j}(g)=d_{j}\left(\tilde{\mathfrak{l}}_{\eta}^{\prime} ; A^{\prime}, \operatorname{ind}_{\tilde{\mathfrak{q}}_{\eta}^{\prime}}^{\tilde{\mathfrak{l}}_{\eta}^{\prime}}\left(\mathcal{L}^{\prime \prime \sharp}\right)\right), \\
f(g)=\operatorname{dim}\left(\mathfrak{u}_{0}^{\prime} /\left(\mathfrak{u}_{0}^{\prime} \cap \operatorname{Ad}(g) \mathfrak{p}_{0}\right)+\operatorname{dim}\left(\mathfrak{u}_{\eta}^{\prime} \cap \operatorname{Ad}(g) \mathfrak{u}_{\eta}\right) ;\right.
\end{gathered}
$$

the following notation is used:

$\tilde{\mathfrak{l}}_{*}^{\prime}$ is a certain splitting of $\mathfrak{p}_{*}^{\prime}, \tilde{\mathfrak{l}}_{*}^{\prime \prime}$ is a certain splitting of $\left\{\operatorname{Ad}(g) \mathfrak{p}_{N} ; N \in \mathbf{Z}\right\}, \tilde{\mathfrak{q}}^{\prime}=$ $\oplus_{N \in \mathbf{Z}} \tilde{\mathfrak{q}}_{N}^{\prime}$ (where $\left.\tilde{\mathfrak{q}}_{N}^{\prime}=\tilde{\mathfrak{l}}_{N}^{\prime} \cap \operatorname{Ad}\left(g_{0}\right) \mathfrak{p}_{N}\right)$ is a parabolic subalgebra of $\tilde{\mathfrak{l}}^{\prime}=\oplus_{N} \tilde{\mathfrak{l}}_{N}^{\prime}$ whose with Levi subalgebra $\tilde{\mathfrak{l}}^{\prime \prime}=\oplus_{N} \tilde{\mathfrak{l}}_{N}^{\prime \prime} ; A^{\prime}$ is viewed as an object of $\mathcal{Q}\left(\tilde{\mathfrak{l}}_{\eta}^{\prime}\right)$ via the obvious isomorphism $\tilde{\mathfrak{l}}_{\eta}^{\prime} \rightarrow \mathfrak{l}_{\eta}^{\prime}$ and $\mathcal{L}^{\prime \prime \sharp} \in \mathcal{Q}\left(\tilde{\mathfrak{l}}_{\eta}^{\prime \prime}\right)$ corresponds to $\mathcal{L}^{\sharp}$ via the isomorphism $\mathfrak{l}_{\eta} \stackrel{\operatorname{Ad}(g)}{\longrightarrow} \operatorname{Ad}(g) \mathfrak{p}_{\eta} / \operatorname{Ad}(g) \mathfrak{u}_{\eta}=\tilde{\mathfrak{l}}_{\eta}^{\prime \prime}$.

By the implication (a) $\Longrightarrow(\mathrm{c})$ in $[\mathrm{L4}, 10.6]$, we have $Q_{j}(g)=0$ unless $\tilde{\mathfrak{q}}^{\prime}=\tilde{\mathfrak{l}}^{\prime}$. In this case, since $\tilde{\mathfrak{l}}^{\prime \prime}$ is a Levi subalgebra of $\tilde{\mathfrak{q}}^{\prime}$, we must have $\tilde{\mathfrak{l}}^{\prime}=\tilde{\mathfrak{l}}^{\prime \prime}$ so that $g \in X$. Conversely, if $g \in X$, then the $\left(P_{0}^{\prime}, P_{0}\right)$-double coset of $g$ is good. Indeed, let $\tilde{\mathfrak{l}}_{*}^{\prime}$ be a splitting of $\mathfrak{p}_{*}^{\prime}$ which is also a splitting for $\left\{\operatorname{Ad}(g) \mathfrak{p}_{N} ; N \in \mathbf{Z}\right\}$. We have

$$
\operatorname{Ad}(g) \mathfrak{p}_{N}=\tilde{\mathfrak{l}}_{N}^{\prime} \oplus \operatorname{Ad}(g) \mathfrak{u}_{N} \subset\left(\mathfrak{p}_{N}^{\prime} \cap \operatorname{Ad}(g) \mathfrak{p}_{N}\right)+\operatorname{Ad}(g) \mathfrak{u}_{N} \subset \operatorname{Ad}(g) \mathfrak{p}_{N}
$$

and our claim follows. Thus the sum in (a) can be taken over a set of representatives $g$ for the $\left(P_{0}^{\prime}, P_{0}\right)$-double cosets in $G_{0}$ that are contained in $X$ and for such $g$ we have $Q_{j}(g)=d_{j}\left(\tilde{\mathfrak{l}}_{\eta}^{\prime} ; A^{\prime}, \mathcal{L}^{\prime \prime \sharp}\right)$ where $\tilde{\mathfrak{l}}^{\prime}=\tilde{\mathfrak{l}}^{\prime \prime}, \mathcal{L}^{\prime \prime \sharp} \in \mathcal{Q}\left(\tilde{\mathfrak{l}}_{\eta}^{\prime \prime}\right)$ are as above. Using [L4, 15.1], we see that in the sum over $g$ in (a) we can take $g \in X^{\prime}$ and that the contribution of such $g$ to the sum is $\left(1-v^{2}\right)^{-r} v^{s-2 f(g)-d}$ where $d=\operatorname{dim} \mathfrak{l}_{\eta}$. It remains to show that for $g$ as above we have $s-2 f(g)-d=\tau(g)$. It is enough to show that:

(b) $\mathfrak{u}_{0}^{\prime} \cap \operatorname{Ad}(g) \mathfrak{p}_{0}=\mathfrak{u}_{0}^{\prime} \cap \operatorname{Ad}(g) \mathfrak{u}_{0}$,

(c) $\operatorname{dim}\left(\operatorname{Ad}(g) \mathfrak{u}_{0}\right)=\operatorname{dim} \mathfrak{u}_{0}^{\prime}$.

Now (b),(c) hold since $\operatorname{Ad}(g) \mathfrak{p}_{0}, \mathfrak{p}_{0}^{\prime}$ are parabolic subalgebras of $\mathfrak{g}_{0}$ with nilradicals $\operatorname{Ad}(g) \mathfrak{u}_{0}, \mathfrak{u}_{0}^{\prime}$ and with a common Levi subalgebra. This completes the proof of the proposition.

6.5. In the special case where

$$
\left(\mathfrak{p}_{*}^{\prime}, L^{\prime}, P_{0}^{\prime}, \mathfrak{l}^{\prime}, \mathfrak{l}_{*}^{\prime}, \mathfrak{u}_{*}^{\prime}\right)=\left(\mathfrak{p}_{*}, L, P_{0}, \mathfrak{l}, \mathfrak{l}_{*}, \mathfrak{u}_{*}\right)
$$

and $A^{\prime} \cong D(A)$, the sum $\sum_{g} v^{\tau(g)}$ in Proposition 6.4 is over a nonempty set of $g$ (we have $1 \in X^{\prime}$ ) hence the sum is nonzero and $\Pi$ in 6.4 is nonzero. In particular, we see that

$$
\epsilon^{\prime \prime} \widetilde{\operatorname{Ind}_{\mathfrak{p}_{\eta}}} \mathfrak{g}_{\delta}(A) \neq 0
$$

6.6. The map $\psi$ from simple perverse sheaves to $\underline{\mathfrak{T}}_{\eta}$. Let $B$ be a simple perverse sheaf in $\mathcal{Q}_{\eta}^{\epsilon^{\prime \prime}}\left(\mathfrak{g}_{\delta}\right)$. We associate to $B$ an element of $\underline{\mathfrak{I}}_{\eta}$ (see 3.5) as follows. We can find, $\left(\mathfrak{p}_{*}, L, P_{0}, \mathfrak{l}, \mathfrak{l}_{*}, \mathfrak{u}_{*}\right) \in \mathfrak{P}^{\epsilon^{\prime \prime}}$ and $A$ as in 6.3 such that

$$
\epsilon^{\prime \prime} \widetilde{\operatorname{Ind}}_{\mathfrak{p}_{\eta}}^{\mathfrak{g}_{\delta}}(A) \cong B[d] \oplus C,
$$

where $d \in \mathbf{Z}$ and $C \in \mathcal{Q}_{\eta}^{\epsilon^{\prime \prime}}\left(\mathfrak{g}_{\delta}\right)$. Let $\tilde{\mathfrak{l}}_{*}$ be a splitting of $\mathfrak{p}_{*}$. Let $\tilde{\mathfrak{l}}=\oplus_{N} \tilde{\mathfrak{l}}_{N}, \tilde{L}=$ $e^{\tilde{\mathfrak{l}}} \subset G, \tilde{L}_{0}=e^{\tilde{\mathfrak{l}}_{0}} \subset G$ and let $\tilde{C}$ be the simple perverse sheaf on $\tilde{\mathfrak{l}}_{\eta}$ corresponding to $A$ under the obvious isomorphism $\tilde{\mathfrak{l}}_{\eta} \stackrel{\sim}{\rightarrow} \mathfrak{l}_{\eta}$. Then $\left(\tilde{L}, \tilde{L}_{0}, \tilde{\mathfrak{l}}, \tilde{\mathfrak{l}}_{*}, \tilde{C}\right)$ is an object of 
$\mathfrak{T}_{\eta}$ and its $G_{\underline{0}^{-}}$orbit is independent of the choice of splitting, by $2.7(\mathrm{a})$. Now let $\left(\mathfrak{p}_{*}^{\prime}, L^{\prime}, P_{0}^{\prime}, \mathfrak{l}^{\prime}, \mathfrak{l}_{*}^{\prime}, \mathfrak{u}_{*}^{\prime}\right) \in \mathfrak{P}^{\epsilon^{\prime}}, A^{\prime}$ be as in 6.3 (with $\epsilon^{\prime}=\epsilon^{\prime \prime}$ ) and assume that

$$
\epsilon^{\prime} \widetilde{\operatorname{Ind}}_{\mathfrak{p}_{\eta}^{\prime}}^{\mathfrak{g}_{\delta}}\left(A^{\prime}\right) \cong B\left[d^{\prime}\right] \oplus C^{\prime}
$$

where $d^{\prime} \in \mathbf{Z}$ and $C^{\prime} \in \mathcal{Q}_{\eta}^{\epsilon^{\prime \prime}}\left(\mathfrak{g}_{\delta}\right)$. We choose a splitting $\tilde{\mathfrak{l}}_{*}^{\prime}$ of $\mathfrak{p}_{*}^{\prime}$ and we associate to it a system $\left(\tilde{L}^{\prime}, \tilde{L}_{0}^{\prime}, \tilde{\mathfrak{l}}^{\prime}, \tilde{\mathfrak{l}}_{*}^{\prime}, \tilde{C}^{\prime}\right)$ just as $\left(\tilde{L}, \tilde{L}_{0}, \tilde{\mathfrak{l}}, \tilde{\mathfrak{l}}_{*}, \tilde{C}\right)$ was defined in terms of $\tilde{\mathfrak{l}}$; here $\tilde{C}^{\prime}$ corresponds to $A^{\prime}$. Using $4.1(\mathrm{~d})$, we see that

$$
\epsilon^{\prime}{\widetilde{\operatorname{Ind}_{\mathfrak{p}_{\eta}^{\prime}}}}_{\mathfrak{g}_{\delta}}^{g}\left(D\left(A^{\prime}\right)\right) \cong D(B)\left[-d^{\prime}\right] \oplus D\left(C^{\prime}\right)
$$

Let $\Pi$ be as in 6.4 (with $A^{\prime}$ replaced by $D\left(A^{\prime}\right)$ and $\epsilon^{\prime}=\epsilon^{\prime \prime}$ ). From the definition of $\Pi$ in 6.4 we have also

$$
\Pi=\left\{B[d] \oplus C, D(B)\left[-d^{\prime}\right] \oplus D\left(C^{\prime}\right)\right\}=v^{d-d^{\prime}} \text { plus an element in } \mathbf{N}((v)) .
$$

(We use 0.12.) In particular we have $\Pi \neq 0$ hence $X^{\prime}$ in 6.4 is nonempty. It follows that $\left(\tilde{L}^{\prime}, \tilde{L}_{0}^{\prime}, \tilde{\mathfrak{l}}^{\prime}, \tilde{\mathfrak{l}}_{*}^{\prime}, \tilde{C}^{\prime}\right)$ and $\left(\tilde{L}, \tilde{L}_{0}, \tilde{\mathfrak{l}}, \tilde{\mathfrak{l}}_{*}, \tilde{C}\right)$ are in the same $G_{\underline{0}^{-}}$orbit. This proves that $B \mapsto\left(\tilde{L}, \tilde{L}_{0}, \tilde{\mathfrak{l}}, \tilde{\mathfrak{l}}_{*}, \tilde{C}\right)$ associates to $B$ a well-defined element $\psi(B) \in \underline{\mathfrak{T}}_{\eta}$.

6.7. For any $\xi \in \underline{\mathfrak{T}}_{\eta}$ let ${ }^{\xi} \mathcal{Q}_{\eta}^{\epsilon^{\prime}}\left(\mathfrak{g}_{\delta}\right)$ be the full subcategory of $\mathcal{Q}_{\eta}^{\epsilon^{\prime}}\left(\mathfrak{g}_{\delta}\right)$ whose objects are direct sums of shifts of simple perverse sheaves $B$ in $\mathcal{Q}_{\eta}^{\epsilon^{\prime}}\left(\mathfrak{g}_{\delta}\right)$ such that $\psi(B)=\xi$ (see 6.6); let ${ }^{\xi} \mathcal{K}_{\eta}^{\epsilon^{\prime}}\left(\mathfrak{g}_{\delta}\right)$ be the (free) $\mathcal{A}$-submodule of $\mathcal{K}_{\eta}^{\epsilon^{\prime}}\left(\mathfrak{g}_{\delta}\right)$ with basis given by the simple perverse sheaves $B$ in ${ }^{\xi} \mathcal{Q}_{\eta}^{\epsilon^{\prime}}\left(\mathfrak{g}_{\delta}\right)$. Clearly, we have

$$
\mathcal{K}_{\eta}^{\epsilon^{\prime}}\left(\mathfrak{g}_{\delta}\right)=\oplus_{\xi \in \underline{\mathfrak{T}}_{\eta}} \xi \mathcal{K}_{\eta}^{\epsilon^{\prime}}\left(\mathfrak{g}_{\delta}\right)
$$

\section{The CAtegories $\mathcal{Q}\left(\mathfrak{g}_{\delta}\right), \mathcal{Q}^{\prime}\left(\mathfrak{g}_{\delta}\right)$}

In this section we consider two categories of perverse sheaves $\mathcal{Q}\left(\mathfrak{g}_{\delta}\right), \mathcal{Q}^{\prime}\left(\mathfrak{g}_{\delta}\right)$ defined in terms of spiral induction; see 7.8. The simple objects in $\mathcal{Q}\left(\mathfrak{g}_{\delta}\right)$ are supported on $\mathfrak{g}_{\delta}^{\text {nil }}$, while those in $\mathcal{Q}^{\prime}\left(\mathfrak{g}_{\delta}\right)$ have Fourier-Deligne transforms supported on $\mathfrak{g}_{\delta}^{\text {nil }}$. We also complete the proof of the main theorem 0.6 .

7.1. Let $(\mathcal{O}, \mathcal{L}) \in \mathcal{I}\left(\mathfrak{g}_{\delta}\right)$. Let $A_{1}$ be the simple perverse sheaf on $\mathfrak{g}_{\delta}$ such that $\operatorname{supp}\left(A_{1}\right)$ is the closure $\overline{\mathcal{O}}$ of $\mathcal{O}$ in $\mathfrak{g}_{\delta}$ and $\left.A_{1}\right|_{\mathcal{O}}=\mathcal{L}[\operatorname{dim} \mathcal{O}]$.

Choose $x \in \mathcal{O}$ and $\phi \in J_{\delta}(x)$; define $\mathfrak{p}_{*}^{x}, \tilde{\mathfrak{l}}_{*}^{\phi}, \tilde{L}^{\phi}, P_{0}$ as in 2.9. Then $\mathcal{Q}\left(\tilde{\mathfrak{l}}_{\eta}^{\phi}\right)$ is defined in terms of $\tilde{\mathfrak{l}}_{*}^{\phi}, \tilde{L}^{\phi}$ and for any $A^{\prime} \in \mathcal{Q}\left(\tilde{\mathfrak{l}}_{\eta}^{\phi}\right)$ we can consider

$$
I\left(A^{\prime}\right):={ }^{\dot{\eta}} \operatorname{Ind}_{\mathfrak{p}_{\eta}^{x}}^{\mathfrak{g}_{\delta}}\left(A^{\prime}\right) \in \mathcal{Q}_{\eta}^{\dot{\eta}}\left(\mathfrak{g}_{\delta}\right)
$$

see 4.1. We show:

(a) If $A^{\prime} \in \mathcal{Q}\left(\tilde{\mathfrak{l}}_{\eta}^{\phi}\right)$, then the support of $I\left(A^{\prime}\right)$ is contained in $\overline{\mathcal{O}}$.

Let $y \in \mathfrak{g}_{\delta}$ be in the support of $I\left(A^{\prime}\right)$. We must show that $y \in \overline{\mathcal{O}}$. From the definition of $I\left(A^{\prime}\right)$, there exists $g \in G_{\underline{0}}$ and $z \in \mathfrak{p}_{\eta}^{x}$ such that $\operatorname{Ad}(g)(z)=y$. Since the support of $I\left(A^{\prime}\right)$ and $\overline{\mathcal{O}}$ are $G_{\underline{0}^{-}}$invariant we may replace $y$ by $\operatorname{Ad}\left(g^{-1}\right) y$ hence we may assume that $y \in \mathfrak{p}_{\eta}^{x}$. Using $2.9(\mathrm{e})$, we see that $\mathfrak{p}_{\eta}^{x}$ is equal to the closure of the $P_{0}$-orbit of $x$ in $\mathfrak{p}_{\eta}^{x}$, which is clearly contained in $\overline{\mathcal{O}}$. This proves (a). 


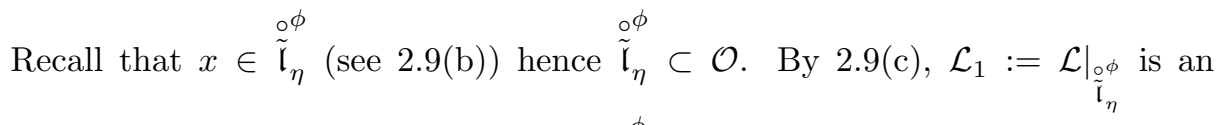
irreducible $\tilde{L}_{0}^{\phi}$-equivariant local system on $\stackrel{\stackrel{\circ}{\phi}}{\eta}_{\eta}$. Let $\mathcal{L}_{1}^{\sharp} \in \mathcal{D}\left(\tilde{\mathfrak{l}}_{\eta}^{\phi}\right)$ be as in 0.11 and let $A=\mathcal{L}_{1}^{\sharp}\left[\operatorname{dim} \tilde{\mathfrak{l}}_{\eta}^{\phi}\right]$. We show:

(b) $\left.I\left(\mathcal{L}_{1}^{\sharp}\right)\right|_{\mathcal{O}}$ is $\mathcal{L}$.

Let $E_{\mathcal{O}}^{\prime}$ be the inverse image of $\mathcal{O}$ under $c: E^{\prime} \rightarrow \mathfrak{g}_{\delta}$ (where $c, E^{\prime}$ are as in 4.1 with $\mathfrak{p}_{*}=\mathfrak{p}_{*}^{x}, \epsilon=\dot{\eta}$ ). From the definitions we see that it is enough to check that the map $c_{\mathcal{O}}: E_{\mathcal{O}}^{\prime} \rightarrow \mathcal{O}$ (restriction of $c$ ) is bijective on k-points. Since $G_{\underline{0}}$ acts naturally on both $E_{\mathcal{O}}^{\prime}$ and $\mathcal{O}$ compatibly with $c$ and the action on $\mathcal{O}$ is transitive, it suffices to check that $c^{-1}(x)$ is a single point, namely $\left(P_{0}, x\right)$. Let $\left(g P_{0}, x\right) \in c^{-1}(x)$. We have $g \in G_{\underline{0}}, \operatorname{Ad}\left(g^{-1}\right) x \in \mathfrak{p}_{\eta}^{x}$ hence $x \in \operatorname{Ad}(g) \mathfrak{p}_{\eta}^{x}$. From $2.9(\mathrm{~d})$ we deduce that $g \in P_{0}$ hence $\left(g P_{0}, x\right)=\left(P_{0}, x\right)$. This proves (b).

We show:

(c) $I\left(\mathcal{L}_{1}^{\sharp}\right)$ is isomorphic to $\oplus_{j=1}^{r} A_{j}\left[t_{j}\right]$, where $t_{1}=-\operatorname{dim} \mathcal{O}$ and for any $j \geq 2$, $A_{j}$ is a simple $G_{\underline{0}^{-}}$equivariant perverse sheaf on $\mathfrak{g}_{\delta}$ with support contained in $\overline{\mathcal{O}}-\mathcal{O}$ and $t_{j} \in \mathbf{Z}$.

This follows from the fact that $I\left(\mathcal{L}_{1}^{\sharp}\right)$ is a semisimple $G_{\underline{0}^{-}}$equivariant perverse sheaf on $\mathfrak{g}_{\delta}$ (the decomposition theorem), taking into account (a),(b).

By $1.5(\mathrm{a})$ we can find a parabolic subalgebra $\mathfrak{q}$ of $\tilde{\mathfrak{l}}^{\phi}$, a Levi subalgebra $\mathfrak{m}$ of $\mathfrak{q}$ (with $\mathfrak{q}, \mathfrak{m}$ compatible with the Z-grading of $\tilde{\mathfrak{l}}^{\phi}$ ) and a cuspidal $M_{0}:=e^{\mathfrak{m}_{0}}$ equivariant perverse sheaf $C$ on $\mathfrak{m}_{\eta}$ such that some shift of $A$ is a direct summand of $\operatorname{ind}_{\mathfrak{q}_{\eta}} \tilde{\tilde{i}}_{\eta}^{\phi}(C)$. From the definition we have

$$
\Psi(\mathcal{O}, \mathcal{L})=\left(M, M_{0}, \mathfrak{m}, \mathfrak{m}_{*}, C\right) \in \underline{\mathfrak{T}}_{\eta},
$$

where $M=e^{\mathfrak{m}} ;$ see 3.5 .

For any $N \in \mathbf{Z}$ let $\hat{\mathfrak{p}}_{N}$ be the inverse image of $\mathfrak{q}_{N}$ under the obvious map $\mathfrak{p}_{N} \rightarrow \mathfrak{l}_{N}$. Then by $2.8(\mathrm{a}), \hat{\mathfrak{p}}_{*}$ is an $\dot{\eta}$-spiral and $\mathfrak{m}_{*}$ is a splitting of it, so that, by $4.2(\mathrm{a})$, we have

$$
\dot{\eta} \operatorname{Ind}_{\hat{\mathfrak{p}}_{\eta}}^{\mathfrak{g}_{\delta}}(C)=\dot{\eta} \operatorname{Ind}_{\mathfrak{p}_{\eta}^{\phi}}^{\mathfrak{g}_{\delta}}\left(\operatorname{ind}_{\mathfrak{q}_{\eta}} \tilde{\mathfrak{i}}_{\eta}^{\phi}(C)\right) .
$$

It follows that some shift of $\dot{\eta} \operatorname{Ind}_{\mathfrak{p}_{\eta}^{\phi}}^{\mathfrak{g}_{\delta}}(A)$ is a direct summand of $\dot{\eta} \operatorname{Ind}_{\mathfrak{p}_{\eta}}^{\mathfrak{g} \delta}(C)$ hence, using (c), we see that some shift of $A_{1}$ is a direct summand of ${ }^{\dot{\eta}} \operatorname{Ind}_{\hat{\mathfrak{p}}_{\eta}}^{\mathfrak{g} \delta}(C)$. In particular we have $A_{1} \in \mathcal{Q}_{\eta}^{\dot{\eta}}\left(\mathfrak{g}_{\delta}\right)$ and $\psi\left(A_{1}\right)=\left(M, M_{0}, \mathfrak{m}, \mathfrak{m}_{*}, C\right) \in \underline{\mathfrak{I}}_{\eta}$; see 6.6 (with $\epsilon=\dot{\eta}$ ). Comparing with (d) we see that:

(e) $\psi\left(A_{1}\right)=\Psi(\mathcal{O}, \mathcal{L})$.

7.2. Characterization of $\mathcal{Q}_{\eta}^{\dot{\eta}}\left(\mathfrak{g}_{\delta}\right)$ as orbital sheaves. Let $A^{\prime}$ be a semisimple $G_{\underline{0}}$-equivariant complex on $\mathfrak{g}_{\delta}$. We show:

(a) We have $A^{\prime} \in \mathcal{Q}_{\eta}^{\dot{\eta}}\left(\mathfrak{g}_{\delta}\right)$ if and only if $\operatorname{supp}\left(A^{\prime}\right) \subset \mathfrak{g}_{\delta}^{\text {nil }}$.

We can assume that $A^{\prime}$ is a simple perverse sheaf. If $\operatorname{supp}\left(A^{\prime}\right) \subset \mathfrak{g}_{\delta}^{\text {nil }}$, then we have $A^{\prime} \in \mathcal{Q}_{\eta}^{\dot{\eta}}\left(\mathfrak{g}_{\delta}\right)$ by the arguments in 7.1. Conversely, assume that $A^{\prime} \in \mathcal{Q}_{\eta}^{\dot{\eta}}\left(\mathfrak{g}_{\delta}\right)$. We can find $\left(\mathfrak{p}_{*}, L, P_{0}, \mathfrak{l}, \mathfrak{l}_{*}, \mathfrak{u}_{*}\right) \in \mathfrak{P}^{\dot{\eta}}$ and $A \in \mathcal{Q}\left(\mathfrak{l}_{\eta}\right)$ such that some shift of $A^{\prime}$ is a direct summand of $B:=\dot{\eta} \operatorname{Ind}_{\mathfrak{p}_{\eta}}(A)$. To show that $\operatorname{supp}\left(A^{\prime}\right) \subset \mathfrak{g}_{\delta}^{\text {nil }}$ it is enough to show that $\operatorname{supp}(B) \subset \mathfrak{g}_{\delta}^{\text {nil }}$ or (with $c, A_{1}$ as in 4.1 with $\left.\epsilon=\dot{\eta}\right)$ that $\operatorname{supp}\left(c_{!} A_{1}\right) \subset \mathfrak{g}_{\delta}^{\text {nil }}$. This would follow if we can show that the image of $c$ is contained in $\mathfrak{g}_{\delta}^{\text {nil }}$. By the 
definition of $c$ it is enough to show that $\mathfrak{p}_{\eta} \subset \mathfrak{g}_{\delta}^{\text {nil }}$. This follows from 2.5(d) applied with $N=\eta$.

We now restate $7.1(\mathrm{e})$ as follows.

(b) Let $A^{\prime}$ be a simple perverse sheaf in $\mathcal{Q}_{\eta}^{\dot{\eta}}\left(\mathfrak{g}_{\delta}\right)$ and let $(\mathcal{O}, \mathcal{L}) \in \mathcal{I}\left(\mathfrak{g}_{\delta}\right)$ be such that $\operatorname{supp}\left(A^{\prime}\right)=\overline{\mathcal{O}}$ and $\left.A^{\prime}\right|_{\mathcal{O}}=\mathcal{L}[\operatorname{dim} \mathcal{O}]$. Then $\psi\left(A^{\prime}\right)=\Psi(\mathcal{O}, \mathcal{L})$. (Notation of 3.5 and 6.6 with $\epsilon=\dot{\eta}$.)

7.3. We now give another proof of the following statement (see also 3.8(f)):

(a) The map $\Psi: \mathcal{I}\left(\mathfrak{g}_{\delta}\right) \rightarrow \underline{\mathfrak{T}}_{\eta}$ in 3.5 is surjective.

Let $\left(M, M_{0}, \mathfrak{m}, \mathfrak{m}_{*}, C\right)$ be an element of $\mathfrak{T}_{\eta}$. We can find an $\dot{\eta}$-spiral $\mathfrak{p}_{*}$ such that $\mathfrak{m}_{*}$ is a splitting of $\mathfrak{p}_{*}$. By $6.5(\mathrm{a})$, we have ${ }^{\dot{\eta}} \widetilde{\operatorname{Ind}_{\mathfrak{p}_{\eta}}} \mathfrak{g}_{\delta}(C) \neq 0$, that is, there exists a simple perverse sheaf $A^{\prime}$ in $\mathcal{Q}_{\eta}^{\dot{\eta}}\left(\mathfrak{g}_{\delta}\right)$ such that some shift of $A^{\prime}$ is a direct summand of $\widetilde{\eta}^{\operatorname{Ind}_{\mathfrak{p}_{\eta}}} \mathfrak{g}_{\delta}(C)$. It follows that $\psi\left(A^{\prime}\right)=\left(M, M_{0}, \mathfrak{m}, \mathfrak{m}_{*}, C\right)$ hence, by $7.2(\mathrm{~b})$, we have $\Psi(\mathcal{O}, \mathcal{L})=\left(M, M_{0}, \mathfrak{m}, \mathfrak{m}_{*}, C\right)$ where $(\mathcal{O}, \mathcal{L})$ corresponds to $A^{\prime}$ as in $7.2(\mathrm{~b})$. This proves (a).

7.4. Until the end of 7.7 we assume that $p>0$. If $E, E^{\prime}$ are finite dimensional $\mathbf{k}$-vector space with a given perfect bilinear pairing $E \times E^{\prime} \rightarrow \mathbf{k}$, then we have the Fourier-Deligne transform functor $\Phi: \mathcal{D}(E) \rightarrow \mathcal{D}\left(E^{\prime}\right)$ defined in terms of a fixed nontrivial character $\mathbf{F}_{p} \rightarrow \overline{\mathbf{Q}}_{l}^{*}$ as in [L4, 1.9].

7.5. Fourier transform and spiral restriction. Let $B \in \mathcal{D}\left(\mathfrak{g}_{\delta}\right)$; we denote by $\Phi_{\mathfrak{g}}(B) \in \mathcal{D}\left(\mathfrak{g}_{-\delta}\right)$ the Fourier-Deligne transform of $B$ with respect to the perfect pairing $\mathfrak{g}_{\delta} \times \mathfrak{g}_{-\delta} \rightarrow \mathbf{k}$ defined by $\langle$,$\rangle .$

Let $\epsilon^{\prime} \in\{1,-1\}$. Let $\left(\mathfrak{p}_{*}^{\prime}, L^{\prime}, P_{0}^{\prime}, \mathfrak{l}^{\prime}, \mathfrak{l}_{*}^{\prime}, \mathfrak{u}_{*}^{\prime}\right) \in \mathfrak{P}^{\epsilon^{\prime}}$ and let

$$
R_{\eta}={ }^{\epsilon^{\prime}} \operatorname{Res}_{\mathfrak{p}_{\eta}^{\prime}}^{\mathfrak{g} \delta}(B) \in \mathcal{D}\left(\mathfrak{l}_{\eta}^{\prime}\right), \quad R_{-\eta}={ }^{\epsilon^{\prime}} \operatorname{Res}_{\mathfrak{p}_{-\eta}^{\prime}}^{\mathfrak{g}_{-\delta}}\left(\Phi_{\mathfrak{g}}(B)\right) \in \mathcal{D}\left(\mathfrak{l}_{-\eta}^{\prime}\right) .
$$

Then

(a) $R_{-\eta}$ is the Fourier-Deligne transform of $R_{\eta}$ with respect to the perfect pairing $\mathfrak{l}_{\eta} \times \mathfrak{l}_{-\eta} \rightarrow \mathbf{k}$ defined by $\langle$,$\rangle .$

The proof is almost the same as that of [L4, 10.2]. We omit it.

7.6. Fourier transform and spiral induction. Let $\epsilon^{\prime} \in\{1,-1\}$. Let

$$
\left(\mathfrak{p}_{*}^{\prime}, L^{\prime}, P_{0}^{\prime}, \mathfrak{l}^{\prime}, \mathfrak{l}_{*}^{\prime}, \mathfrak{u}_{*}^{\prime}\right) \in \mathfrak{P}^{\epsilon^{\prime}} .
$$

Let $A \in \mathcal{D}\left(\mathfrak{l}_{\eta}^{\prime}\right)$ be a semisimple complex; we denote by $\Phi_{\mathfrak{l}^{\prime}}(A) \in \mathcal{D}\left(\mathfrak{l}_{-\eta}^{\prime}\right)$ the Fourier-Deligne transform of $A$ with respect to the perfect pairing $\mathfrak{l}_{\eta}^{\prime} \times \mathfrak{l}_{-\eta}^{\prime} \rightarrow \mathbf{k}$ defined by $\langle$,$\rangle ; note that \Phi_{\mathfrak{l}^{\prime}}(A)$ is a semisimple complex. Let

$$
\begin{aligned}
& I_{\eta}=\epsilon^{\prime} \widetilde{\operatorname{Ind}_{\mathfrak{p}_{\eta}^{\prime}}} \mathfrak{g}^{\prime}(A) \in \mathcal{D}\left(\mathfrak{g}_{\delta}\right), \\
& I_{-\eta}=\epsilon^{\prime} \widetilde{\operatorname{Ind}_{\mathfrak{p}_{-\eta}^{\prime}}} \mathfrak{g}_{-\delta}\left(\Phi_{\mathfrak{l}^{\prime}}(A)\right) \in \mathcal{D}\left(\mathfrak{g}_{-\delta}\right) .
\end{aligned}
$$

Then:

(a) $I_{-\eta}$ is the Fourier-Deligne transform of $I_{\eta}$ with respect to the perfect pairing $\mathfrak{g}_{\delta} \times \mathfrak{g}_{-\delta} \rightarrow \mathbf{k}$ defined by $\langle$,$\rangle .$

The proof is almost the same as that of [L5, A2]. We omit it. 
7.7. Characterization of $\mathcal{Q}_{\eta}^{-\dot{\eta}}\left(\mathfrak{g}_{\delta}\right)$ as anti-orbital sheaves. Let $B \in \mathcal{D}\left(\mathfrak{g}_{\delta}\right)$ be a semisimple complex; let $B^{\prime}=\Phi_{\mathfrak{g}}(B) \in \mathcal{D}\left(\mathfrak{g}_{-\delta}\right)$ be its Fourier-Deligne transform, as in 7.5. Note that $B^{\prime}$ is again a semisimple complex. We show:

(a) We have $B \in \mathcal{Q}_{\eta}^{-\dot{\eta}}\left(\mathfrak{g}_{\delta}\right)$ if and only if $\operatorname{supp}\left(B^{\prime}\right) \subset \mathfrak{g}_{-\delta}^{\text {nil }}$.

We can assume that $B$ (and hence also $B^{\prime}$ ) is a simple perverse sheaf.

Assume first that $B \in \mathcal{Q}_{\eta}^{-\dot{\eta}}\left(\mathfrak{g}_{\delta}\right)$. We can find $\left(\mathfrak{p}_{*}^{\prime}, L^{\prime}, P_{0}^{\prime}, \mathfrak{l}^{\prime}, \mathfrak{l}_{*}^{\prime}, \mathfrak{u}_{*}^{\prime}\right) \in \mathfrak{P}^{-\dot{\eta}}$ and a cuspidal perverse sheaf $C$ in $\mathcal{Q}\left(\mathfrak{l}_{\eta}^{\prime}\right)$ such that some shift of $B$ is a direct summand of ${ }^{-\dot{\eta}} \widetilde{\operatorname{Ind}}_{\mathfrak{p}_{\eta}^{\prime}}^{\mathfrak{g}_{\delta}}(C)$. Using 7.6(a) we see that some shift of $B^{\prime}$ is a direct summand of ${ }^{-\dot{\eta}} \widetilde{\operatorname{Ind}_{\mathfrak{p}_{-\eta}^{\prime}}} \mathfrak{g}_{-\delta}\left(C^{\prime}\right)$ where $C^{\prime}=\Phi_{\mathfrak{l}^{\prime}}(C) \in \mathcal{D}\left(\mathfrak{l}_{-\eta}^{\prime}\right)$ (notation of 7.6). By [L4, 10.6], $C^{\prime}$ is a cuspidal perverse sheaf in $\mathcal{Q}\left(\mathfrak{l}_{-\eta}^{\prime}\right)$. It follows that $B^{\prime} \in \mathcal{Q}_{-\eta}^{-\dot{\eta}}\left(\mathfrak{g}_{-\delta}\right)$. Using 7.2(a) (with $\eta, \delta$ replaced by $-\eta,-\delta$ ) we deduce that $\operatorname{supp}\left(B^{\prime}\right) \subset \mathfrak{g}_{-\delta}^{\text {nil }}$.

Conversely, assume that $B$ is such that $\operatorname{supp}\left(B^{\prime}\right) \subset \mathfrak{g}_{-\delta}^{\text {nil }}$. Using 7.2(a), we see that $B^{\prime} \in \mathcal{Q}_{-\eta}^{-\dot{\eta}}\left(\mathfrak{g}_{-\delta}\right)$. We can find $\left(\mathfrak{p}_{*}^{\prime}, L^{\prime}, P_{0}^{\prime}, \mathfrak{l}^{\prime}, \mathfrak{l}_{*}^{\prime}, \mathfrak{u}_{*}^{\prime}\right) \in \mathfrak{P}^{-\dot{\eta}}$ and a cuspidal perverse sheaf $C_{1}^{\prime}$ in $\mathcal{Q}\left(\mathfrak{l}_{-\eta}^{\prime}\right)$ such that some shift of $B^{\prime}$ is a direct summand of $-\dot{\eta} \widetilde{\operatorname{Ind}_{\mathfrak{p}_{-\eta}^{\prime}}} \widetilde{\mathfrak{g}}_{-\delta}\left(C_{1}^{\prime}\right)$. We can find a cuspidal perverse sheaf $C_{1}$ in $\mathcal{Q}\left(\mathfrak{l}_{\eta}^{\prime}\right)$ such that $C_{1}^{\prime}=\Phi_{\mathfrak{l}^{\prime}}(C)$ (we use again [L4, 10.6]). Using 7.6(a), we see that some shift of $\Phi_{\mathfrak{g}}(B)$ is a direct summand of $\Phi_{\mathfrak{g}}\left({ }^{-} \dot{\eta} \widetilde{\operatorname{Ind}}_{\mathfrak{p}_{\eta}^{\prime}}^{\mathfrak{g}_{\delta}}\left(C_{1}\right)\right)$ hence some shift of $B$ is a direct summand of ${ }^{-} \dot{\operatorname{Ind}_{\mathfrak{p}_{\eta}^{\prime}}} \widetilde{\mathfrak{g}^{\prime}}\left(C_{1}\right)$ so that $B \in \mathcal{Q}_{\eta}^{-\dot{\eta}}\left(\mathfrak{g}_{\delta}\right)$. This completes the proof of (a).

7.8. The assumption on $p$ in 7.4 is no longer in force. From 7.2(a) we see that $\mathcal{Q}_{\eta}^{\dot{\eta}}\left(\mathfrak{g}_{\delta}\right)$ (hence also $\left.\mathcal{K}_{\eta}^{\dot{\eta}}\left(\mathfrak{g}_{\delta}\right)\right)$ is independent of $\eta$ as long as $\underline{\eta}=\delta$. We shall write $\mathcal{Q}\left(\mathfrak{g}_{\delta}\right), \mathcal{K}\left(\mathfrak{g}_{\delta}\right)$ instead of $\mathcal{Q}_{\eta}^{\dot{\eta}}\left(\mathfrak{g}_{\delta}\right), \mathcal{K}_{\eta}^{\dot{\eta}}\left(\mathfrak{g}_{\delta}\right)$. From $7.7(\mathrm{a})$ we see that $\mathcal{Q}_{\eta}^{-\dot{\eta}}\left(\mathfrak{g}_{\delta}\right)$ (hence also $\mathcal{K}_{\eta}^{-\dot{\eta}}\left(\mathfrak{g}_{\delta}\right)$ ) is independent of $\eta$ as long as $\underline{\eta}=\delta$ (at least when $p>0$, but then the same holds for $p=0$ by standard arguments). We shall write $\mathcal{Q}^{\prime}\left(\mathfrak{g}_{\delta}\right), \mathcal{K}^{\prime}\left(\mathfrak{g}_{\delta}\right)$ instead of $\mathcal{Q}_{\eta}^{-\dot{\eta}}\left(\mathfrak{g}_{\delta}\right), \mathcal{K}_{\eta}^{-\dot{\eta}}\left(\mathfrak{g}_{\delta}\right)$.

For $\xi \in \underline{\mathfrak{I}}_{\delta}$ we write ${ }^{\xi} \mathcal{Q}\left(\mathfrak{g}_{\delta}\right),{ }^{\xi} \mathcal{K}\left(\mathfrak{g}_{\delta}\right)$ instead of ${ }^{\xi} \mathcal{Q}_{\eta}^{\dot{\eta}}\left(\mathfrak{g}_{\delta}\right),{ }^{\xi} \mathcal{K}_{\eta}^{\dot{\eta}}\left(\mathfrak{g}_{\delta}\right)$ and we write ${ }^{\xi} \mathcal{Q}^{\prime}\left(\mathfrak{g}_{\delta}\right),{ }^{\xi} \mathcal{K}^{\prime}\left(\mathfrak{g}_{\delta}\right)$ instead of ${ }^{\xi} \mathcal{Q}_{\eta}^{-\dot{\eta}}\left(\mathfrak{g}_{\delta}\right),{ }^{\xi} \mathcal{K}_{\eta}^{-\dot{\eta}}\left(\mathfrak{g}_{\delta}\right)$. The discussion in 3.9 shows that ${ }^{\xi} \mathcal{Q}\left(\mathfrak{g}_{\delta}\right),{ }^{\xi} \mathcal{K}\left(\mathfrak{g}_{\delta}\right)$ and ${ }^{\xi} \mathcal{Q}^{\prime}\left(\mathfrak{g}_{\delta}\right),{ }^{\xi} \mathcal{K}^{\prime}\left(\mathfrak{g}_{\delta}\right)$ are independent of $\eta$ as long as $\underline{\eta}=\delta$.

7.9. Proof of Theorem 0.6. Let $\xi \in \underline{\mathfrak{I}}_{\eta}$. Let $K \in \mathcal{D}_{G_{\underline{0}}}\left(\mathfrak{g}_{\delta}^{\text {nil }}\right)$. We say that $K \in$ $\mathcal{D}_{G_{\underline{0}}}\left(\mathfrak{g}_{\delta}^{\text {nil }}\right)_{\xi}$ if any simple perverse sheaf $B$ which appears in a perverse cohomology sheaf of $K$ satisfies $\psi(B)=\xi$; note that $B$ belongs to $\mathcal{Q}_{\eta}^{\dot{\eta}}\left(\mathfrak{g}_{\delta}\right)$, see $7.2(\mathrm{a})$; hence $\psi(B)$ is defined as in 6.6 .

Now let $\xi, \xi^{\prime}$ in $\underline{\mathfrak{T}}_{\eta}$ be such that $\xi \neq \xi^{\prime}$. Let $K \in \mathcal{D}_{G_{\underline{0}}}\left(\mathfrak{g}_{\delta}^{\text {nil }}\right)_{\xi}, K^{\prime} \in \mathcal{D}_{G_{\underline{0}}}\left(\mathfrak{g}_{\delta}^{\text {nil }}\right)_{\xi^{\prime}}$. We show:

(a) $\operatorname{Hom}_{\mathcal{D}_{G_{\underline{0}}}\left(\mathfrak{g}_{\delta}^{\text {nil }}\right)}\left(K, K^{\prime}\right)=0$.

We can assume that $K=B[n], K^{\prime}=B^{\prime}\left[n^{\prime}\right]$ where $B, B^{\prime}$ are simple perverse sheaves in $\mathcal{Q}_{\eta}^{\dot{\eta}}\left(\mathfrak{g}_{\delta}\right)$ such that $\psi(B)=\xi, \psi\left(B^{\prime}\right)=\xi^{\prime}$ and $n, n^{\prime}$ are integers. We see that it is enough to prove (a) in the case where $K=\dot{\eta_{\operatorname{Ind}}} \widetilde{\mathfrak{g}_{\delta}}\left(A^{\prime}\right)[n], K^{\prime}=\dot{\eta_{\eta}} \widetilde{\operatorname{Ind}_{\mathfrak{p}_{\eta}}} \mathfrak{g}_{\delta}(A)\left[n^{\prime}\right]$ with $n, n^{\prime} \in \mathbf{Z}, \mathfrak{p}_{*}, \mathfrak{p}_{*}^{\prime}, A, A^{\prime}$ as in 6.4 , and $\epsilon^{\prime}=\epsilon^{\prime \prime}=\dot{\eta}$, since some shifts of $B$ and $B^{\prime}$ appear as direct summands of such $K$ and $K^{\prime}$. By $0.12($ a), we have an isomorphism

$$
\operatorname{Hom}_{\mathcal{D}_{G_{\underline{0}}}\left(\mathfrak{g}_{\delta}^{n i l}\right)}\left(K, K^{\prime}\right)=\mathbf{D}_{0}\left(\mathfrak{g}_{\delta}^{n i l}, G_{\underline{0}} ; K, D\left(K^{\prime}\right)\right)^{*} .
$$

Hence

(b) $\operatorname{dim} \operatorname{Hom}_{\mathcal{D}_{G_{0}}\left(\mathfrak{g}_{\delta}^{n i l}\right)}\left(K, K^{\prime}\right)=d_{n-n^{\prime}}\left(\mathfrak{g}_{\delta}^{n i l} ; \widetilde{\eta}_{\operatorname{Ind}_{\mathfrak{p}_{\eta}^{\prime}}^{\mathfrak{g}_{\delta}}}^{\mathfrak{g}^{\prime}}\left(A^{\prime}\right), \widetilde{\eta}_{\operatorname{Ind}_{\mathfrak{p}_{\eta}}}^{\mathfrak{g}_{\delta}}(D(A))\right)$. 
Here we use $4.1(\mathrm{~d})$. Since $\xi \neq \xi^{\prime}$, the set $X^{\prime}$ defined in 6.4 for the pair $\left(D(A), A^{\prime}\right)$ is empty. Therefore the right side of (b) is zero by 6.4. Then (a) follows from (b). We see that Theorem 0.6 holds.

\section{Monomial AND QUASI-MONOMIAL OBJECTS}

The results in this section are parallel to those in 1.8-1.9. They serve as preparation for the next section.

8.1. Let $\epsilon=\dot{\eta}$. We denote by $\mathfrak{R}^{\epsilon}$ the set of all data of the form

$$
\left(\mathfrak{p}_{*}, L, P_{0}, \mathfrak{l}, \mathfrak{l}_{*}, \mathfrak{u}_{*}, A\right),
$$

where $\left(\mathfrak{p}_{*}, L, P_{0}, \mathfrak{l}, \mathfrak{l}_{*}, \mathfrak{u}_{*}\right) \in \mathfrak{P}^{\epsilon}$ (see 4.1) and $A$ is a perverse sheaf in $\mathcal{Q}\left(\mathfrak{l}_{\eta}\right)$ which is $\eta$-semicuspidal (as in 1.8 with $H$ replaced by $L$ ).

8.2. An object $B \in \mathcal{Q}\left(\mathfrak{g}_{\delta}\right)$ is said to be $\eta$-quasi-monomial if $B \cong \widetilde{\operatorname{Ind}}_{\mathfrak{p}_{\eta}}^{\mathfrak{g}_{\delta}}(A)$ for some $\left(\mathfrak{p}_{*}, L, P_{0}, \mathfrak{l}, \mathfrak{l}_{*}, \mathfrak{u}_{*}, A\right) \in \mathfrak{R}^{\epsilon}$; if in addition $A$ is taken to be cuspidal, then $B$ is said to be $\eta$-monomial. Using $1.8(\mathrm{~b})$ and the transitivity property 4.2 , we see that:

(a) If $B \in \mathcal{Q}\left(\mathfrak{g}_{\delta}\right)$ is $\eta$-quasi-monomial, then there exists an $\eta$-monomial object $B^{\prime} \in \mathcal{Q}\left(\mathfrak{g}_{\delta}\right)$ such that $B^{\prime} \cong B\left[a_{1}\right] \oplus B\left[a_{2}\right] \oplus \cdots \oplus B\left[a_{k}\right]$ for some sequence $a_{1}, a_{2}, \ldots, a_{k}$ in $\mathbf{Z}, k \geq 1$. In particular, in $\mathcal{K}\left(\mathfrak{g}_{\delta}\right)$ we have $\left(B^{\prime}\right)=\left(v^{a_{1}}+\cdots+\right.$ $\left.v^{a_{k}}\right)(B)$.

An object of $\mathcal{Q}\left(\mathfrak{g}_{\delta}\right)$ is said to be $\eta$-good if it is a direct sum of shifts of $\eta$-quasimonomial objects.

Proposition 8.3 (8.3). Let $B \in \mathcal{Q}\left(\mathfrak{g}_{\delta}\right)$. There exists $\eta$-good objects $B_{1}, B_{2}$ in $\mathcal{Q}\left(\mathfrak{g}_{\delta}\right)$ such that $B \oplus B_{1} \cong B_{2}$.

We can assume that $B$ is a simple perverse sheaf. We define $(\mathcal{O}, \mathcal{L}) \in \mathcal{I}\left(\mathfrak{g}_{\delta}\right)$ by the requirement that $\operatorname{supp} B$ is the closure $\overline{\mathcal{O}}$ of $\mathcal{O}$ in $\mathfrak{g}_{\delta}$ and $\left.B\right|_{\mathcal{O}}=\mathcal{L}[\operatorname{dim} \mathcal{O}]$. We prove the proposition by induction on $\operatorname{dim} \mathcal{O}$. Let $x \in \mathcal{O}$. We associate to $x$ an $\epsilon$-spiral $\mathfrak{p}_{*}=\mathfrak{p}_{*}^{x}$ as in 2.9 ; we complete it uniquely to a system $\left(\mathfrak{p}_{*}, L, P_{0}, \mathfrak{l}, \mathfrak{l}_{*}, \mathfrak{u}_{*}\right) \in \mathfrak{P}^{\epsilon}$. By 7.1(c), there exists $A_{1} \in \mathcal{Q}\left(\mathfrak{l}_{\eta}\right)$ such that ${ }^{\epsilon} \operatorname{Ind}_{\mathfrak{p}_{\eta}} \mathfrak{g}\left(A_{1}\right) \cong B[d] \oplus B^{\prime}$, where $d \in \mathbf{Z}$ and $B^{\prime} \in \mathcal{Q}\left(\mathfrak{g}_{\delta}\right)$ has support contained in $\overline{\mathcal{O}}-\mathcal{O}$. We now use 1.9 (a) for $L, A_{1}$ instead of $H, A_{1}$; applying ${ }^{\epsilon} \operatorname{Ind}_{\mathfrak{p}_{\eta}}^{\mathfrak{g}_{\delta}}$ to the equality in 1.9(a) we obtain

$$
{ }^{\epsilon} \operatorname{Ind}_{\mathfrak{p}_{\eta}}^{\mathfrak{g}_{\delta}}\left(A_{1}\right) \oplus C_{1}^{\prime} \oplus C_{2}^{\prime} \oplus \ldots \oplus C_{t}^{\prime}=C_{t+1}^{\prime} \oplus \ldots \oplus C_{t+t^{\prime}}^{\prime},
$$

where each $C_{j}^{\prime}$ is an $\eta$-quasi-monomial object with a shift (we have used the transitivity property 4.2 ). Thus we have

$$
B[d] \oplus B^{\prime} \oplus C_{1}^{\prime} \oplus C_{2}^{\prime} \oplus \ldots \oplus C_{t}^{\prime}=C_{t+1}^{\prime} \oplus \ldots \oplus C_{t+t^{\prime}}^{\prime}
$$

Now the induction hypothesis implies that $B^{\prime}$ is $\eta$-good. From this and the previous equality we see that $B$ is $\eta$-good. The proposition is proved.

\section{Corollary 8.4.}

(a) The $\mathcal{A}$-module $\mathcal{K}\left(\mathfrak{g}_{\delta}\right)$ is generated by the classes of $\eta$-quasi-monomial objects of $\mathcal{Q}\left(\mathfrak{g}_{\delta}\right)$.

(b) The $\mathbf{Q}(v)$-vector space $\mathbf{Q}(v) \otimes_{\mathcal{A}} \mathcal{K}\left(\mathfrak{g}_{\delta}\right)$ is generated by the classes of $\eta$ monomial objects of $\mathcal{Q}\left(\mathfrak{g}_{\delta}\right)$. 
(a) follows immediately from 8.3; (b) follows from (a) using 8.2(a).

8.5. We show:

(a) If $B_{1}, B_{2}$ are elements of $\mathcal{K}\left(\mathfrak{g}_{\delta}\right)$ then $\left\{B_{1}, B_{2}\right\} \in \mathbf{Q}(v)$ (notation of $4.4(\mathrm{c})$ ).

By 8.3, we can assume that $B_{1}, B_{2}$ are classes of $\eta$-quasi-monomial objects. By 8.2 (a) we have $f_{1} B_{1}=B_{1}^{\prime}, f_{2} B_{2}=B_{2}^{\prime}$ where $B_{1}^{\prime}, B_{2}^{\prime}$ represent $\epsilon$-monomial objects and $f_{1}, f_{2}$ are nonzero elements of $\mathcal{A}$. Thus, we can assume that $B_{1}, B_{2}$ represent $\eta$-monomial objects. In this case the result follows from 6.4 .

\section{ExAmples}

In this section we consider examples where $G=S L(V)$ or $S p(V)$. We assume that $m \geq 2$ and $\eta=1$ hence $\delta=\underline{1}$. We write "spiral" instead of "1-spiral". We explicitly describe the spirals and the set of blocks $\underline{\mathfrak{T}}_{1}$ in both cases, and describe the map $\Psi$ in the case $G=S L(V)$.

9.1. Spirals for the cyclic quiver. We preserve the notation from 0.3 . Thus we assume that $G=S L(V)$ where $V=\oplus_{i \in \mathbf{Z}} / m V_{i}$. We have an induced $\mathbf{Z} / m$-grading on $\mathfrak{g}=\mathfrak{s l}(V)$, so that $\mathfrak{g}_{1}$ is the space of all maps in 0.3(a). In general, we have $\mathfrak{g}_{i}=\oplus_{j \in \mathbf{Z} / m} \operatorname{Hom}\left(V_{j}, V_{j+i}\right)$.

The datum $\lambda \in Y_{G_{0}, \mathbf{Q}}$ is the same as a $\mathbf{Q}$-grading on each $V_{i}$, i.e., $V_{i}=\oplus_{x \in \mathbf{Q}}\left({ }_{x} V_{i}\right)$ such that $\sum_{i} \sum_{x} x \operatorname{dim}\left({ }_{x} V_{i}\right)=0$. Given such a Q-grading on each $V_{i}$, the corresponding spiral $\mathfrak{p}_{*}=\left\{\mathfrak{p}_{N} \subset \mathfrak{g}_{N}\right\}_{N \in \mathbf{Z}}$ takes the following form:

$$
\mathfrak{p}_{N}=\left\{\phi \in \mathfrak{s l}(V) \mid \phi\left({ }_{x} V_{j}\right) \subset \oplus_{x^{\prime} \geq x+N}\left({ }_{x^{\prime}} V_{j+\underline{N}}\right), \quad \forall j \in \mathbf{Z} / m, x \in \mathbf{Q}\right\} .
$$

A splitting $\mathfrak{m}_{*}=\left\{\mathfrak{m}_{N} \subset \mathfrak{g}_{N}\right\}_{N \in \mathbf{Z}}$ of the spiral $\mathfrak{p}_{*}$ takes the form

$$
\mathfrak{m}_{N}=\left\{\phi \in \mathfrak{s l}(V) \mid \phi\left({ }_{x} V_{j}\right) \subset{ }_{x+N} V_{j+\underline{N}}, \quad \forall j \in \mathbf{Z} / m, x \in \mathbf{Q}\right\} .
$$

For such a grading ${ }_{x} V_{i}$ we may introduce a quiver $Q_{\lambda}$ as follows. Let $J_{\lambda}$ be the finite set of pairs $(i, x) \in \mathbf{Z} / m \times \mathbf{Q}$ such that ${ }_{x} V_{i} \neq 0$. Then $Q_{\lambda}$ has vertex set $J_{\lambda}$ and an edge $(i, x) \rightarrow(i+1, x+1)$ if both $(i, x)$ and $(i+1, x+1)$ are in $J_{\lambda}$. Then $Q_{\lambda}$ is a disjoint union of directed chains (that is, quivers of type $A$ with exactly one source and exactly one sink). We may identify $\mathfrak{m}_{1}$ with the representation space of the quiver $Q_{\lambda}$ with vector space ${ }_{x} V_{i}$ on the vertex $(i, x) \in J_{\lambda}$.

Let $B$ be the set of chains in $Q_{\lambda}$, and let $J_{\lambda}=\sqcup_{\beta \in B}\left({ }_{\beta} J_{\lambda}\right)$ be the corresponding decomposition of the vertex set. Let ${ }_{\beta} V:=\oplus_{(i, x) \in \beta}\left({ }_{x} V_{i}\right)$. Then we have $V=\oplus_{\beta \in B}\left({ }_{\beta} V\right)$. Let $M=e^{\mathfrak{m}}, M_{0}=e^{\mathfrak{m}_{0}}$ where $\mathfrak{m}=\oplus_{N} \mathfrak{m}_{N}$. Then $M=S\left(\prod_{\beta \in B} G L\left({ }_{\beta} V\right)\right), M_{0}=S\left(\prod_{(i, x) \in J_{\lambda}} G L\left({ }_{x} V_{i}\right)\right)$. The center $Z_{M}$ is the subgroup of $M$ where each factor in $G L\left({ }_{\beta} V\right)$ is a scalar matrix.

9.2. Admissible systems for the cyclic quiver. Let $d$ be a divisor of $n=\operatorname{dim} V$. Suppose that the following hold:

(1) Each ${ }_{x} V_{i}$ has dimension $\leq 1$.

(2) Each connected component of the quiver $Q_{\lambda}$ is a directed chain containing exactly $d$ vertices.

In this case, $M_{0}$ is a maximal torus of $G$ stabilizing each line ${ }_{x} V_{i}$ for $(i, x) \in J_{\lambda}$. The open $M_{0}$-orbit $\stackrel{\circ}{\mathfrak{m}}_{1} \subset \mathfrak{m}_{1}$ consists of representations of $Q_{\lambda}$ where all arrows are nonzero (hence isomorphisms). The stabilizer of an element in $\stackrel{\circ}{\mathfrak{m}}_{1}$ under $M_{0}$ is exactly $Z_{M}$, which acts by a scalar $z_{\beta}$ on each chain $\beta \in B$, such that $\left(\prod_{\beta \in B} z_{\beta}\right)^{d}=$ 1 . We see that $\pi_{0}\left(Z_{M}\right) \cong \mu_{d}$. For any primitive character $\chi: \mu_{d} \rightarrow \overline{\mathbf{Q}}_{l}^{*}$, we have 
a rank $1 M_{0}$-equivariant local system $C_{\chi}$ on $\stackrel{\circ}{\mathfrak{m}} 1$ on whose stalks $Z_{M}$ acts via $\chi$. This is a cuspidal local system because it is the restriction of the cuspidal local system on the regular nilpotent orbit of $\mathfrak{m}$ with central character $\chi$. Let $\tilde{C}_{\chi}$ be the cuspidal perverse sheaf on $\mathfrak{m}_{1}$ corresponding to $C_{\chi}$. The system $\left(M, M_{0}, \mathfrak{m}, \mathfrak{m}_{*}, \tilde{C}_{\chi}\right)$ is admissible. It is easy to see that any admissible system is of the form we just described.

Given such a grading $\lambda$, we define a function $f: B \rightarrow \mathbf{Z} / m$ such that $f(\beta)=i$ where $(i, x)$ is the head (origin) of the chain $\beta$. Each vertex $(i, x) \in J_{\lambda}$ lies in a unique chain $\beta \in B$ whose head is of the form $\left(f(\beta), x^{\prime}\right)$. Then $x-x^{\prime}=y$ is an integer between 0 and $d-1$ and $f(\beta)+y=i$ in $\mathbf{Z} / m$. This implies that $\operatorname{dim} V_{i}=\sharp\left\{x \in \mathbf{Q} \mid(i, x) \in J_{\lambda}\right\}$ is the same as the number of pairs $(\beta, y) \in B \times$ $\{0,1, \ldots, d-1\}$ such that $f(\beta)+y=i$. Choosing a bijection between $\{1,2, \ldots, n / d\}$ and $B$, the function $f$ may be viewed as a function $\{1,2, \ldots, n / d\} \rightarrow \mathbf{Z} / m$ satisfying 0.7 (b). Changing the bijection amounts to precomposing $f$ with a permutation of $\{1,2, \ldots, n / d\}$. Summarizing the above discussion, we get a canonical bijection between $\underline{\mathfrak{T}}_{1}$ and the set of equivalence classes of triples $(d, f, \chi)$ as in $0.7(\mathrm{a})$.

9.3. The map $\Psi$ for the cyclic quiver. We preserve the notation from 9.1. Let $(\mathcal{O}, \mathcal{L}) \in \chi\left(i \mathfrak{g}_{1}\right)$. For each element $e \in \mathcal{O}$, there exists a decomposition of $V$ into Jordan blocks $\left\{{ }_{\alpha} W\right\}_{\alpha \in B_{e}}$ compatible with the $\mathbf{Z} / m$-grading in the following sense. Each Jordan block ${ }_{\alpha} W$ is a direct sum of finitely many 1-dimensional subspaces indexed by $0,1, \ldots$, i.e., ${ }_{\alpha} W=\left({ }_{\alpha} W_{0}\right) \oplus\left({ }_{\alpha} W_{1}\right) \oplus \ldots$ such that

(1) ${ }_{\alpha} W_{N} \subset V_{h(\alpha)+\underline{N}}$ for some $h(\alpha) \in \mathbf{Z} / m$ (location of the head of the Jordan block $\alpha$ );

(2) $e$ maps ${ }_{\alpha} W_{N}$ isomorphically to ${ }_{\alpha} W_{N+1}$ whenever $N \geq 0$ and ${ }_{\alpha} W_{N+1} \neq 0$.

The datum of $\left\{{ }_{\alpha} W\right\}_{\alpha \in B_{e}}$ as above is the equivalent to the datum of an element $\phi \in J_{1}(e)$; see 2.3. From this we may define a quiver $Q_{e}$ whose vertex set $J_{e}$ consists of pairs $(\alpha, N) \in B_{e} \times \mathbf{Z}_{>0}$ such that ${ }_{\alpha} W_{N} \neq 0$, and there is no edge $(\alpha, N) \rightarrow(\alpha, N+1)$ if both $(\alpha, N),(\alpha, N+1)$ are in $B_{e} \times \mathbf{Z}_{\geq 0}$.

Each vertex $(\alpha, N)$ is labelled with the element $h(\alpha)+N \in \mathbf{Z} / m$. The isomorphism class of $Q_{e}$ together with the labelling by elements in $\mathbf{Z} / m$ is independent of the choice of $e$ in $\mathcal{O}$ and the choice of the Jordan block decomposition. Therefore we denote this labelled quiver by $Q_{\mathcal{O}}$, with vertex set $J_{\mathcal{O}}$ and set of chains $B_{\mathcal{O}}$.

Let $d^{\prime}=\operatorname{gcd}\{|\alpha|\}_{\alpha \in B_{\mathcal{O}}}$ (here $|\alpha|$ is the number of vertices of the chain $\alpha$ ). Then for any $e \in \mathcal{O}$, there is a canonical isomorphism $\pi_{0}\left(G_{0}(e)\right) \cong \mu_{d^{\prime}}$. The local system $\mathcal{L}$ on $\mathcal{O}$ corresponds to a character $\rho$ of $\mu_{d^{\prime}}$, which has order $d$ dividing $d^{\prime}$ and a unique factorization

$$
\rho: \mu_{d^{\prime}} \rightarrow \mu_{d} \stackrel{\chi}{\rightarrow} \overline{\mathbf{Q}}_{l}^{*}
$$

such that $\chi$ is injective (here the first map $\mu_{d^{\prime}} \rightarrow \mu_{d}$ is given by $z \mapsto z^{d^{\prime} / d}$ ). Now we define a new quiver $Q_{\mathcal{O}}^{[d]}$ by removing certain edges from each chain of $Q_{\mathcal{O}}$ such that each chain of $Q_{\mathcal{O}}^{[d]}$ has exactly $d$ vertices. Let $B$ be the set of chains of $Q_{\mathcal{O}}^{[d]}$; then $B$ can be identified with the set $\{1,2, \ldots, n / d\}$. Define $f:\{1,2, \ldots, n / d\} \cong B \rightarrow \mathbf{Z} / m$ to be the map assigning to each $\beta \in B$ the label of its head. This way we get a triple $(d, f, \chi)$ as in $0.7(\mathrm{~b})$ whose equivalence class is well-defined.

Proposition 9.4. In the case of cyclic quivers, the map $\Psi: \mathcal{I}\left(\mathfrak{g}_{1}\right) \rightarrow \underline{\mathfrak{T}}_{1}$ sends $(\mathcal{O}, \mathcal{L})$ to the admissible system in $\underline{\mathfrak{T}}_{1}$ which corresponds to the equivalence class of the triple $(d, f, \chi)$ defined above under the bijection 0.7(a). 
Let $e \in \mathcal{O}$, and let $V=\oplus_{\alpha \in B_{e}}\left({ }_{\alpha} W\right),{ }_{\alpha} W={ }_{\alpha} W_{0} \oplus_{\alpha} W_{1} \oplus \cdots$ be a Jordan block decomposition, where ${ }_{\alpha} W_{N} \subset V_{h(\alpha)+\underline{N}}$ for $\alpha \in B_{e}, N \in \mathbf{Z}_{\geq 0}$. Let $L$ be the Levi subgroup of a parabolic subgroup of $G$ such that $L$ stabilizes the decomposition $V=\oplus_{\alpha \in B_{e}}\left({ }_{\alpha} W\right)$. Then $\mathfrak{l}=\mathfrak{L} L$ has a $\mathbf{Z}$-grading induced from the $\mathbf{Z}$-grading on each of ${ }_{\alpha} W$. In particular, $\mathfrak{l}_{1}$ is the space of representations of the quiver $Q_{e}$. The system $\left(L, L_{0}, \mathfrak{l}, \mathfrak{l}_{*}\right)$ is the system $\left(\tilde{L}^{\phi}, \tilde{L}_{0}^{\phi}, \tilde{\mathfrak{l}}^{\phi}, \tilde{\mathfrak{l}}_{*}^{\phi}\right)$ attached to some $\phi \in J_{\underline{1}}(e)$ as in 2.9. Then $e$ is in the open $L_{0}$-orbit $\mathfrak{l}_{1}$ of $\mathfrak{l}_{1}$, which is contained in the regular nilpotent orbit of $\mathfrak{l}$.

Let ${ }_{\alpha} L=S L\left({ }_{\alpha} W\right)$ be the subgroup of $L$ which acts as identity on all blocks ${ }_{\alpha^{\prime}} W$ for $\alpha^{\prime} \neq \alpha$. Then ${ }_{\alpha} \mathfrak{l}=\mathfrak{L}\left({ }_{\alpha} L\right)$ carries a $\mathbf{Z}$-grading compatible with that on l. For each interval $[a, b] \subset \mathbf{Z}_{\geq 0}$, let ${ }_{\alpha} W_{[a, b]} \subset{ }_{\alpha} W$ be the direct sum of ${ }_{\alpha} W_{N}$ for $a \leq N \leq b$. We decompose ${ }_{\alpha} W$ into $|\alpha| / d$ parts each of dimension $d$ :

$$
{ }_{\alpha} W=\oplus_{j=1}^{|\alpha| / d}\left({ }_{\alpha} W_{[(j-1) d, j d-1]}\right) .
$$

Let ${ }_{\alpha} M \subset{ }_{\alpha} L$ be the subgroup stabilizing the decomposition (a). Then the Lie algebra ${ }_{\alpha} \mathfrak{m}$ of ${ }_{\alpha} M$ inherits a $\mathbf{Z}$-grading from that of ${ }_{\alpha} \mathfrak{l}$, and the open orbit ${ }_{\alpha} \stackrel{\circ}{\mathfrak{m}}_{1}$ carries a local system ${ }_{\alpha} C_{\chi}$ corresponding to the character $\chi$ of $\mu_{d} \cong \pi_{0}\left(Z\left({ }_{\alpha} M\right)\right)$. Let ${ }_{\alpha} \tilde{C}_{\chi}$ be the cuspidal perverse sheaf on ${ }_{\alpha} \mathfrak{m}_{1}$ corresponding to ${ }_{\alpha} C_{\chi}$. Define a parabolic subalgebra ${ }_{\alpha} \mathfrak{q} \subset{ }_{\alpha} \mathfrak{l}$ to be the stabilizer of the filtration ${ }_{\alpha} W_{[|\alpha|-d,|\alpha|-1]} \subset$ ${ }_{\alpha} W_{[|\alpha|-2 d,|\alpha|-1]} \subset \cdots \subset{ }_{\alpha} W={ }_{\alpha} W_{[0,|\alpha|-1]}$. Then ${ }_{\alpha} \mathfrak{q}$ is compatible with the $\mathbf{Z}$ grading on ${ }_{\alpha} \mathfrak{l}$ and ${ }_{\alpha} \mathfrak{m}$ is a Levi subalgebra of ${ }_{\alpha} \mathfrak{q}$. The induction

$$
\operatorname{ind}_{\alpha}^{\alpha \mathfrak{q}_{1}} \mathfrak{l}_{\alpha}\left(\tilde{C}_{\chi}\right)
$$

restricted to ${ }_{\alpha} \mathfrak{l}_{1}$ is isomorphic to $\left.\mathcal{L}\right|_{\alpha} \mathfrak{l}_{1}$, because the map $c$ in 1.3 (applied to ${ }_{\alpha} \mathfrak{l},{ }_{\alpha} \mathfrak{q},{ }_{\alpha} \mathfrak{m}$ in place of $\left.\mathfrak{h}, \mathfrak{p}, \mathfrak{l}\right)$ is an isomorphism when restricted to ${ }_{\alpha} \stackrel{\circ}{\mathfrak{l}}_{1}$. Therefore the middle extension of $\left.\mathcal{L}\right|_{\alpha}{ }_{\alpha}$ to $\mathfrak{l}_{1}$ appears as a direct summand of $\left.\operatorname{ind}_{\alpha}{ }_{\alpha} \mathfrak{q}_{1}{ }_{\alpha}{ }_{\alpha} \tilde{C}_{\chi}\right)$. Therefore, under the map defined in $1.5(\mathrm{~b})$, the image of $\left({ }_{\alpha} \mathfrak{l}_{1},\left.\mathcal{L}\right|_{\alpha}{ }_{\alpha}\right)$ is

$$
\left({ }_{\alpha} M,{ }_{\alpha} M_{0},{ }_{\alpha} \mathfrak{m},{ }_{\alpha} \mathfrak{m}_{1},{ }_{\alpha} \tilde{C}_{\chi}\right) .
$$

Let ${ }_{\alpha} \tilde{M} \subset G L\left({ }_{\alpha} W\right)$ be the stabilizer of the decomposition (a). Let

$$
M=S\left(\prod_{\alpha \in B_{e}}\left({ }_{\alpha} \tilde{M}\right)\right) \subset L
$$

with Lie algebra $\mathfrak{m} \subset \oplus\left({ }_{\alpha} \tilde{\mathfrak{m}}\right)$ and the induced Z-grading from each ${ }_{\alpha} \tilde{\mathfrak{m}}=\mathfrak{L}\left({ }_{\alpha} \tilde{M}\right)$. The open $M_{0}$-orbit on $\mathfrak{m}_{1}=\oplus\left({ }_{\alpha} \mathfrak{m}_{1}\right)$ is $\stackrel{\circ}{\mathfrak{m}}_{1}=\prod\left({ }_{\alpha} \stackrel{\circ}{\mathfrak{m}}_{1}\right)$. Let $C_{\chi}=\otimes\left({ }_{\alpha} C_{\chi}\right)$ on $\stackrel{\circ}{\mathfrak{m}}_{1}$. Let $\tilde{C}_{\chi}$ be the cuspidal perverse sheaf on $\mathfrak{m}_{1}$ corresponding to $C_{\chi}$. By the compatibility of the assignment in $1.5(\mathrm{~b})$ with direct products, in the situation $H=L$, the pair $\left(\stackrel{\circ}{\mathfrak{l}}_{1},\left.\mathcal{L}\right|_{\mathfrak{l}_{1}}\right)$ maps to $\left(M, M_{0}, \mathfrak{m}, \mathfrak{m}_{*}, \tilde{C}_{\chi}\right)$. Therefore, $\left(M, M_{0}, \mathfrak{m}, \mathfrak{m}_{*}, \tilde{C}_{\chi}\right)$ is the admissible system attached to $(\mathcal{O}, \mathcal{L})$ through the procedure in 2.9 . By 9.2 , the admissible system $\left(M, M_{0}, \mathfrak{m}, \mathfrak{m}_{*}, \tilde{C}_{\chi}\right)$ corresponds to the triple $(d, f, \chi)$ defined in 9.3 before the statement of this proposition. This finishes the proof.

9.5. The symplectic quiver. Let $V$ be a finite-dimensional vector space over $\mathbf{k}$ with a nondegenerate symplectic form $\omega$. Assume that $m$ in 0.1 is even. Let $\tilde{\mathfrak{S}}_{m}=\{j ; j=k / 2 ; k=$ an odd integer $\}$ and let $\mathfrak{S}_{m}$ be the set of equivalence 
classes for the relation $\sim$ on $\tilde{\mathfrak{S}}_{m}$ given by $j \sim j^{\prime}$ if $j-j^{\prime} \in m \mathbf{Z}$. Note that the involution $j \mapsto-j$ of $\tilde{\mathfrak{S}}_{m}$ induces an involution of $\mathfrak{S}_{m}$ denoted again by $j \mapsto-j$.

For any $N \in \mathbf{Z}$, the map $j \mapsto N+j$ of $\tilde{\mathfrak{S}}_{m}$ onto itself induces a map of $\mathfrak{S}_{m}$ onto itself which depends only on $\underline{N}$ and is denoted by $j \mapsto \underline{N}+j$.

The set $\mathfrak{S}_{m}$ consists of $m$ elements represented by

$$
\left\{\frac{1}{2}, \frac{3}{2}, \ldots, \frac{m-1}{2}, \frac{m+1}{2}, \ldots, m-\frac{1}{2}\right\} .
$$

Consider a grading on $V$ indexed by $\mathfrak{S}_{m}$ :

$$
V=\bigoplus_{j \in \mathfrak{S}_{m}} V_{j}
$$

such that $\omega\left(V_{j}, V_{j^{\prime}}\right)=0$ unless $j^{\prime}=-j$ (as elements of $\mathfrak{S}_{m}$ ). Using the symplectic form, for $j \in \mathfrak{S}_{m}$ we may identify $V_{j}$ with the dual of $V_{-j}$.

We assume that $G=S p(V)$ and that the $\mathbf{Z} / m$-grading of $\mathfrak{g}=\mathfrak{s p}(V)$ is given by

$$
\mathfrak{g}_{i}=\left\{\phi \in \mathfrak{s p}(V) \mid \phi\left(V_{j}\right) \subset V_{i+j}, \quad \forall j \in \mathfrak{S}_{m}\right\}, \quad \forall i \in \mathbf{Z} / m .
$$

In particular, an element $\phi \in \mathfrak{g}_{\underline{1}}$ is a collection of maps $\phi_{i}: V_{i-\frac{1}{2}} \rightarrow V_{i+\frac{1}{2}}, i \in \mathbf{Z} / m$, which can be represented by a cyclic quiver

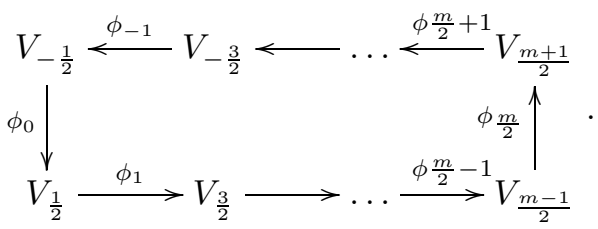

The condition $\phi \in \mathfrak{s p}(V)$ becomes that

$$
\phi_{-i}=-\phi_{i}^{*}, \quad \forall i \in \mathbf{Z} / m .
$$

Here $\phi_{i}^{*}: V_{i+\frac{1}{2}}^{*} \rightarrow V_{i-\frac{1}{2}}^{*}$ is the adjoint of $\phi_{i}$, which can be viewed as a map $V_{-i-\frac{1}{2}} \rightarrow$ $V_{-i+\frac{1}{2}}$ under the identifications $V_{i+\frac{1}{2}}^{*} \cong V_{-i-\frac{1}{2}}, V_{i-\frac{1}{2}}^{*} \cong V_{-i+\frac{1}{2}}$ using the symplectic pairing. In particular, for $i=0, \phi_{0}: V_{-\frac{1}{2}}=V_{\frac{1}{2}}^{*} \rightarrow V_{\frac{1}{2}}$ can be viewed as a vector $\phi_{0} \in V_{\frac{1}{2}}^{\otimes 2}$. The condition (b) for $i=0$ is equivalent to saying that $\phi_{0} \in \operatorname{Sym}^{2}\left(V_{\frac{1}{2}}\right)$. Similarly, we may view $\phi_{\frac{m}{2}}$ as a vector in $V_{\frac{m+1}{2}}^{\otimes 2}$, and the condition (c) for $i=\frac{m}{2}$ is equivalent to saying that $\phi_{\frac{m}{2}} \in \operatorname{Sym}^{2}\left(V_{\frac{m+1}{2}}\right)$.

We call a representation of the quiver above in which $V_{-j}=V_{j}^{*}$, and (c) holds a symplectic representation. In other words, $\mathfrak{g}_{1}$ is the space of symplectic representations of the quiver above.

We have $G_{\underline{0}} \cong \prod_{\frac{1}{2} \leq j \leq \frac{m-1}{2}} G L\left(V_{j}\right)$, where $G L\left(V_{j}\right) \cong G L\left(V_{-j}\right)$ acts diagonally on both $V_{j}$ and $\bar{V}_{-j}=V_{m-j}=V_{j}^{*}$.

9.6. Spirals for the symplectic quiver. Each element $\lambda \in Y_{G_{0}, \mathbf{Q}}$ is the same datum as a Q-grading on each $V_{j}, j \in \mathfrak{S}_{m}$, i.e., $V_{j}=\oplus_{x \in \mathbf{Q}}\left({ }_{x} V_{j}\right)$ such that under the symplectic form $\omega, \omega\left({ }_{x} V_{j}, x^{\prime} V_{-j}\right)=0$ unless $x+x^{\prime}=0$. Then ${ }_{-x} V_{-j}$ can be identified with the dual of ${ }_{x} V_{j}$ for all $(j, x) \in \mathfrak{S}_{m} \times \mathbf{Q}$. The spiral $\mathfrak{p}_{*}$ associated to this grading is

$$
\mathfrak{p}_{N}=\left\{\phi \in \mathfrak{s p}(V) \mid \phi\left({ }_{x} V_{j}\right) \subset \oplus_{x^{\prime} \geq x+N}\left({ }_{x^{\prime}} V_{j+\underline{N}}\right), \quad \forall j \in \mathfrak{S}_{m}, x \in \mathbf{Q}\right\} .
$$


A splitting $\mathfrak{m}_{*}$ of the spiral $\mathfrak{p}_{*}$ takes the form

$$
\mathfrak{m}_{N}=\left\{\pi \in \mathfrak{s p}(V) \mid \phi\left({ }_{x} V_{j}\right) \subset{ }_{x+N} V_{j+\underline{N}}, \quad \forall j \in \mathfrak{S}_{m}, x \in \mathbf{Q}\right\} .
$$

To each such grading, we may attach a quiver $Q_{\lambda}$ as we did for the cyclic quiver (since the symplectic quiver is a special case of a cyclic quiver). There is an involution on $Q_{\lambda}$ sending $(j, x) \in J_{\lambda}$ to $(-j,-x) \in J_{\lambda}$. This involution stabilizes at most two chains $Q_{\lambda}^{\prime}$ and $Q_{\lambda}^{\prime \prime}$ of $Q_{\lambda}$. The set of vertices of $Q_{\lambda}^{\prime}$ (possibly empty) is $J_{\lambda}^{\prime}:=\left\{\left.(x, x)\right|_{x} V_{x} \neq 0\right\} \subset J_{\lambda}$. The set of vertices of $Q_{\lambda}^{\prime \prime}$ (possibly empty) is $J_{\lambda}^{\prime \prime}:=\left\{\left.\left(x-\frac{m}{2}, x\right)\right|_{x} V_{x-\frac{m}{2}} \neq 0\right\} \subset J_{\lambda}$.

9.7. Admissible systems for the symplectic quiver. Suppose that the following hold:

(1) For each $(j, x) \in J-\left(J_{\lambda}^{\prime} \sqcup J_{\lambda}^{\prime \prime}\right)$, we have $\operatorname{dim}_{x} V_{j}=1$.

(2) The chains in $Q_{\lambda}$ other than $Q_{\lambda}^{\prime}$ and $Q_{\lambda}^{\prime \prime}$ all consist of a single vertex.

(3) Let $\sharp J_{\lambda}^{\prime}=2 a^{\prime}$ for some $a^{\prime} \in \mathbf{Z}_{\geq 0}$. When $a^{\prime}>0,\left(-a^{\prime}+\frac{1}{2},-a^{\prime}+\frac{1}{2}\right)$ is the head of $J_{\lambda}^{\prime}$ and $\left(a^{\prime}-\frac{1}{2}, a^{\prime}-\frac{1}{2}\right)$ is the tail. Then $\operatorname{dim}_{x} V_{x}=a^{\prime}+\frac{1}{2}-|x|$ for all $(x, x) \in J_{\lambda}^{\prime}$.

(4) Let $\sharp J_{\lambda}^{\prime \prime}=2 a^{\prime \prime}$ for some $a^{\prime \prime} \in \mathbf{Z}_{\geq 0}$. When $a^{\prime \prime}>0,\left(-a^{\prime \prime}-\frac{m-1}{2},-a^{\prime \prime}+\frac{1}{2}\right)$ is the head of $J_{\lambda}^{\prime \prime}$ and $\left(a^{\prime \prime}-\frac{m+1}{2}, a^{\prime \prime}-\frac{1}{2}\right)$ is the tail. Then $\operatorname{dim}_{x} V_{x-\frac{m}{2}}=a^{\prime \prime}+\frac{1}{2}-|x|$ for all $\left(x-\frac{m}{2}, x\right) \in J_{\lambda}^{\prime \prime}$.

Under these assumptions, $\mathfrak{m}_{1}=\mathfrak{m}_{1}^{\prime} \oplus \mathfrak{m}_{1}^{\prime \prime}$, where $\mathfrak{m}_{1}^{\prime}$ is the space of representations of the quiver $Q_{\lambda}^{\prime}$ with dimension vector $\operatorname{dim}_{x} V_{x}=a^{\prime}+\frac{1}{2}-|x|$ and satisfying the duality condition $\psi_{i}=-\psi_{-i}^{*}$ (where $\psi_{i}:{ }_{i-\frac{1}{2}} V_{i-\frac{1}{2}} \rightarrow_{i+\frac{1}{2}} V_{i+\frac{1}{2}}$ ) for all $i \in$ $\left\{-a^{\prime}+1, \ldots, a^{\prime}-1\right\}$. Similarly, $\mathfrak{m}_{1}^{\prime \prime}$ is the space of representations of the quiver $Q_{\lambda}^{\prime \prime}$ with dimension vector $\operatorname{dim}_{x} V_{x-\frac{m}{2}}=a^{\prime \prime}+\frac{1}{2}-|x|$ and satisfying the duality condition $\psi_{i}=-\psi_{-i}^{*}$. The open $M_{0}$-orbit $\stackrel{\circ}{\mathfrak{m}}_{1}$ consists of those representations of $Q_{\lambda}^{\prime}$ and $Q_{\lambda}^{\prime \prime}$ where each arrow has maximal rank (either injective or surjective).

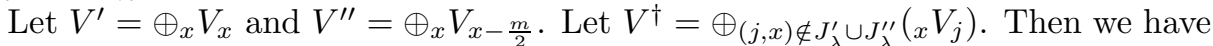
$V=V^{\prime} \oplus V^{\prime \prime} \oplus V^{\dagger}$. This decomposition is preserved by $M$, and $M \cong S p\left(V^{\prime}\right) \times$ $S p\left(V^{\prime \prime}\right) \times T^{\dagger}$, where $T^{\dagger}$ is the maximal torus in $S p\left(V^{\dagger}\right)$ stabilizing each line ${ }_{x} V_{j} \subset$ $V^{\dagger}$. The center $Z_{M}$ is isomorphic to $\{ \pm 1\} \times\{ \pm 1\} \times T^{\dagger}$ under this decomposition. The stabilizer of a point in $\stackrel{\circ}{\mathfrak{m}}_{1}$ under $M_{0}$ is exactly $Z_{M}$. Let $C$ be the rank one local system on $\stackrel{\circ}{\mathfrak{m}}_{1}$ on whose stalks $\pi_{0}\left(Z_{M}\right)$ acts nontrivially on both factors of $\{ \pm 1\}$. Then $C$ is cuspidal because it is the restriction of the unique cuspidal local system on $\mathfrak{m}$. Let $\tilde{C}$ be the cuspidal perverse sheaf on $\mathfrak{m}_{1}$ defined by $C$. The system $\left(M, M_{0}, \mathfrak{m}, \mathfrak{m}_{*}, \tilde{C}\right)$ is admissible. Moreover, any admissible system is of this form. Under $G_{0}$-conjugacy, the only invariant of an admissible system is given by the numbers $a^{\prime}$ and $a^{\prime \prime}$. Since $\operatorname{dim} V_{j}^{\prime}+\operatorname{dim} V_{j}^{\prime \prime} \leq \operatorname{dim} V_{j}$, we have the following inequality for all $j \in \mathfrak{S}_{m}$ :

$$
\begin{aligned}
& \operatorname{dim} V_{j} \geq \sharp\left\{-a^{\prime}+\frac{1}{2} \leq x \leq a^{\prime}-\frac{1}{2} \mid x \equiv j \bmod m \mathbf{Z}\right\} \\
& +\sharp\left\{-a^{\prime \prime}+\frac{1}{2} \leq x \leq a^{\prime \prime}-\frac{1}{2} \mid x \equiv j+\frac{m}{2} \quad \bmod m \mathbf{Z}\right\} .
\end{aligned}
$$

To summarize, we have a natural bijection

$$
\underline{\mathfrak{I}}_{1} \leftrightarrow\left\{\left(a^{\prime}, a^{\prime \prime}\right) \in \mathbf{Z}_{\geq 0} \times \mathbf{Z}_{\geq 0} \text { satisfying (a) for all } j \in \mathfrak{S}_{m}\right\} .
$$

The map $\Psi: \mathcal{I}\left(\mathfrak{g}_{1}\right) \rightarrow \underline{\mathfrak{T}}_{1}$ for the symplectic quiver as well as other graded Lie algebras of classical type will be described in a sequel to this paper using the combinatorics of symbols. 


\section{REFERENCES}

[KL] David Kazhdan and George Lusztig, Proof of the Deligne-Langlands conjecture for Hecke algebras, Invent. Math. 87 (1987), no. 1, 153-215, DOI 10.1007/BF01389157. MR862716

[Ko] Bertram Kostant, The principal three-dimensional subgroup and the Betti numbers of a complex simple Lie group, Amer. J. Math. 81 (1959), 973-1032, DOI 10.2307/2372999. MR0114875

[L1] G. Lusztig, Intersection cohomology complexes on a reductive group, Invent. Math. $\mathbf{7 5}$ (1984), no. 2, 205-272, DOI 10.1007/BF01388564. MR732546

[L2] George Lusztig, Character sheaves. I, Adv. in Math. 56 (1985), no. 3, 193-237, DOI 10.1016/0001-8708(85)90034-9. MR792706

[L3] George Lusztig, Character sheaves. II, Adv. in Math. 57 (1985), no. 3, 266-315, DOI 10.1016/0001-8708(85)90064-7. MR 806210

[L4] George Lusztig, Study of perverse sheaves arising from graded Lie algebras, Adv. Math. 112 (1995), no. 2, 147-217, DOI 10.1006/aima.1995.1031. MR1327095

[RR] L. Rider and A. Russell, Perverse sheaves on the nilpotent cone and Lusztig's generalized Springer correspondence, arxiv:1409.7132.

[L5] G. Lusztig, Study of antiorbital complexes, Representation theory and mathematical physics, Contemp. Math., vol. 557, Amer. Math. Soc., Providence, RI, 2011, pp. 259-287, DOI $10.1090 / \mathrm{conm} / 557 / 11036$. MR 2848930

[St] Robert Steinberg, Endomorphisms of linear algebraic groups, Memoirs of the American Mathematical Society, No. 80, American Mathematical Society, Providence, R.I., 1968. MR0230728

[Vi] Ł̀. B. Vinberg, The Weyl group of a graded Lie algebra (Russian), Izv. Akad. Nauk SSSR Ser. Mat. 40 (1976), no. 3, 488-526, 709. MR0430168

Department of Mathematics, Massachusetts institute of Technology, Cambridge, MASSSACHUSETTS 02139

E-mail address: gyuri@math.mit.edu

Department of Mathematics, Yale University, New Haven, Connecticut 06511

E-mail address: zhiweiyun@gmail.com 